Supporting Information for

\title{
Total Synthesis of Olivacine and Ellipticine via a Lactone Ring-Opening and Aromatization Cascade
}

\author{
Ömer Dilek, ${ }^{\dagger} \ddagger$ Süleyman Patir, ${ }^{\dagger}$ Tahir Tilki, ${ }^{\ddagger}$ Erkan Ertürk* ${ }^{*}$ \\ † Institute of Chemical Technology, TÜBITAK Marmara Research Center, 41470 Gebze, Kocaeli, Turkey \\ ‡ Department of Chemistry, Süleyman Demirel University, 32260 Isparta, Turkey \\ E-mail: erkan.erturk@tubitak.gov.tr
}

\section{Table of Contents}

\begin{tabular}{|c|c|}
\hline Page(s) & Content \\
\hline S1-S3 & Table of Contents \\
\hline S4 & ${ }^{1} \mathrm{H}-\mathrm{NMR}$ Spectrum of $\mathbf{2 a}$ \\
\hline S5 & ${ }^{13} \mathrm{C}-\mathrm{NMR}$ Spectrum of $\mathbf{2 a}$ \\
\hline S6 & ${ }^{1} \mathrm{H}-\mathrm{NMR}$ Spectrum of $\mathbf{3 a}$ \\
\hline S7 & ${ }^{13} \mathrm{C}-\mathrm{NMR}$ Spectrum of $\mathbf{3 a}$ \\
\hline S8 & ${ }^{1} \mathrm{H}-\mathrm{NMR}$ Spectrum of $\mathbf{5 a}$ \\
\hline S9 & ${ }^{13} \mathrm{C}-\mathrm{NMR}$ Spectrum of $\mathbf{5 a}$ \\
\hline S10 & ${ }^{1} \mathrm{H}-\mathrm{NMR}$ Spectrum of $\mathbf{7 a}$ \\
\hline S11 & ${ }^{13} \mathrm{C}-\mathrm{NMR}$ Spectrum of $\mathbf{7 a}$ \\
\hline S12 & ${ }^{1} \mathrm{H}-\mathrm{NMR}$ Spectrum of $\mathbf{9 a}$ \\
\hline S13 & ${ }^{13} \mathrm{C}-\mathrm{NMR}$ Spectrum of $\mathbf{9 a}$ \\
\hline S14 & ${ }^{1} \mathrm{H}-\mathrm{NMR}$ Spectrum of $\mathbf{9 a}$ \\
\hline S15 & ${ }^{13} \mathrm{C}-\mathrm{NMR}$ Spectrum of $\mathbf{9 a}$ \\
\hline S16-18 & HSQC Spectrum of $\mathbf{9 a}$ \\
\hline S19 & NOE-DIFF Spectrum of $\mathbf{9 a}$ \\
\hline $\mathbf{S 2 0}$ & Table S1. NMR spectral data of compound $\mathbf{1 3}$. \\
\hline S21 & ${ }^{1} \mathrm{H}-\mathrm{NMR}$ Spectrum of $\mathbf{1 3}$ \\
\hline S22 & ${ }^{13} \mathrm{C}-\mathrm{NMR}$ Spectrum of $\mathbf{1 3}$ \\
\hline S23-25 & HSQC Spectrum of $\mathbf{1 3}$ \\
\hline S26-28 & COSY Spectrum of $\mathbf{1 3}$ \\
\hline
\end{tabular}




\begin{tabular}{|c|c|}
\hline S29-30 & NOE-DIFF Spectrum of $\mathbf{1 3}$ \\
\hline S31 & ${ }^{1} \mathrm{H}-\mathrm{NMR}$ Spectrum of $\mathbf{1 5 a}$ \\
\hline S32 & ${ }^{13} \mathrm{C}-\mathrm{NMR}$ Spectrum of $\mathbf{1 5 a}$ \\
\hline S33 & ${ }^{1} \mathrm{H}-\mathrm{NMR}$ Spectrum of $\mathbf{1 7}$ \\
\hline S34 & ${ }^{13} \mathrm{C}-\mathrm{NMR}$ Spectrum of $\mathbf{1 7}$ \\
\hline S35 & ${ }^{1} \mathrm{H}-\mathrm{NMR}$ Spectrum of $\mathbf{2 1 a}$ \\
\hline S36 & ${ }^{13} \mathrm{C}-\mathrm{NMR}$ Spectrum of $\mathbf{2 1 a}$ \\
\hline S37 & ${ }^{1} \mathrm{H}-\mathrm{NMR}$ Spectrum of $\mathbf{2 3 a}$ \\
\hline S38 & ${ }^{13} \mathrm{C}-\mathrm{NMR}$ Spectrum of $\mathbf{2 3 a}$ \\
\hline S39 & ${ }^{1} \mathrm{H}-\mathrm{NMR}$ Spectrum of $\mathbf{2 4 a}$ \\
\hline S40 & ${ }^{13} \mathrm{C}-\mathrm{NMR}$ Spectrum of $\mathbf{2 4 a}$ \\
\hline S41 & ${ }^{1} \mathrm{H}-\mathrm{NMR}$ Spectrum of $\mathbf{2 5}$ \\
\hline S42 & ${ }^{13} \mathrm{C}-\mathrm{NMR}$ Spectrum of $\mathbf{2 5}$ \\
\hline S43 & ${ }^{1} \mathrm{H}-\mathrm{NMR}$ Spectrum of $\mathbf{2 6}$ \\
\hline S44 & ${ }^{13} \mathrm{C}-\mathrm{NMR}$ Spectrum of $\mathbf{2 6}$ \\
\hline S45 & ${ }^{1} \mathrm{H}-\mathrm{NMR}$ Spectrum of Olivacine \\
\hline S46 & ${ }^{13} \mathrm{C}-\mathrm{NMR}$ Spectrum of Olivacine \\
\hline S47 & ${ }^{1} \mathrm{H}-\mathrm{NMR}$ Spectrum of $\mathbf{2 b}$ \\
\hline S48 & ${ }^{13} \mathrm{C}-\mathrm{NMR}$ Spectrum of $\mathbf{2 b}$ \\
\hline S49 & ${ }^{1} \mathrm{H}-\mathrm{NMR}$ Spectrum of $\mathbf{3 b}$ \\
\hline S50 & ${ }^{13} \mathrm{C}-\mathrm{NMR}$ Spectrum of $\mathbf{3 b}$ \\
\hline S51 & Table S2. NMR spectral data of compound $\mathbf{5 b}$. \\
\hline S52 & ${ }^{1} \mathrm{H}-\mathrm{NMR}$ Spectrum of $\mathbf{5 b} / \mathbf{6 b}$ \\
\hline $\mathbf{S 5 3}$ & ${ }^{13} \mathrm{C}-\mathrm{NMR}$ Spectrum of $\mathbf{5 b} / \mathbf{6} \mathbf{b}$ \\
\hline S54-56 & HSQC Spectrum of $\mathbf{5 b} / \mathbf{6 b}$ \\
\hline S57-59 & COSY Spectrum of $\mathbf{5 b} / \mathbf{6 b}$ \\
\hline S60-61 & NOE-DIFF Spectrum of $\mathbf{5 b} / \mathbf{6} \mathbf{b}$ \\
\hline S62 & ${ }^{1} \mathrm{H}-\mathrm{NMR}$ Spectrum of $\mathbf{7 b} / \mathbf{8 b}$ \\
\hline S63 & ${ }^{13} \mathrm{C}-\mathrm{NMR}$ Spectrum of $\mathbf{7 b} / \mathbf{8 b}$ \\
\hline S64 & Table S3. NMR spectral data of compound $\mathbf{9 b}$. \\
\hline S65 & ${ }^{1} \mathrm{H}-\mathrm{NMR}$ Spectrum of $\mathbf{9 b}$ \\
\hline S66 & ${ }^{13} \mathrm{C}-\mathrm{NMR}$ Spectrum of $\mathbf{9 b}$ \\
\hline
\end{tabular}




\begin{tabular}{|c|c|}
\hline S67-69 & HSQC Spectrum of $\mathbf{9 b}$ \\
\hline S70-72 & COSY Spectrum of $\mathbf{9 b}$ \\
\hline S73-74 & NOE-DIFF Spectrum of $\mathbf{9 b}$ \\
\hline S75 & Table S4. NMR spectral data of compound $\mathbf{1 0 b}$. \\
\hline S76 & ${ }^{1} \mathrm{H}-\mathrm{NMR}$ Spectrum of $\mathbf{1 0 b}$ \\
\hline S77 & ${ }^{13} \mathrm{C}-\mathrm{NMR}$ Spectrum of $\mathbf{1 0 b}$ \\
\hline S78-80 & HSQC Spectrum of $\mathbf{1 0 b}$ \\
\hline S81-83 & COSY Spectrum of $\mathbf{1 0 b}$ \\
\hline S84-85 & NOE-DIFF Spectrum of $\mathbf{1 0 b}$ \\
\hline S86 & ${ }^{1} \mathrm{H}-\mathrm{NMR}$ Spectrum of $\mathbf{1 5 b}$ \\
\hline S87 & ${ }^{13} \mathrm{C}-\mathrm{NMR}$ Spectrum of $\mathbf{1 5 b}$ \\
\hline S88 & ${ }^{1} \mathrm{H}-\mathrm{NMR}$ Spectrum of $\mathbf{2 1 b}$ \\
\hline S89 & ${ }^{13} \mathrm{C}-\mathrm{NMR}$ Spectrum of $\mathbf{2 1 b}$ \\
\hline S90 & ${ }^{1} \mathrm{H}-\mathrm{NMR}$ Spectrum of $\mathbf{2 3 b}$ \\
\hline S91 & ${ }^{13} \mathrm{C}-\mathrm{NMR}$ Spectrum of $\mathbf{2 3 b}$ \\
\hline S92 & ${ }^{1} \mathrm{H}-\mathrm{NMR}$ Spectrum of $\mathbf{2 4 b}$ \\
\hline S93 & ${ }^{13} \mathrm{C}-\mathrm{NMR}$ Spectrum of $\mathbf{2 4 b}$ \\
\hline S94 & ${ }^{1} \mathrm{H}-\mathrm{NMR}$ Spectrum of 27 \\
\hline S95 & ${ }^{13} \mathrm{C}-\mathrm{NMR}$ Spectrum of $\mathbf{2 7}$ \\
\hline S96 & ${ }^{1} \mathrm{H}-\mathrm{NMR}$ Spectrum of $\mathbf{2 8}$ \\
\hline S97 & ${ }^{13} \mathrm{C}-\mathrm{NMR}$ Spectrum of $\mathbf{2 8}$ \\
\hline S98 & ${ }^{1} \mathrm{H}-\mathrm{NMR}$ Spectrum of Ellipticine \\
\hline S99 & ${ }^{13} \mathrm{C}$-NMR Spectrum of Ellipticine \\
\hline S100 & ${ }^{1} \mathrm{H}-\mathrm{NMR}$ Spectrum of $\mathbf{3 1}$ \\
\hline S101 & ${ }^{13} \mathrm{C}-\mathrm{NMR}$ Spectrum of $\mathbf{3 1}$ \\
\hline $\mathbf{S 1 0 2}$ & ${ }^{1} \mathrm{H}-\mathrm{NMR}$ Spectrum of $\mathbf{3 2}$ \\
\hline $\mathbf{S 1 0 3}$ & ${ }^{13} \mathrm{C}-\mathrm{NMR}$ Spectrum of $\mathbf{3 2}$ \\
\hline S104 & ${ }^{1} \mathrm{H}-\mathrm{NMR}$ Spectrum of $\mathbf{3 5}$ \\
\hline S105 & ${ }^{13} \mathrm{C}-\mathrm{NMR}$ Spectrum of $\mathbf{3 5}$ \\
\hline
\end{tabular}




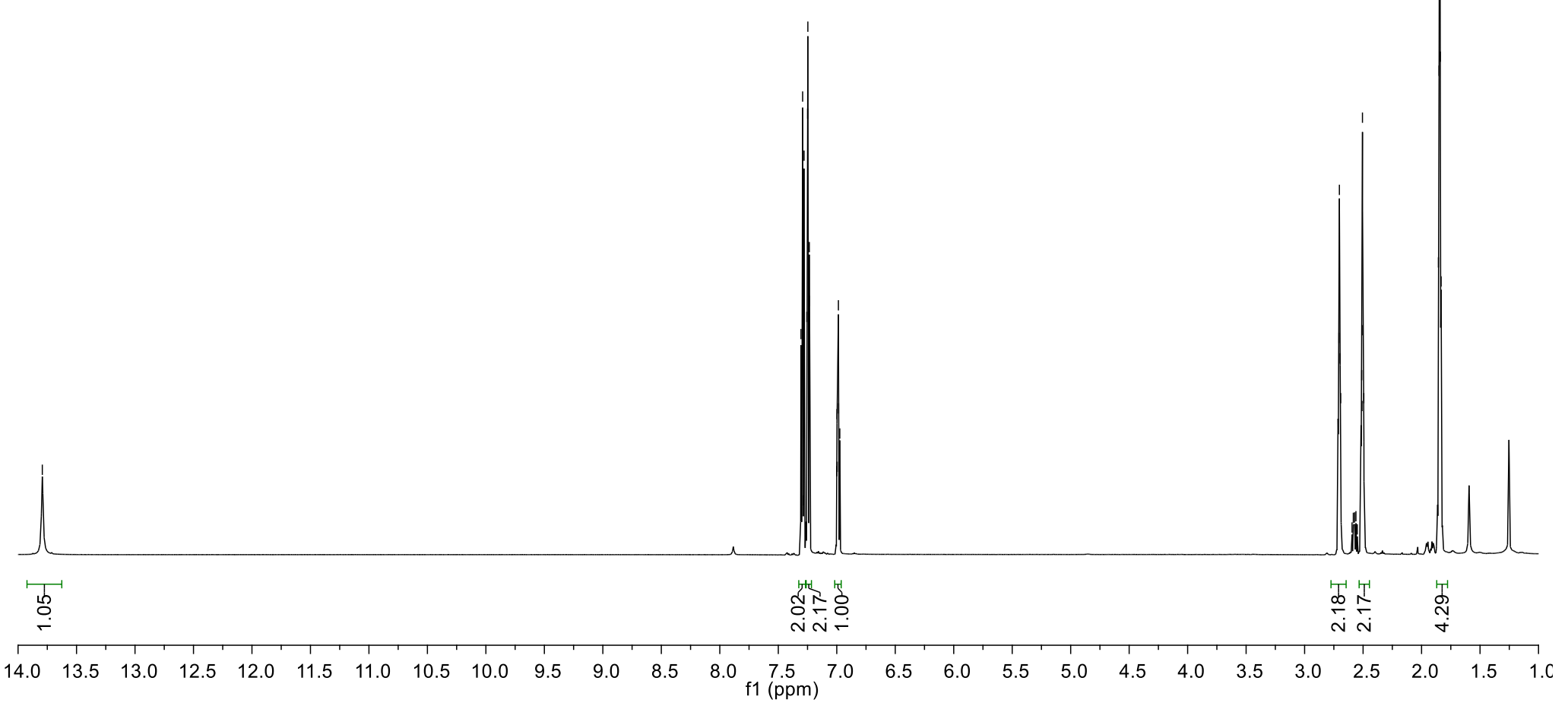

${ }^{1} \mathrm{H}-\mathrm{NMR}$ Spectrum of $2 \mathrm{a}\left(600 \mathrm{MHz}, \mathrm{CDCl}_{3}\right)$ 


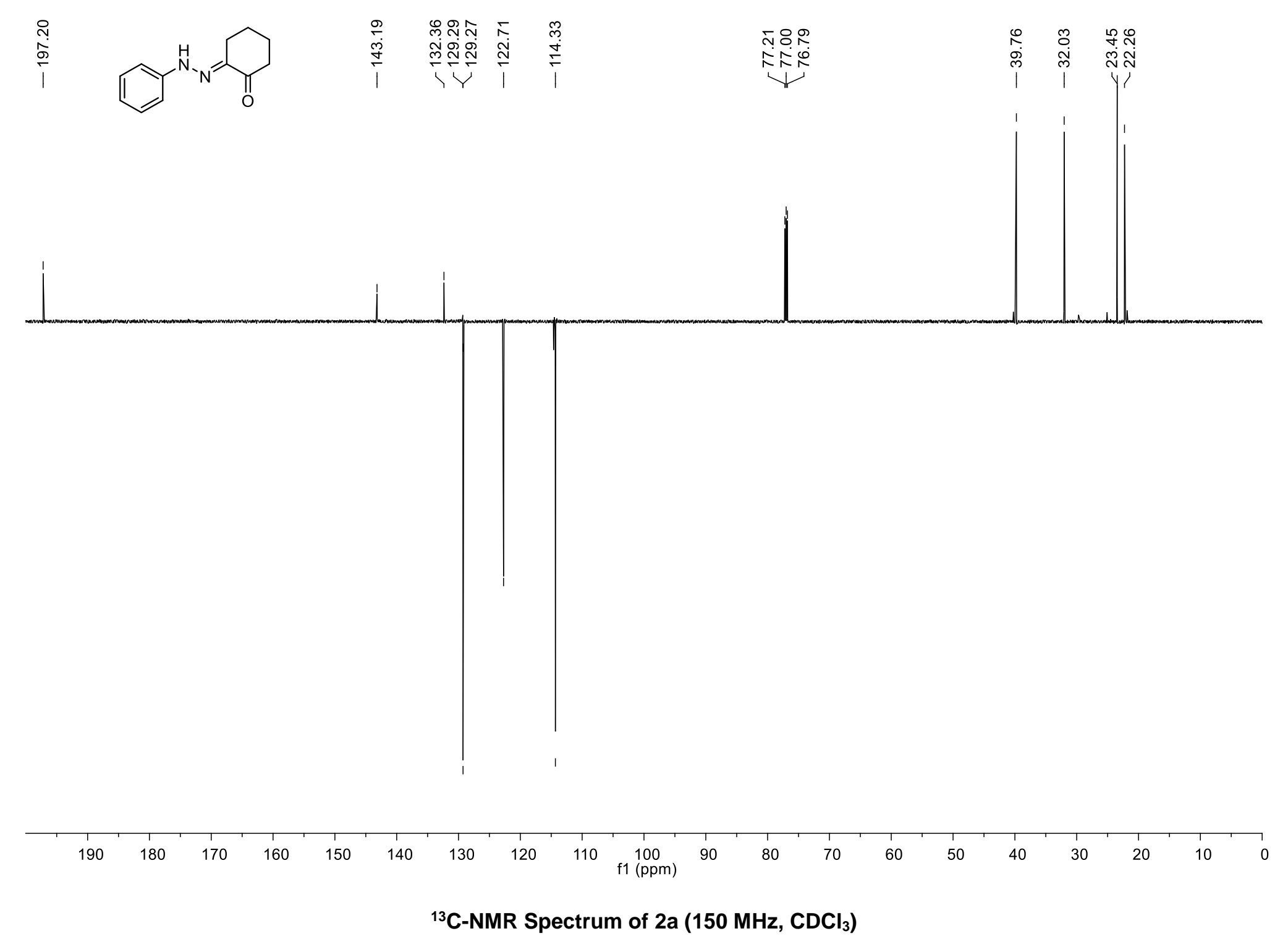




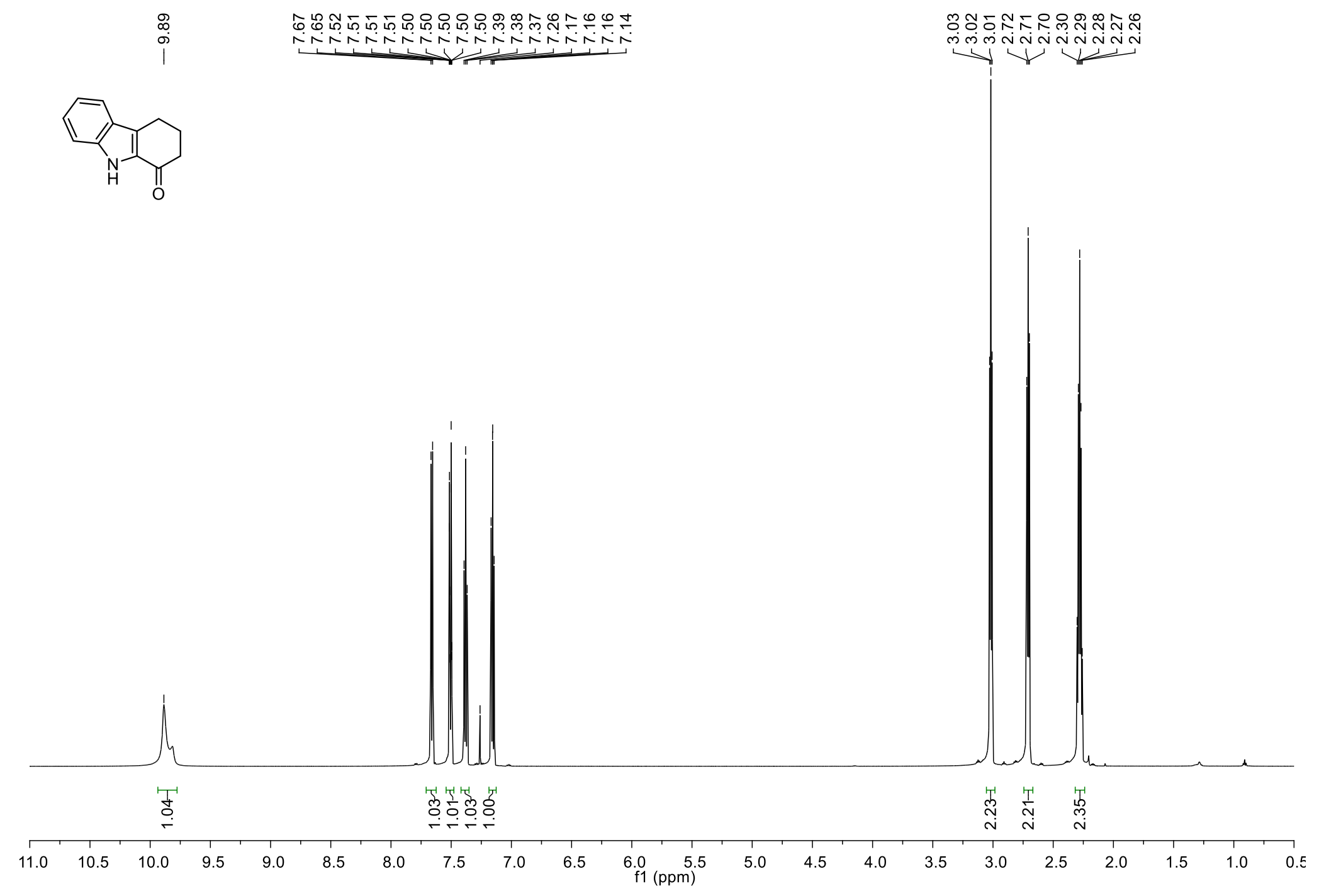

${ }^{1} \mathrm{H}-\mathrm{NMR}$ Spectrum of $3 \mathrm{a}\left(600 \mathrm{MHz}, \mathrm{CDCl}_{3}\right)$ 


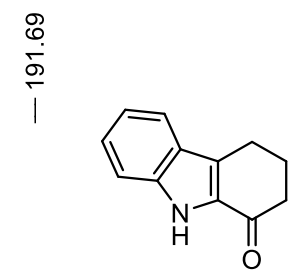

유ำกำก

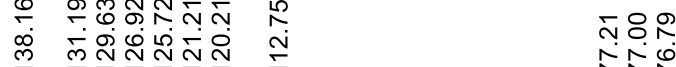

। \行।

상

$\sqrt[4]{2 \pi}$
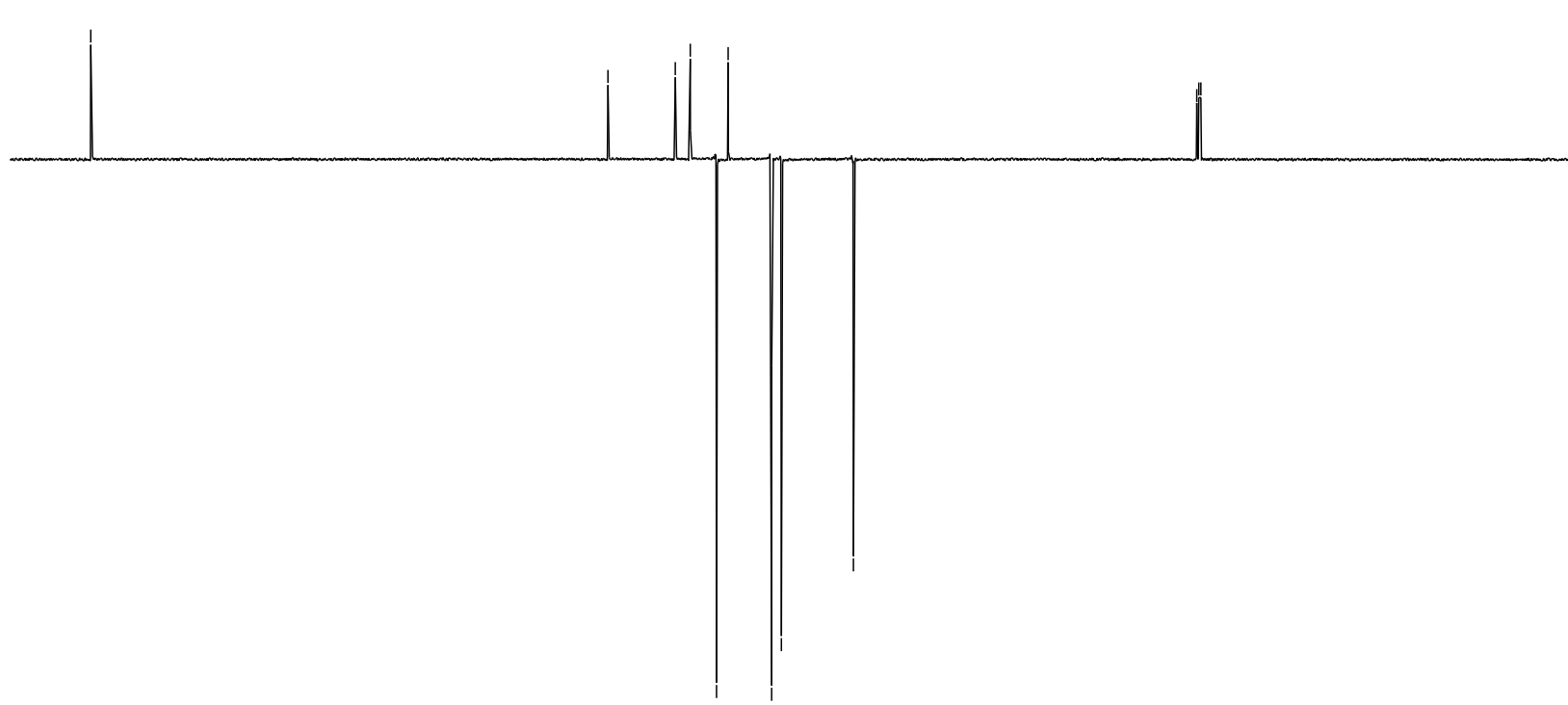

200

$190 \quad 180$

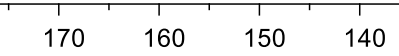

$140 \quad 130$

120

$110 \quad 100$

90

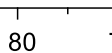

${ }^{13} \mathrm{C}-\mathrm{NMR}$ Spectrum of $3 \mathrm{a}\left(150 \mathrm{MHz}, \mathrm{CDCl}_{3}\right)$ 

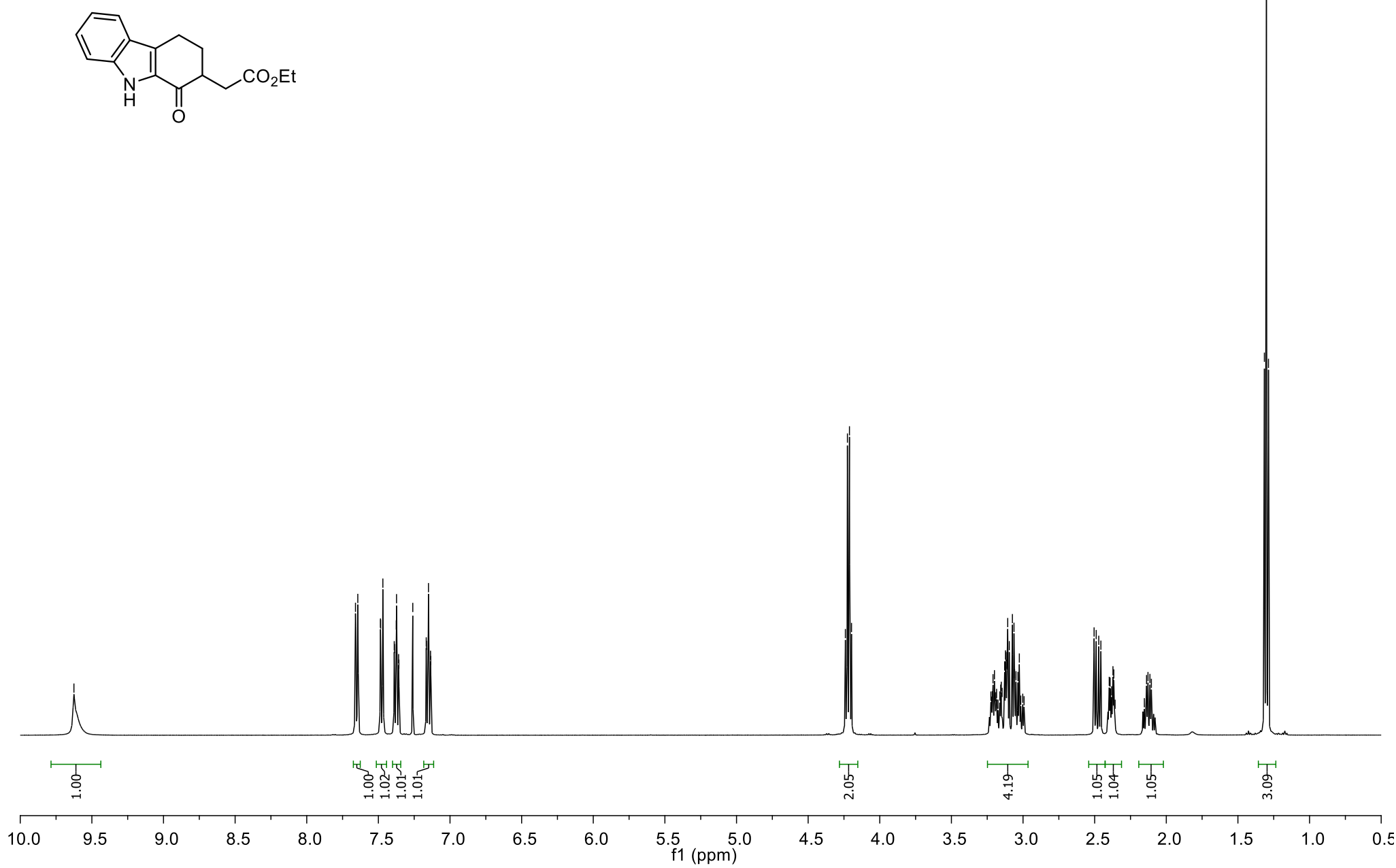

${ }^{1} \mathrm{H}-\mathrm{NMR}$ Spectrum of $5 \mathrm{a}\left(600 \mathrm{MHz}, \mathrm{CDCl}_{3}\right)$ 


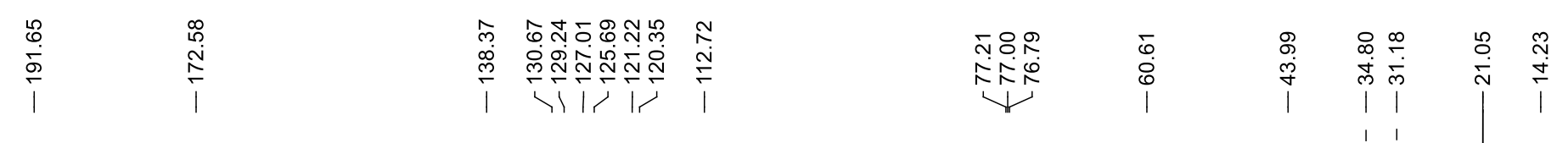
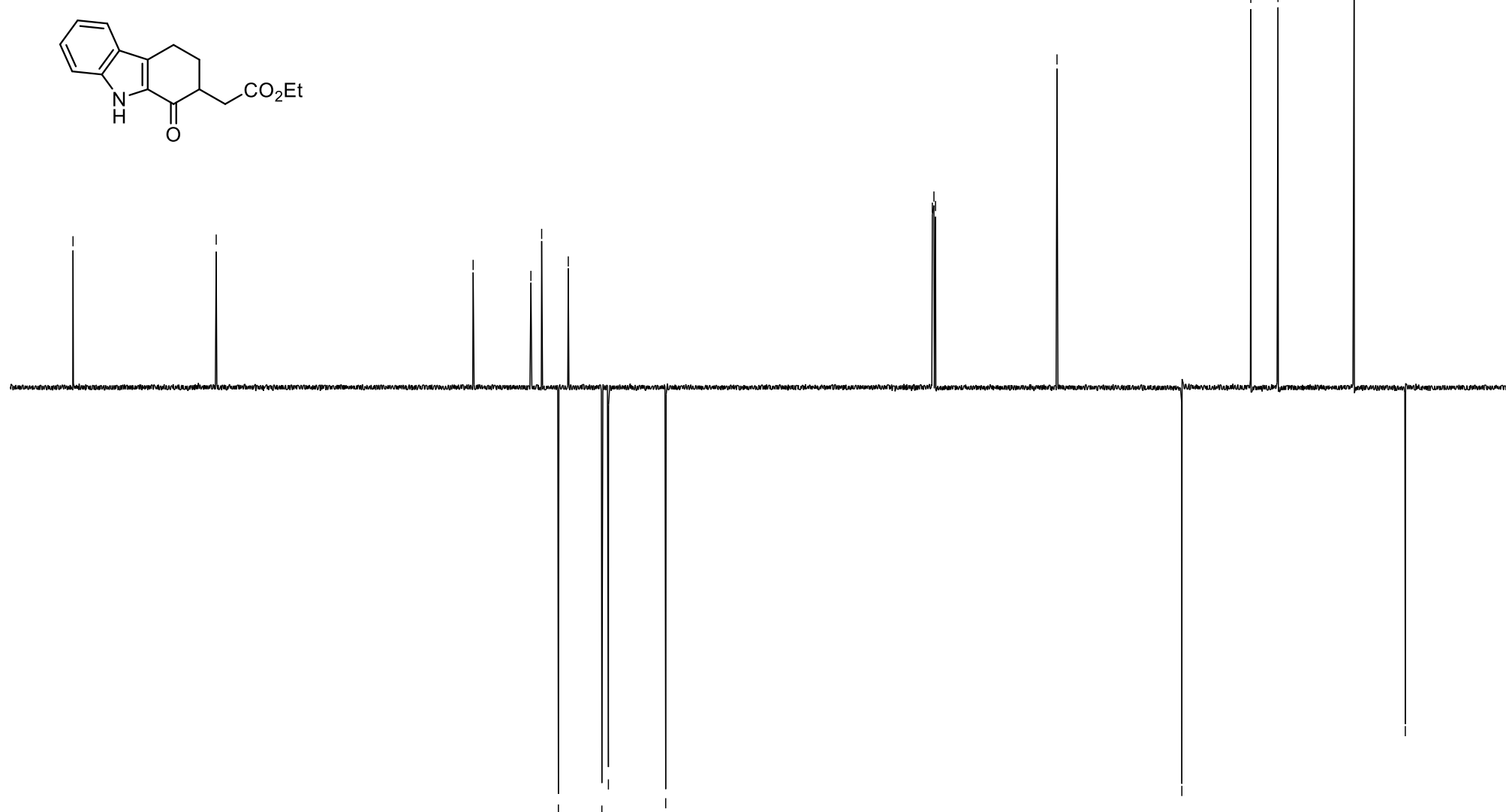

${ }^{13} \mathrm{C}$-NMR Spectrum of $5 \mathrm{a}\left(150 \mathrm{MHz}, \mathrm{CDCl}_{3}\right)$ 


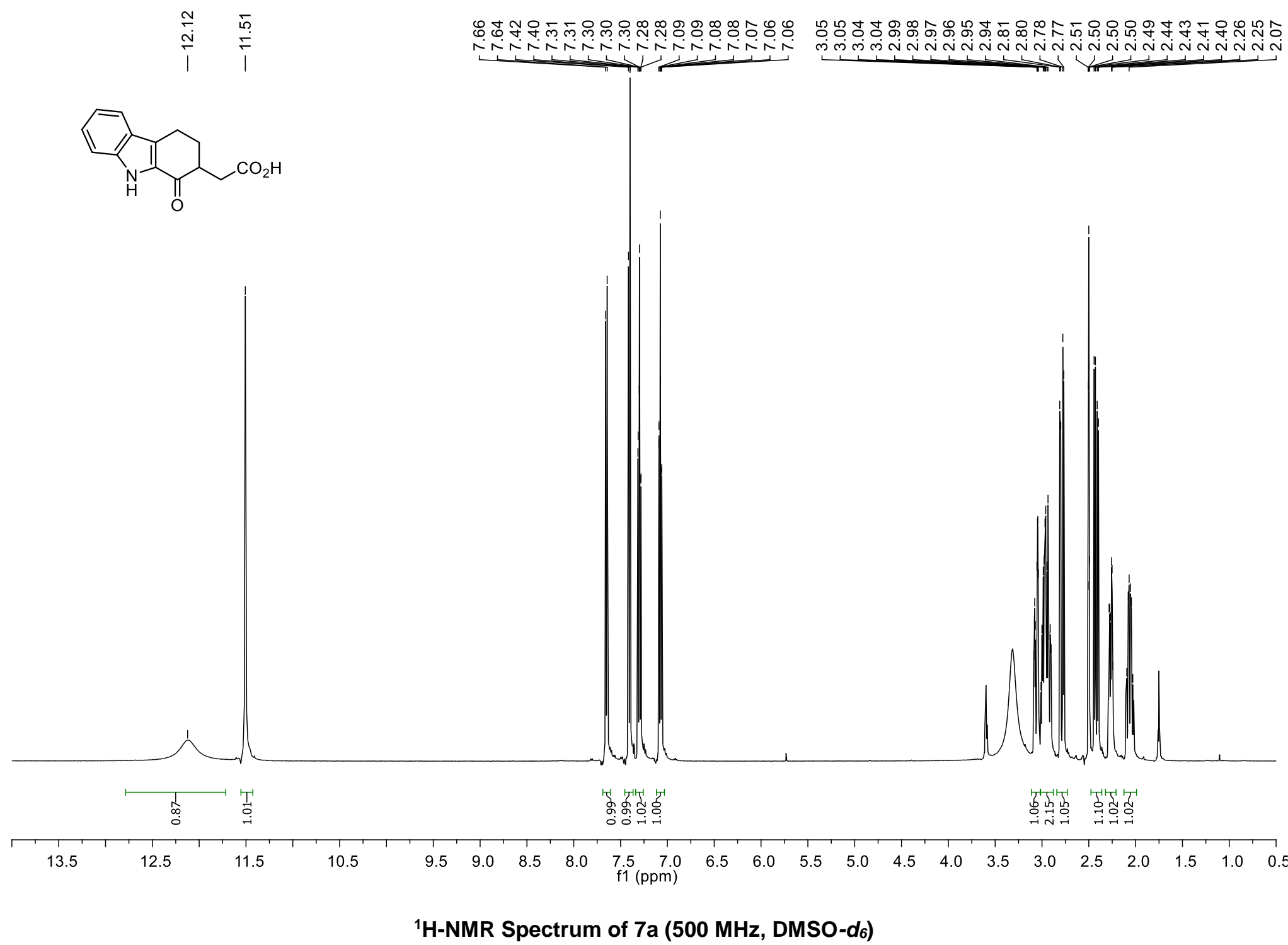




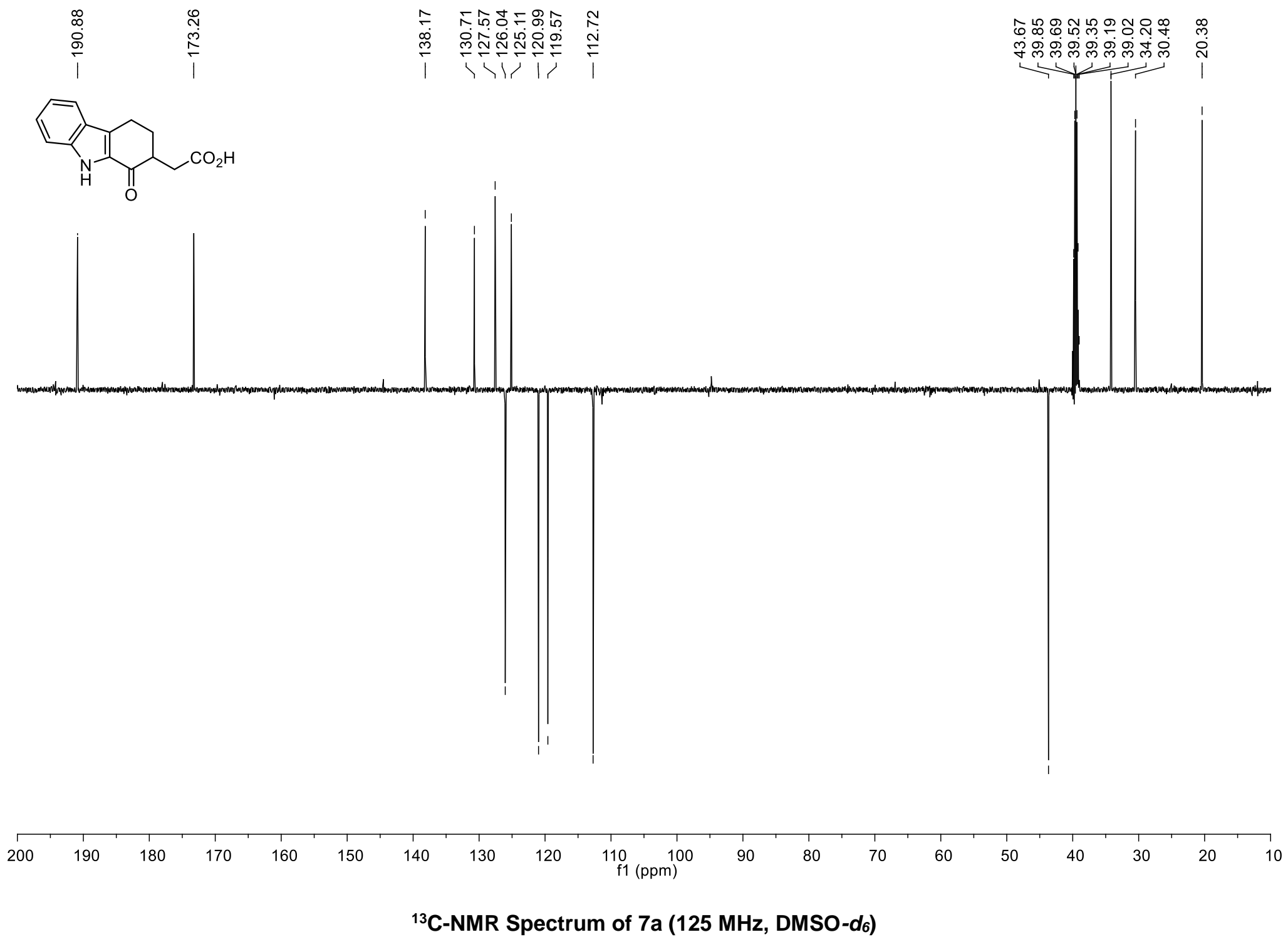



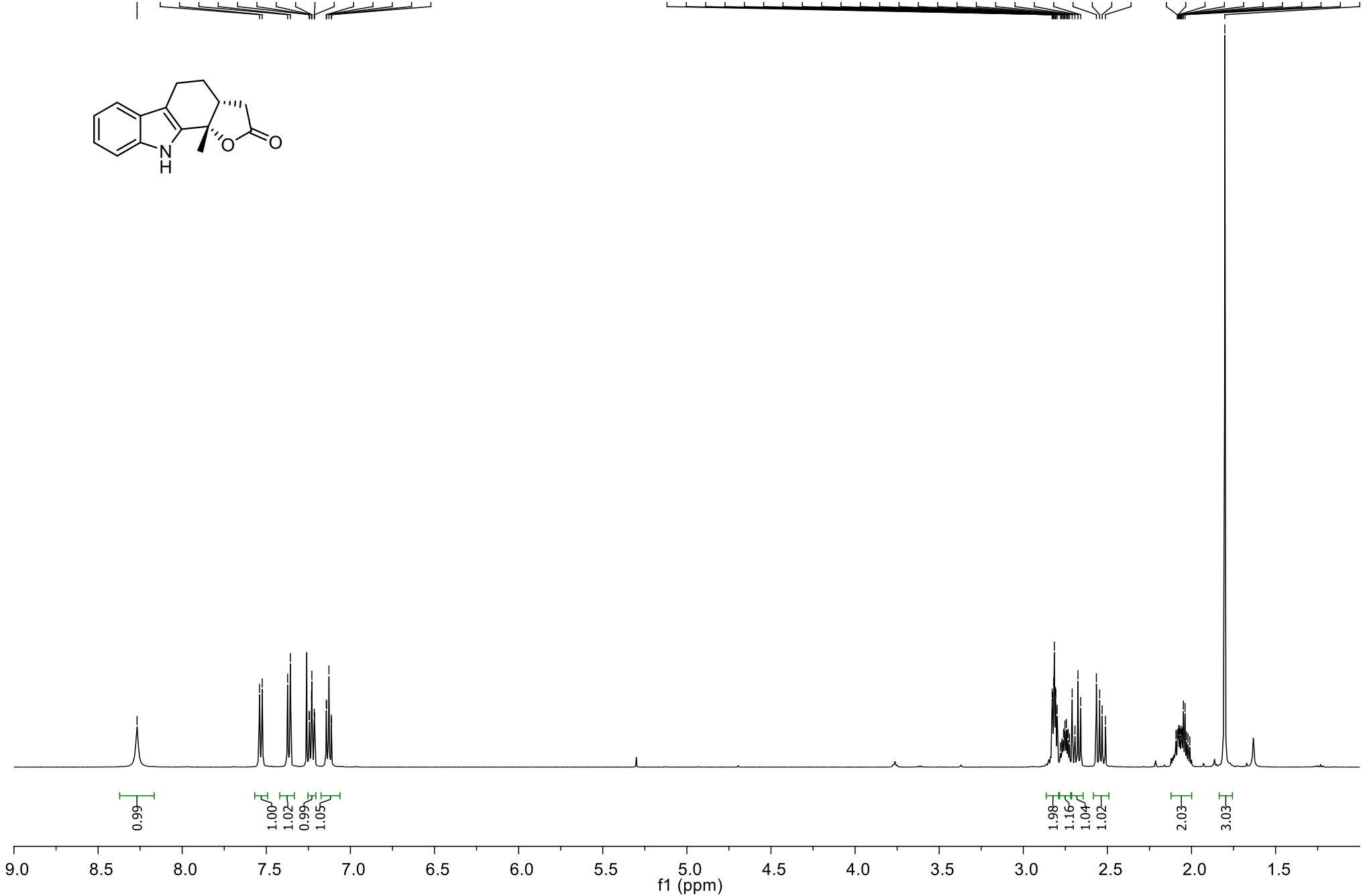

${ }^{1} \mathrm{H}-\mathrm{NMR}$ Spectrum of $9 \mathrm{a}\left(500 \mathrm{MHz}, \mathrm{CDCl}_{3}\right)$ 


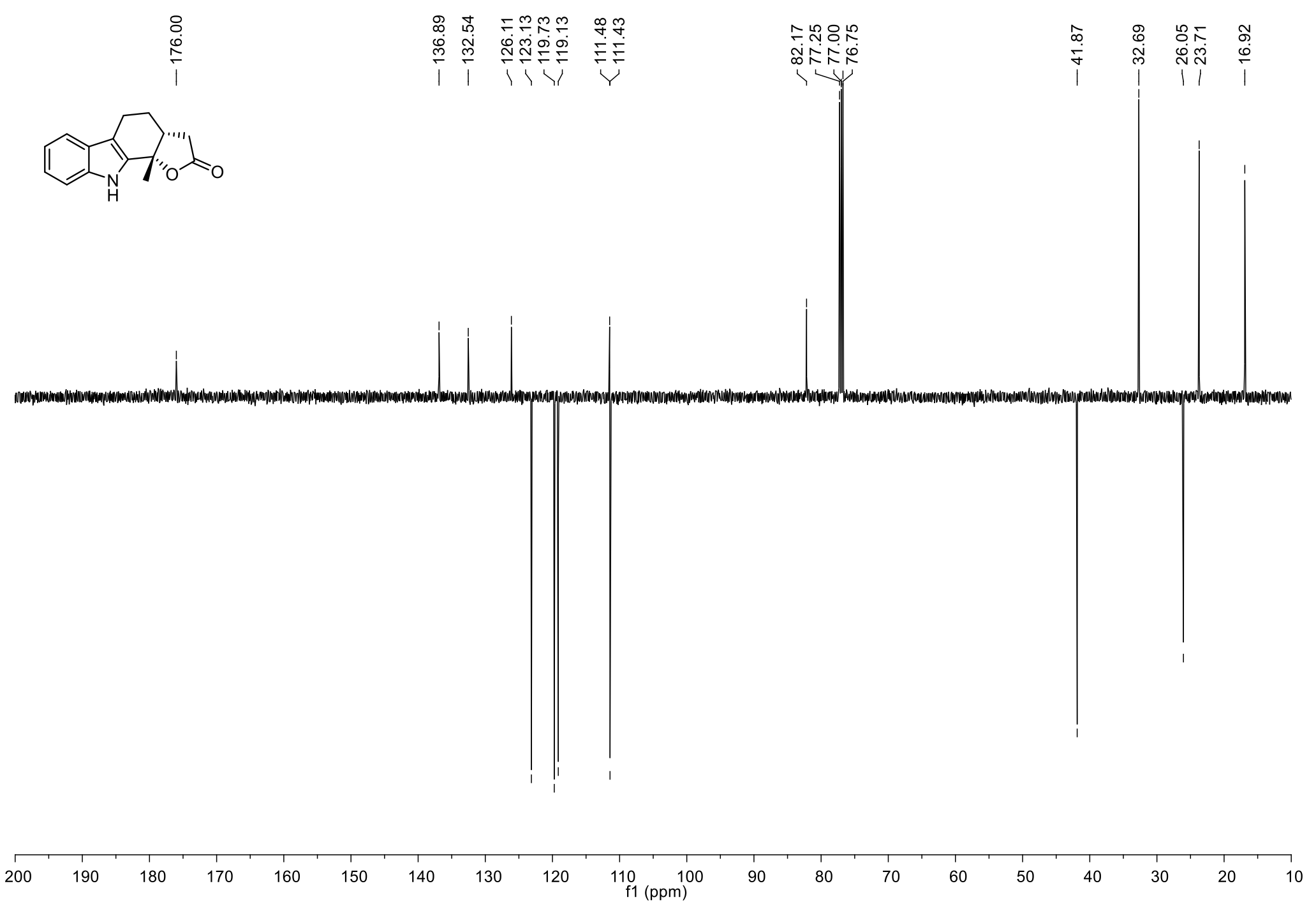

${ }^{13} \mathrm{C}$-NMR Spectrum of $9 \mathrm{a}\left(125 \mathrm{MHz}, \mathrm{CDCl}_{3}\right)$ 


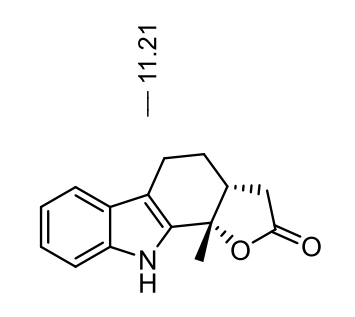

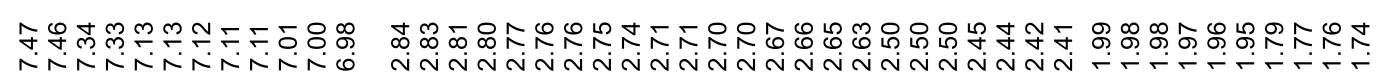
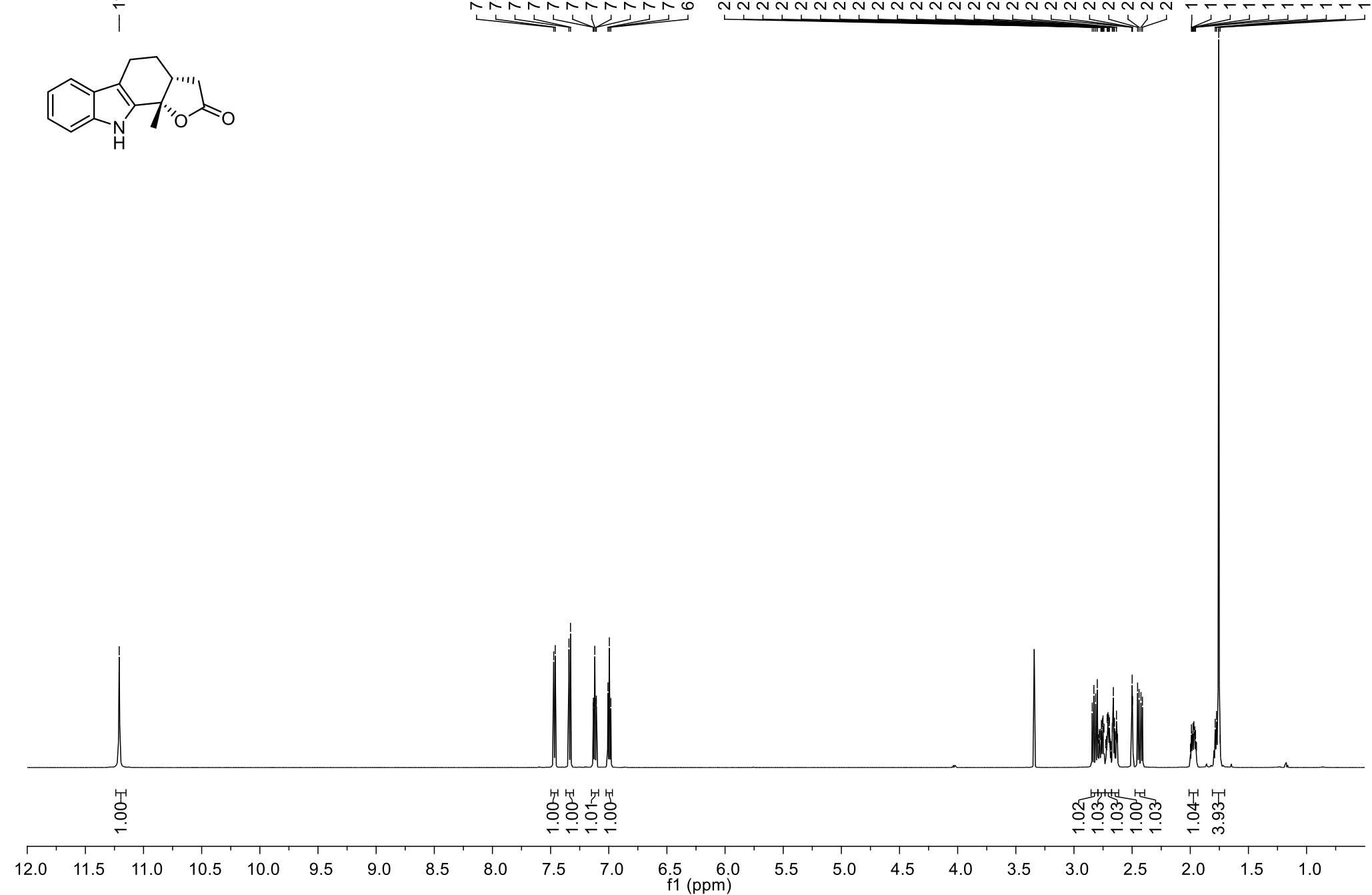

${ }^{1} \mathrm{H}-\mathrm{NMR}$ Spectrum of $9 \mathrm{a}\left(600 \mathrm{MHz}\right.$, DMSO- $\left.d_{6}\right)$ 


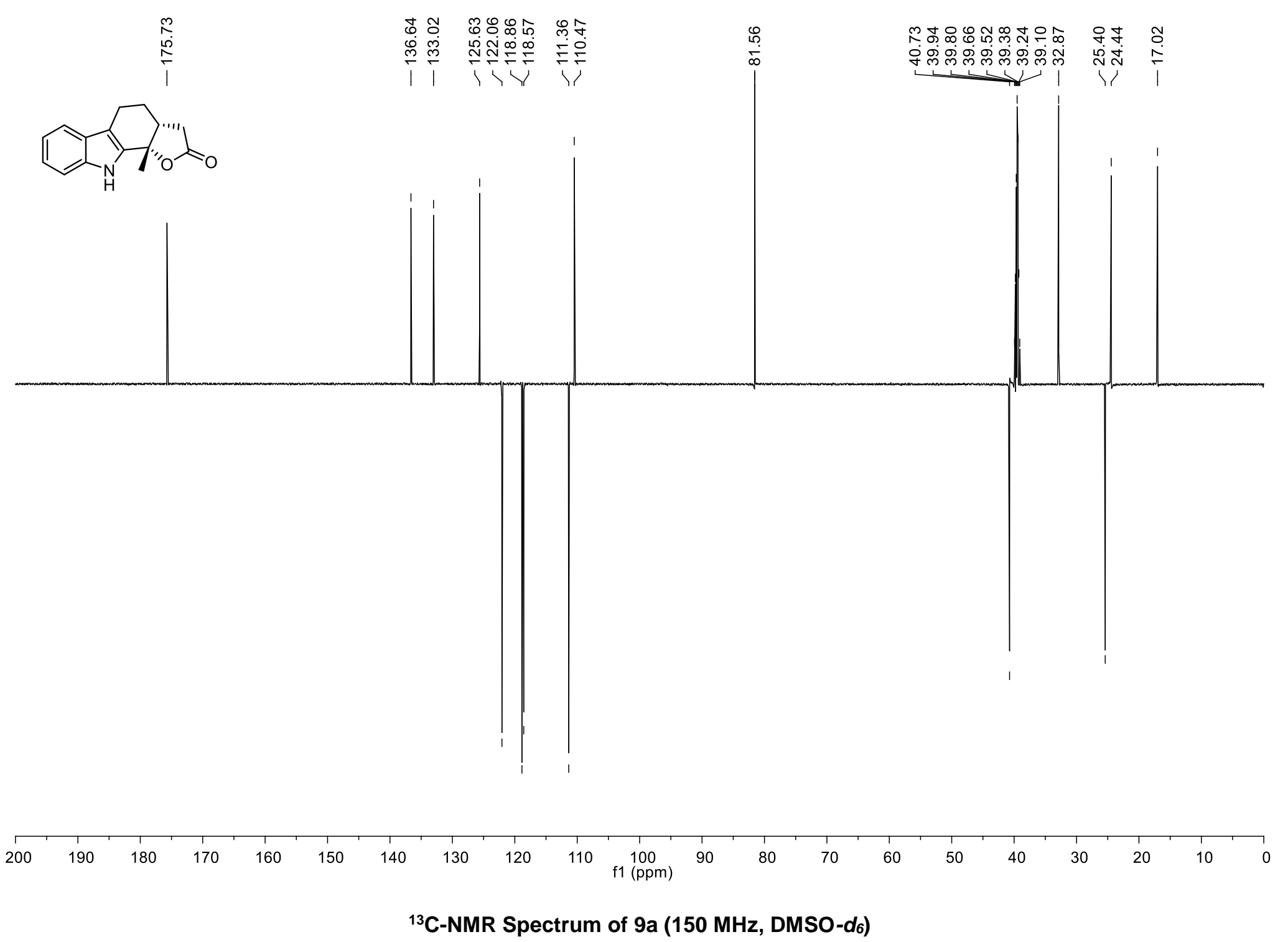




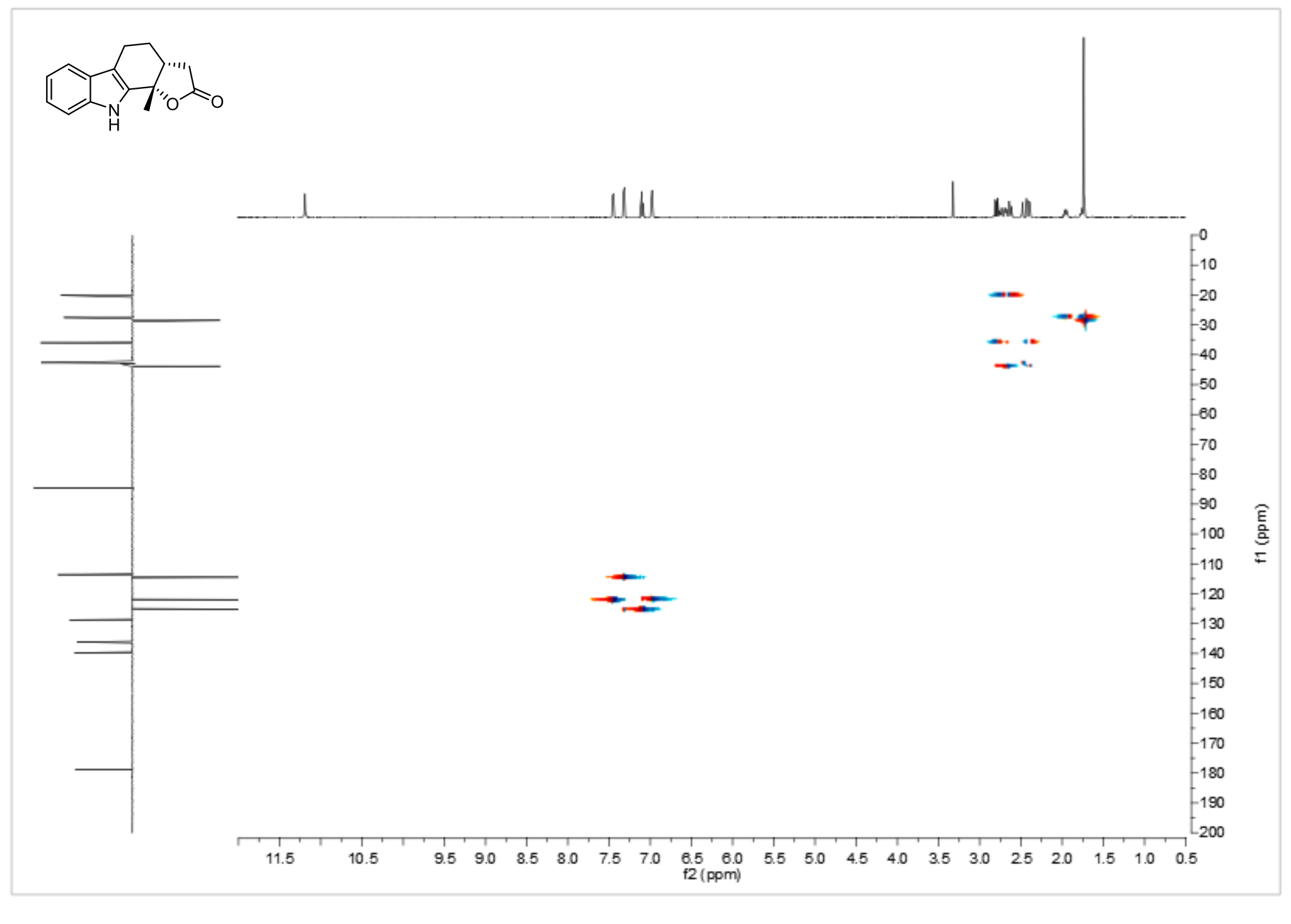

HSQC Spectrum of 9a (DMSO-d $d_{6}$ ) 


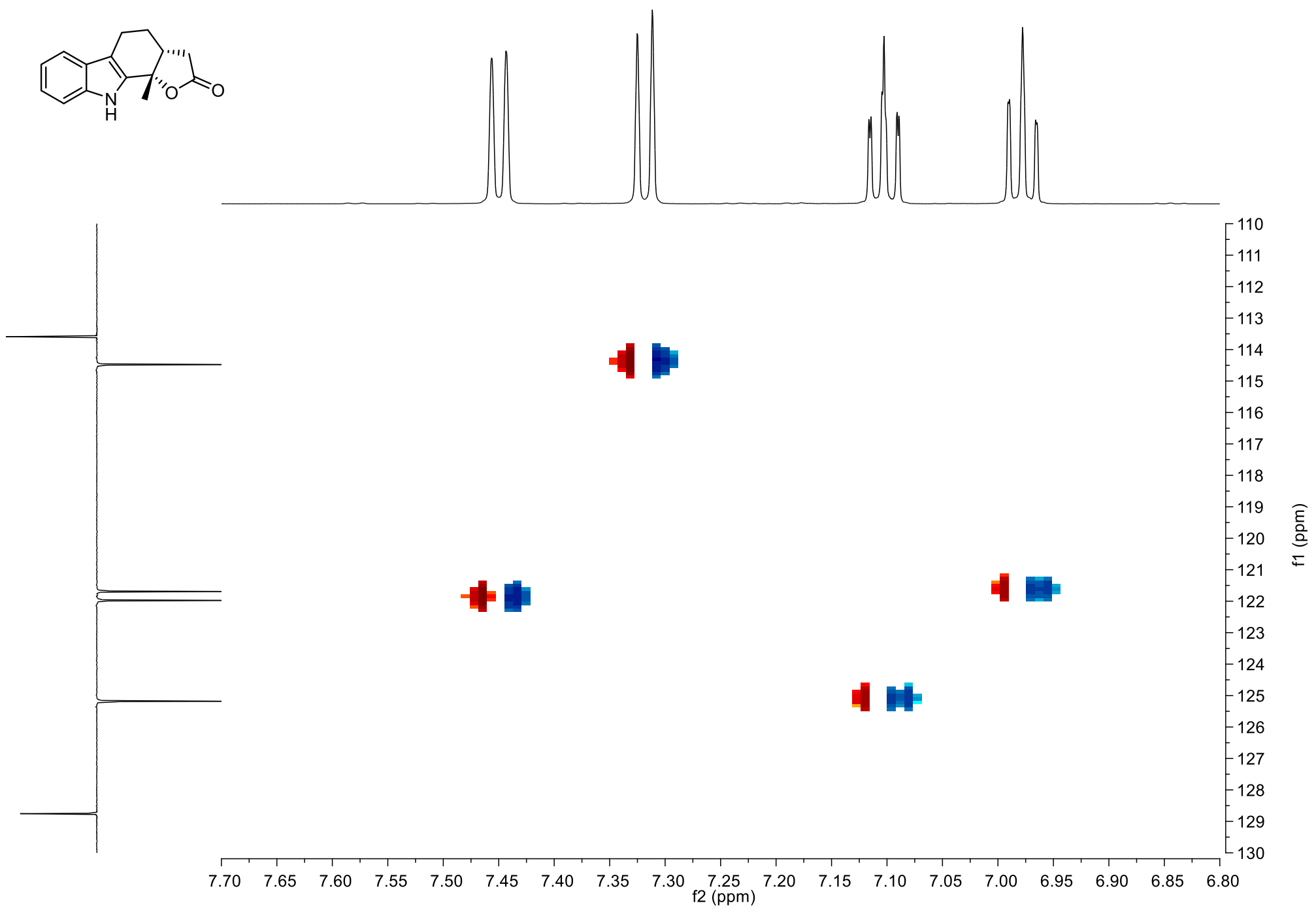

HSQC Spectrum of 9a (DMSO- $d_{6}$ ) 


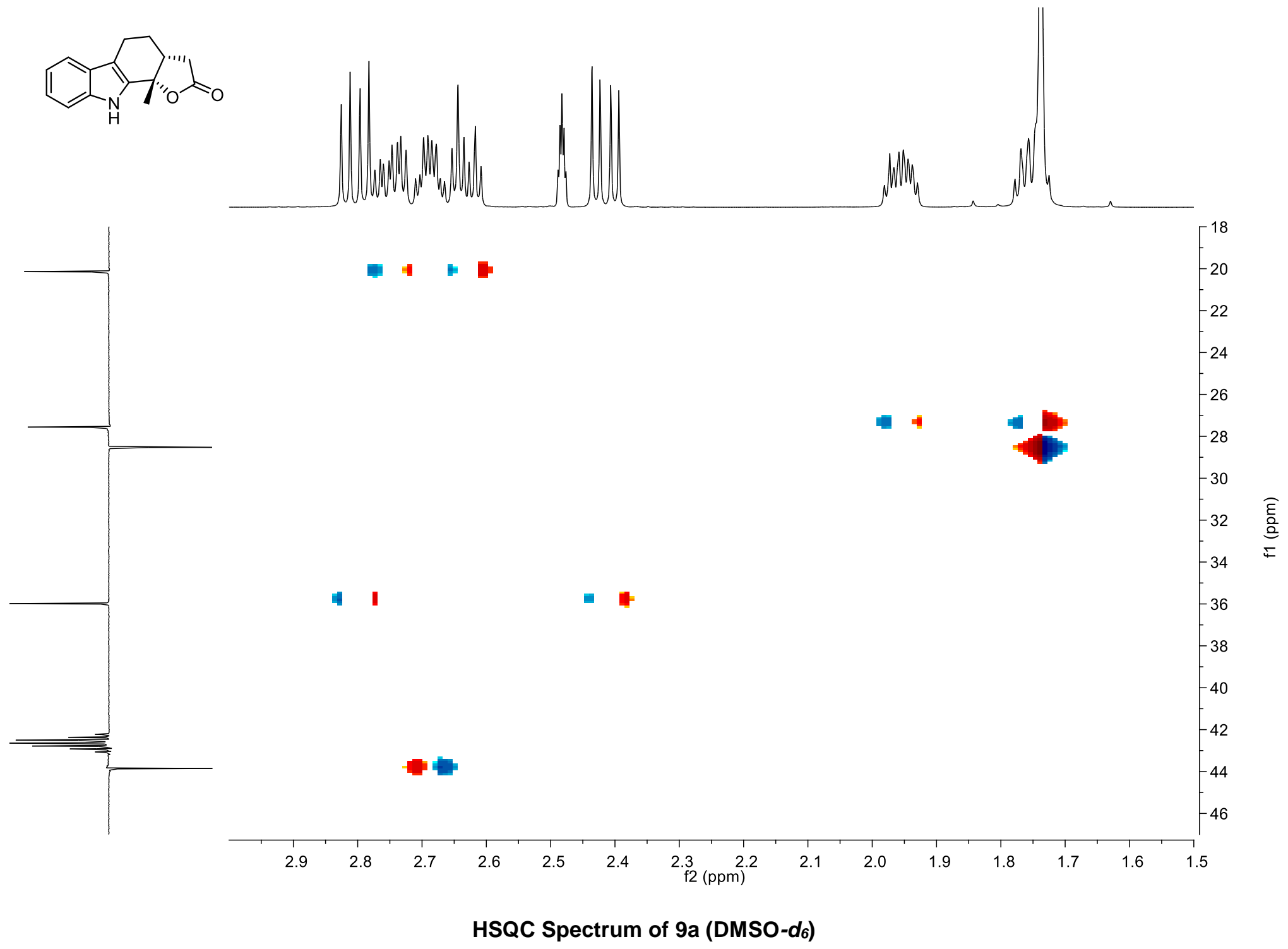




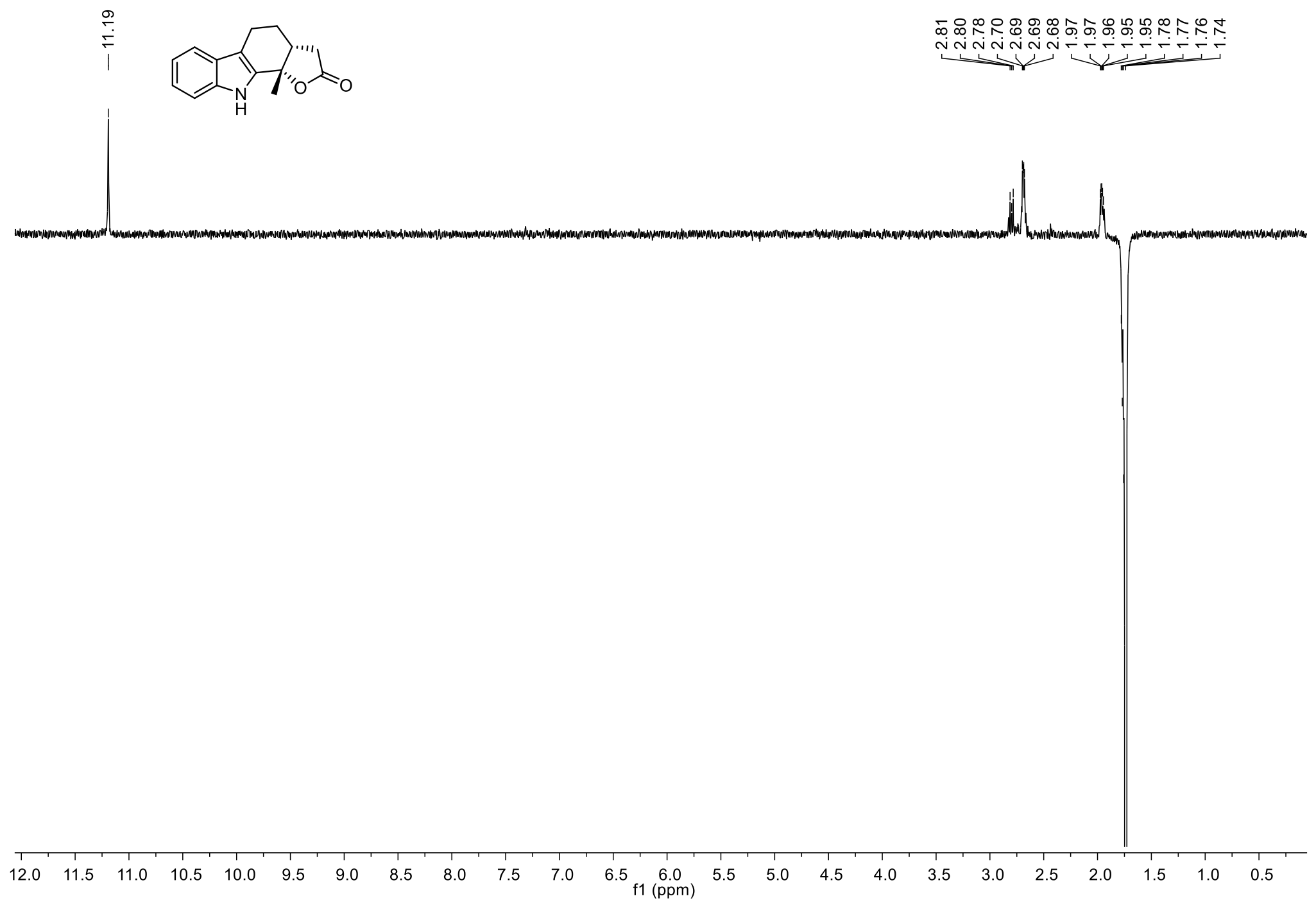

NOE-DIFF Spectrum of $9 \mathrm{a}\left(600 \mathrm{MHz}\right.$, DMSO- $\left.\boldsymbol{d}_{6}\right)$ : Enhancement of $\boldsymbol{H - 2}, \boldsymbol{H}_{\mathbf{a}}-3$ and $\boldsymbol{H 1 0}$ signals after irradiation of $\mathrm{C}_{3}$ signal at $1.72 \mathrm{ppm}$.

S19 
Table S1. NMR spectral data of compound 13.

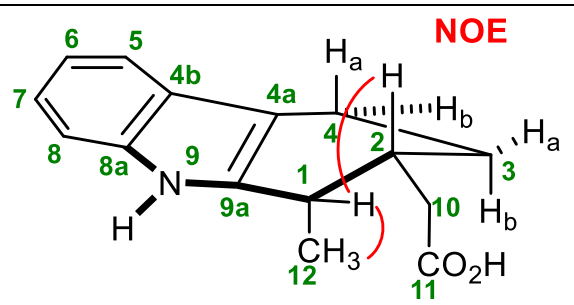

13

\begin{tabular}{|c|c|c|c|c|c|}
\hline $\mathrm{C \#}$ & $\delta_{\mathrm{C}}$ & $\delta_{\mathrm{H}}$ & Mult. & COSY & NOE-DIFF \\
\hline 1 & 30.7 & $3.04(\mathrm{qd}, J=12.4,6.3 \mathrm{~Hz}, 1 \mathrm{H}, H-1)$ & $\mathrm{CH}$ & $H-12, H-2$ & \\
\hline 2 & 35.7 & $2.34(\mathrm{~d}, J=3.7 \mathrm{~Hz}, 1 \mathrm{H}, H-2)$ & $\mathrm{CH}$ & $H-1,2 \times H-3,2 \times H-10$ & $H-12, H-2$ \\
\hline 3 & 24.8 & $1.75-1.68(\mathrm{~m}, 1 \mathrm{H}, H-3)$ & $\mathrm{CH}_{2}$ & $H-3,2 \times H-4, H-2$ & \\
\hline 3 & - & $1.59(\mathrm{dd}, J=10.9,4.3 \mathrm{~Hz}, 1 \mathrm{H}, H-3)$ & & $H-3,2 \times H-4, H-2$ & \\
\hline 4 & 20.6 & $2.69-2.60(\mathrm{~m}, 1 \mathrm{H}, H-4)$ & $\mathrm{CH}_{2}$ & $H-4,2 \times H-3$ & \\
\hline 4 & - & $2.57-2.51(\mathrm{~m}, 1 \mathrm{H}, H-4)$ & & $H-4,2 \times H-3$ & \\
\hline $4 \mathbf{a}$ & 106.9 & & $\mathrm{C}$ & & \\
\hline $4 b$ & 127.0 & & $\mathrm{C}$ & & \\
\hline 5 & 117.2 & $6.88(\mathrm{t}, J=7.4 \mathrm{~Hz}, 1 \mathrm{H})$ & $\mathrm{CH}$ & & \\
\hline 6 & 117.7 & $6.95(\mathrm{dd}, J=11.2,3.9 \mathrm{~Hz}, 1 \mathrm{H})$ & $\mathrm{CH}$ & & \\
\hline 7 & 110.6 & $7.28(\mathrm{~d}, J=7.7 \mathrm{~Hz}, 1 \mathrm{H})$ & $\mathrm{CH}$ & & \\
\hline 8 & 119.8 & $7.23(\mathrm{~d}, J=7.9 \mathrm{~Hz}, 1 \mathrm{H})$ & $\mathrm{CH}$ & & \\
\hline \multirow[t]{2}{*}{$8 \mathbf{a}$} & 140.6 & $C-8 \mathrm{a}$ or $C-9 \mathrm{a}$ & $\mathrm{C}$ & & \\
\hline & & $10.70(\mathrm{~s}, N-\mathrm{H})$ & - & & \\
\hline $9 \mathbf{a}$ & 135.9 & $C-8 \mathrm{a}$ or $C-9 \mathrm{a}$ & $\mathrm{C}$ & & \\
\hline 10 & 41.0 & $2.11(\mathrm{dd}, J=14.3,7.1 \mathrm{~Hz}, 1 \mathrm{H}, H-10)$ & $\mathrm{CH}_{2}$ & & \\
\hline 10 & - & $2.01(\mathrm{dd}, J=14.3,7.8 \mathrm{~Hz}, 1 \mathrm{H}, H-10)$ & & & \\
\hline 11 & 176.7 & $C=\mathrm{O}$ & $\mathrm{C}$ & & \\
\hline 12 & 15.6 & $1.11(\mathrm{~d}, J=7.0 \mathrm{~Hz}, 3 \mathrm{H}, H-12)$ & $\mathrm{CH}_{3}$ & $H-1$ & \\
\hline
\end{tabular}




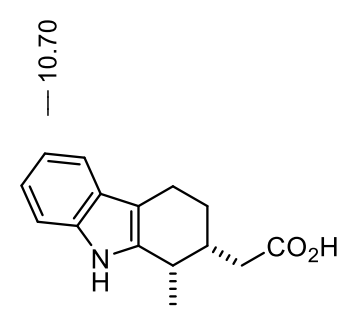

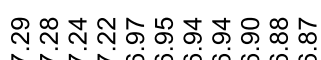

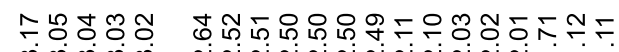
筮

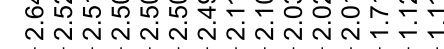

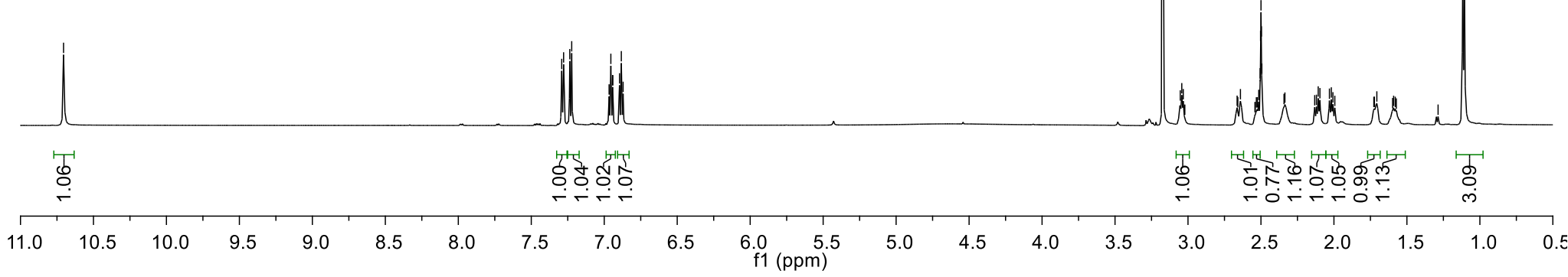

${ }^{1} \mathrm{H}-\mathrm{NMR}$ Spectrum of $13\left(600 \mathrm{MHz}, \mathrm{DMSO}-d_{6}\right)$ 


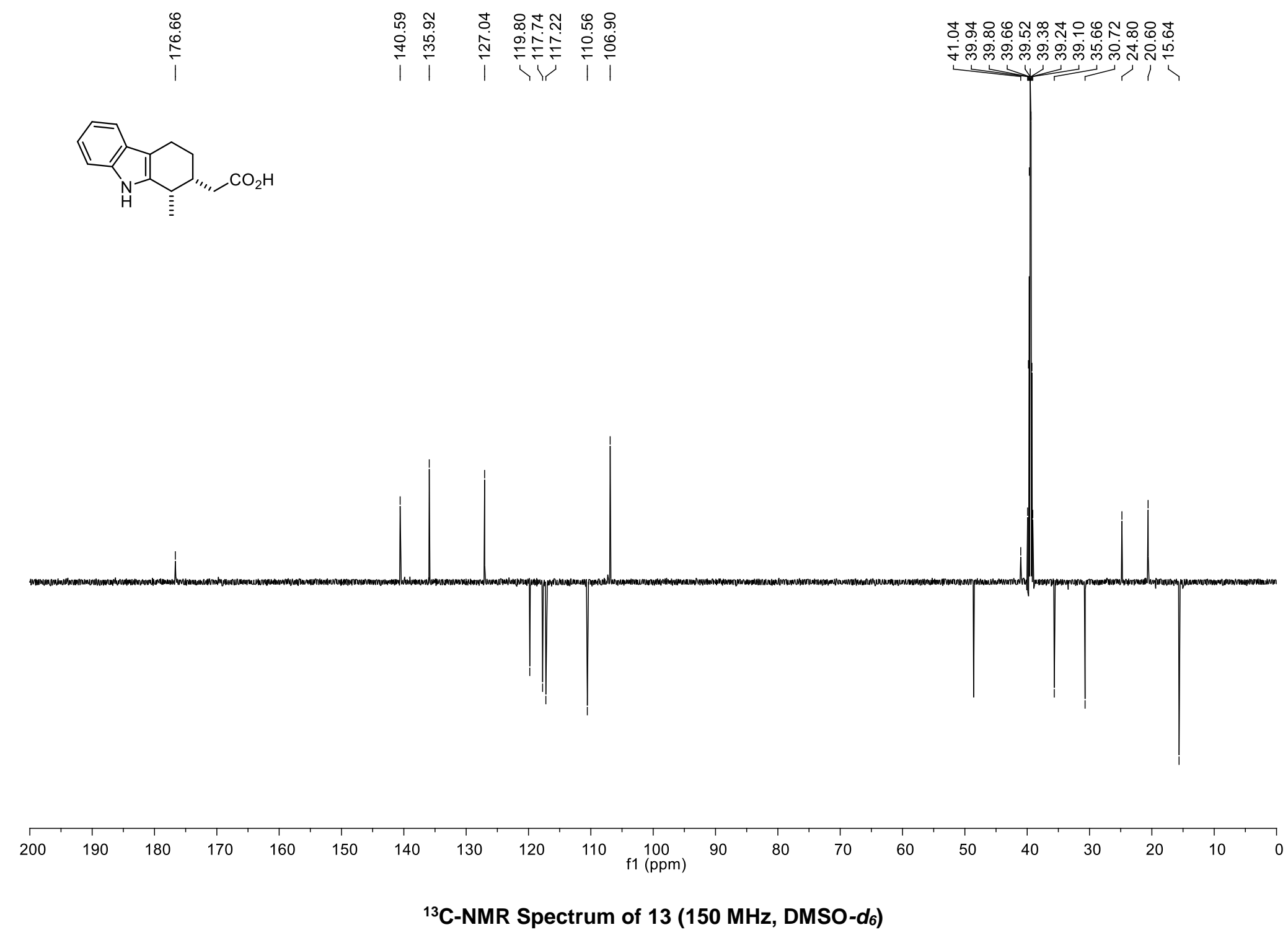




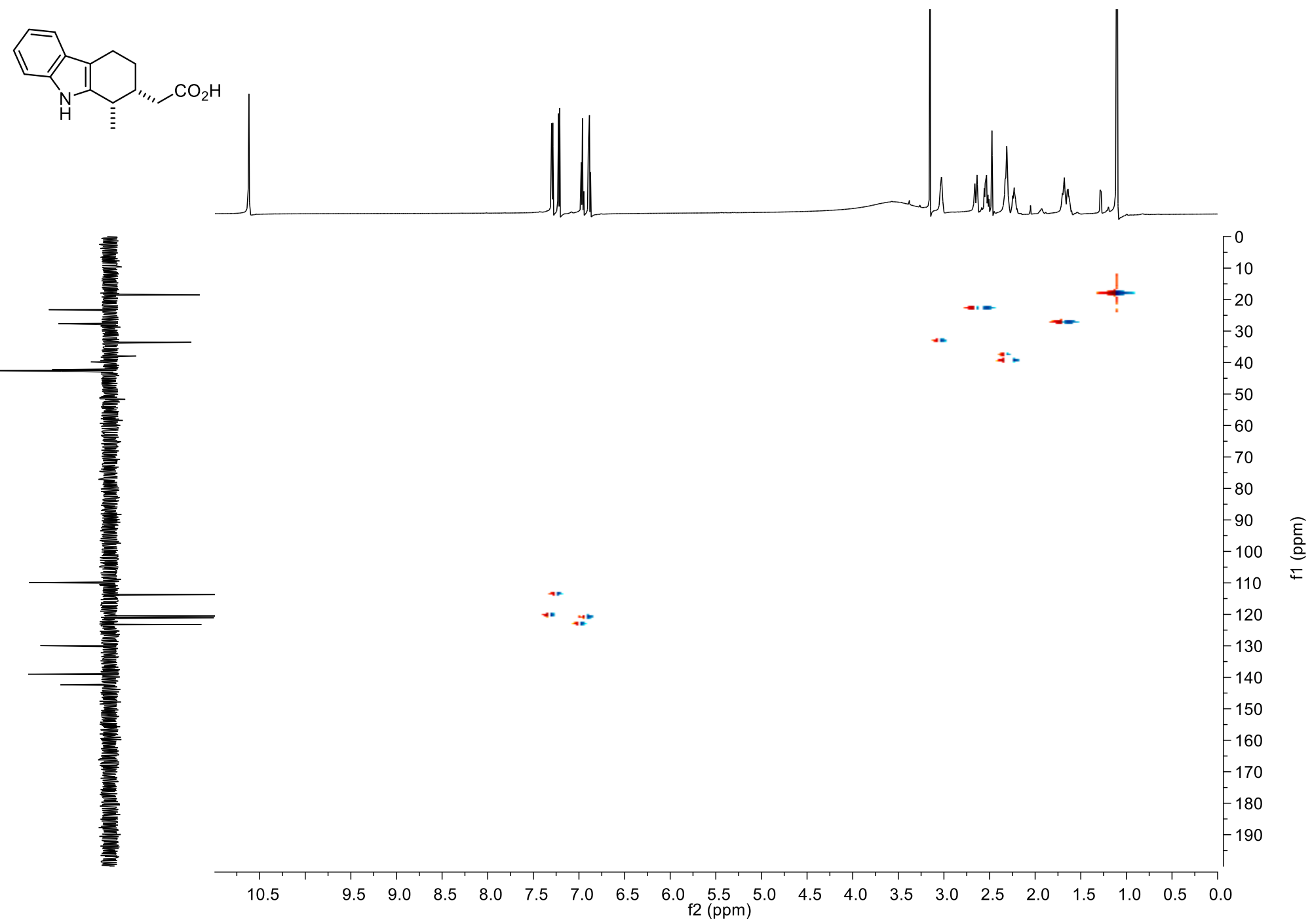

HSQC Spectrum of 13 


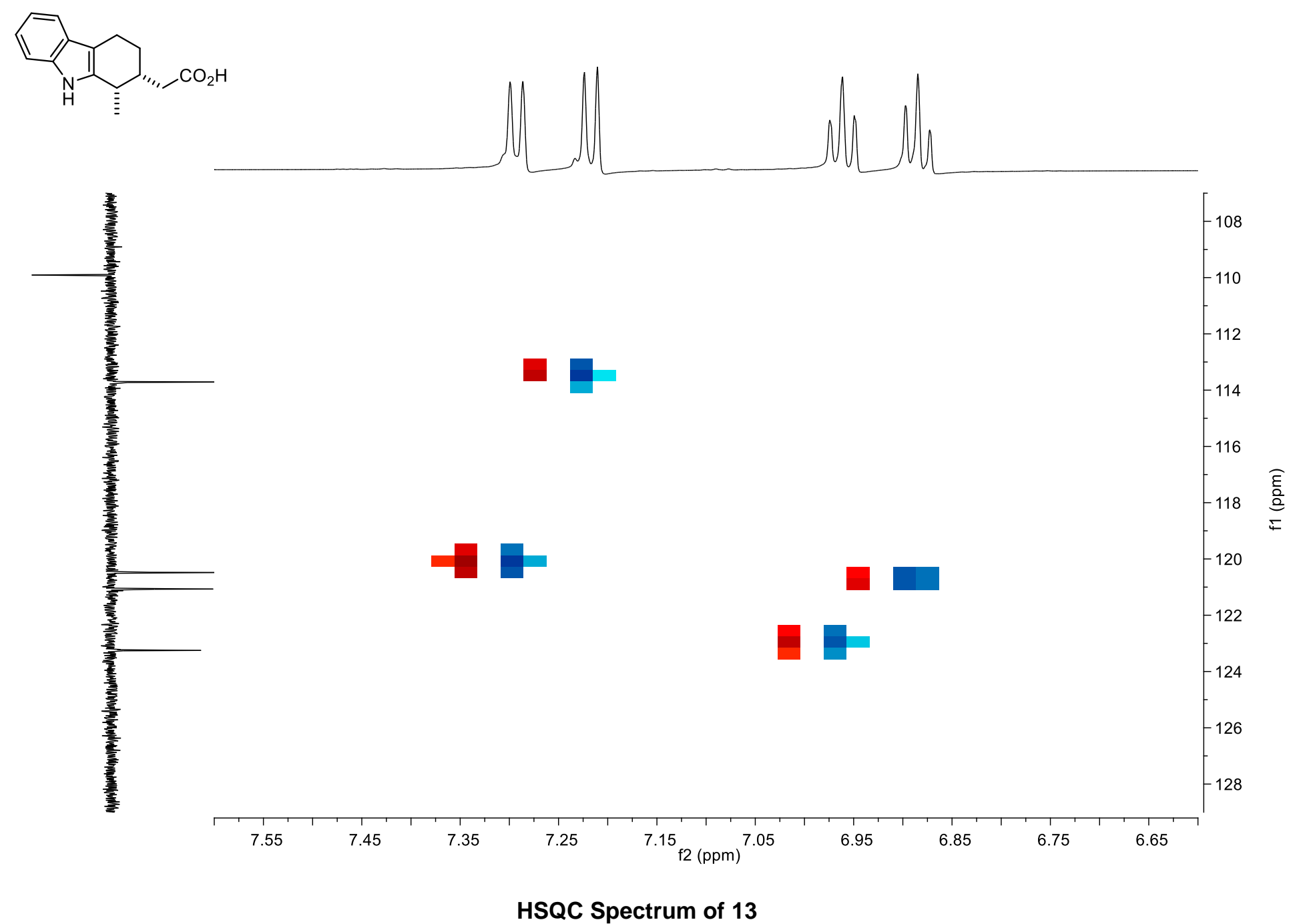




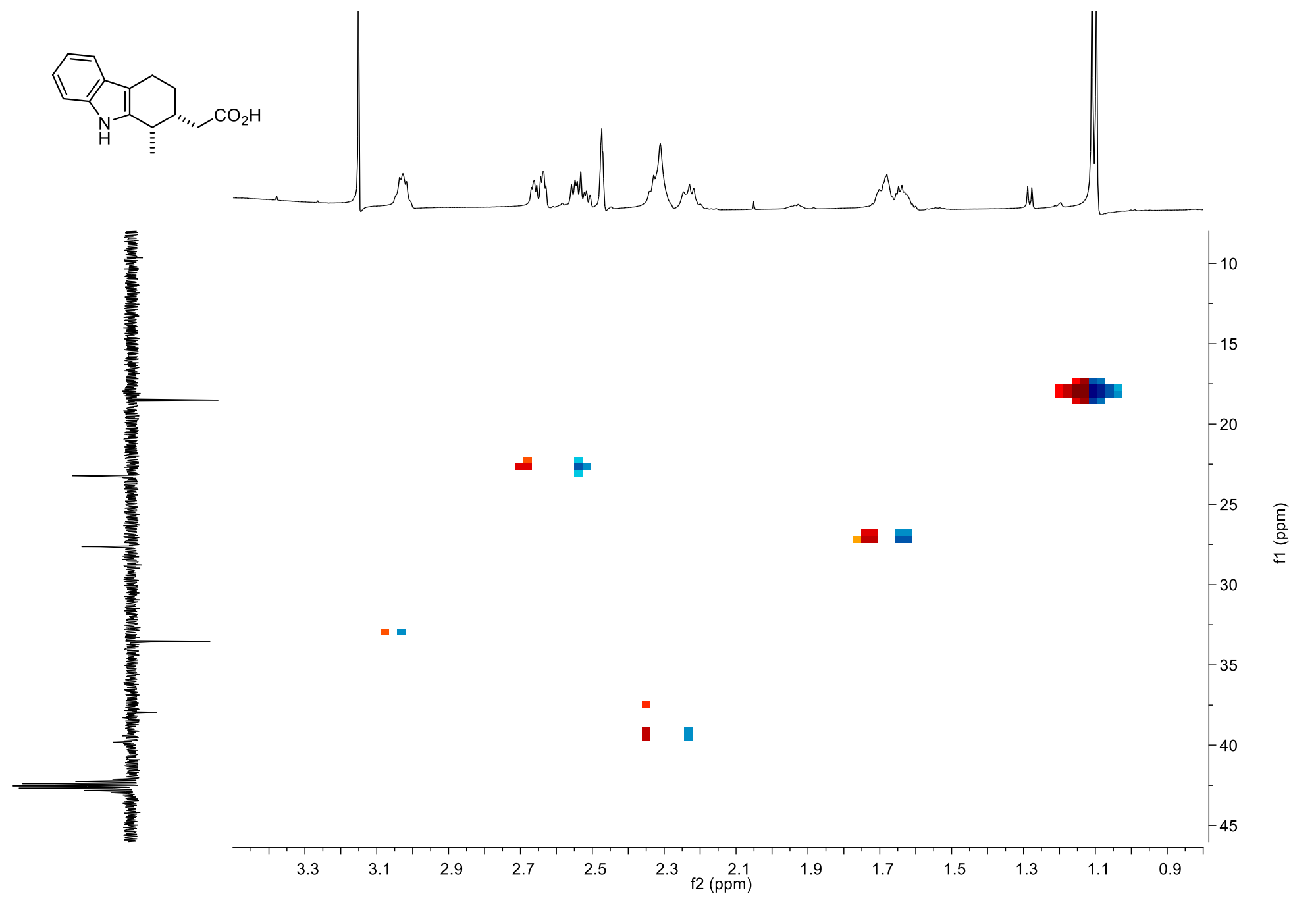

HSQC-DEPT Spectrum of 13 


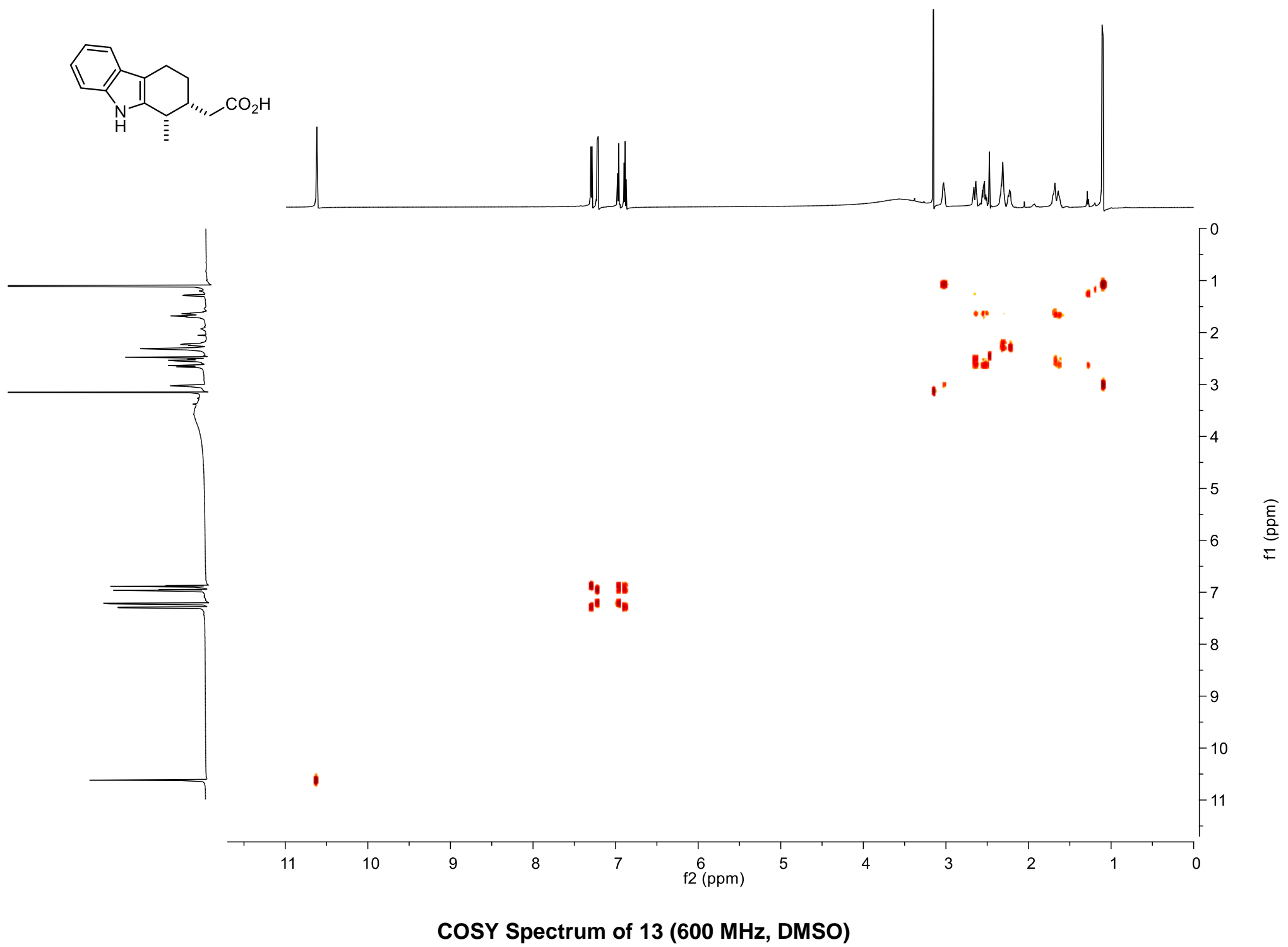




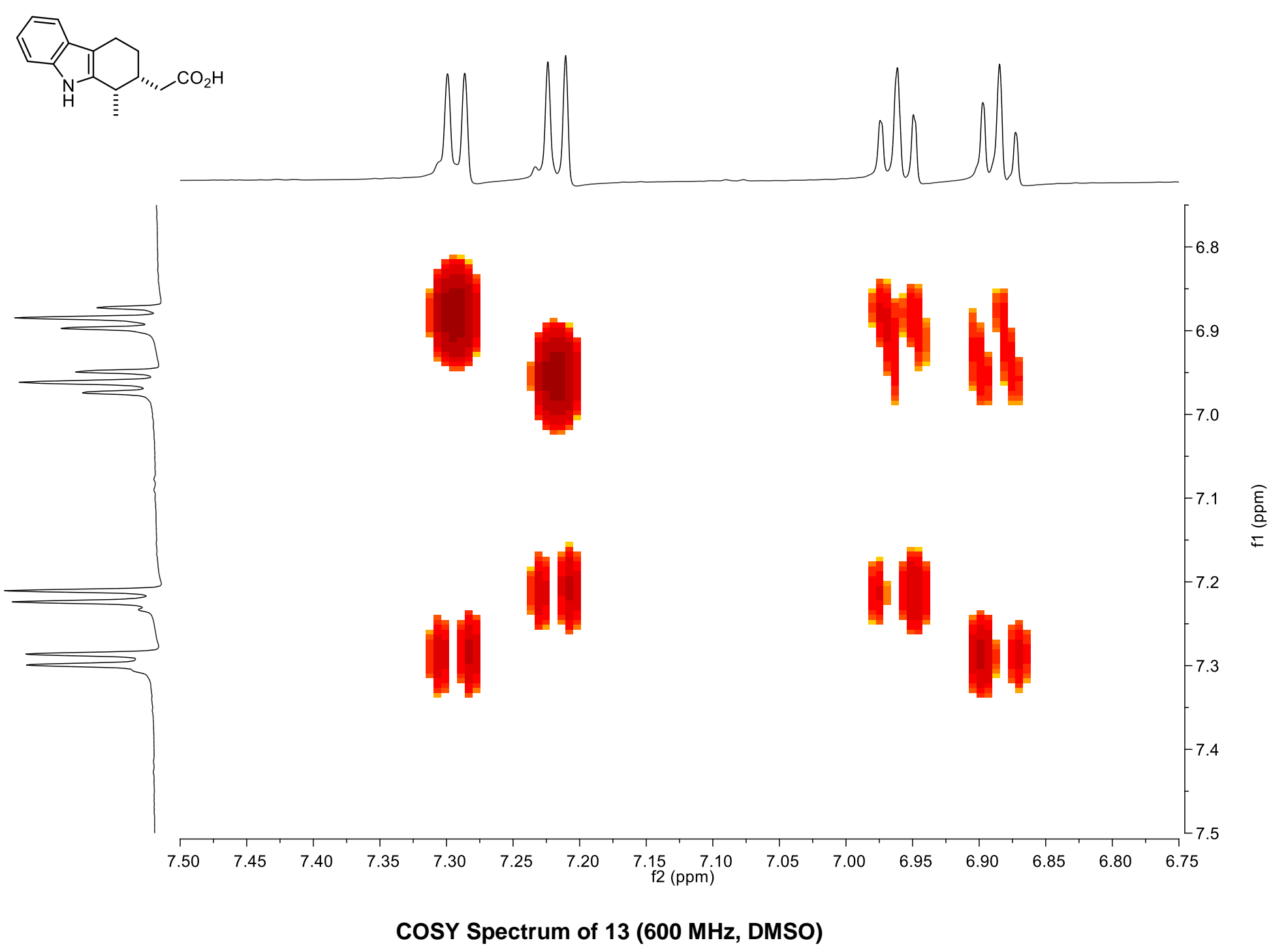




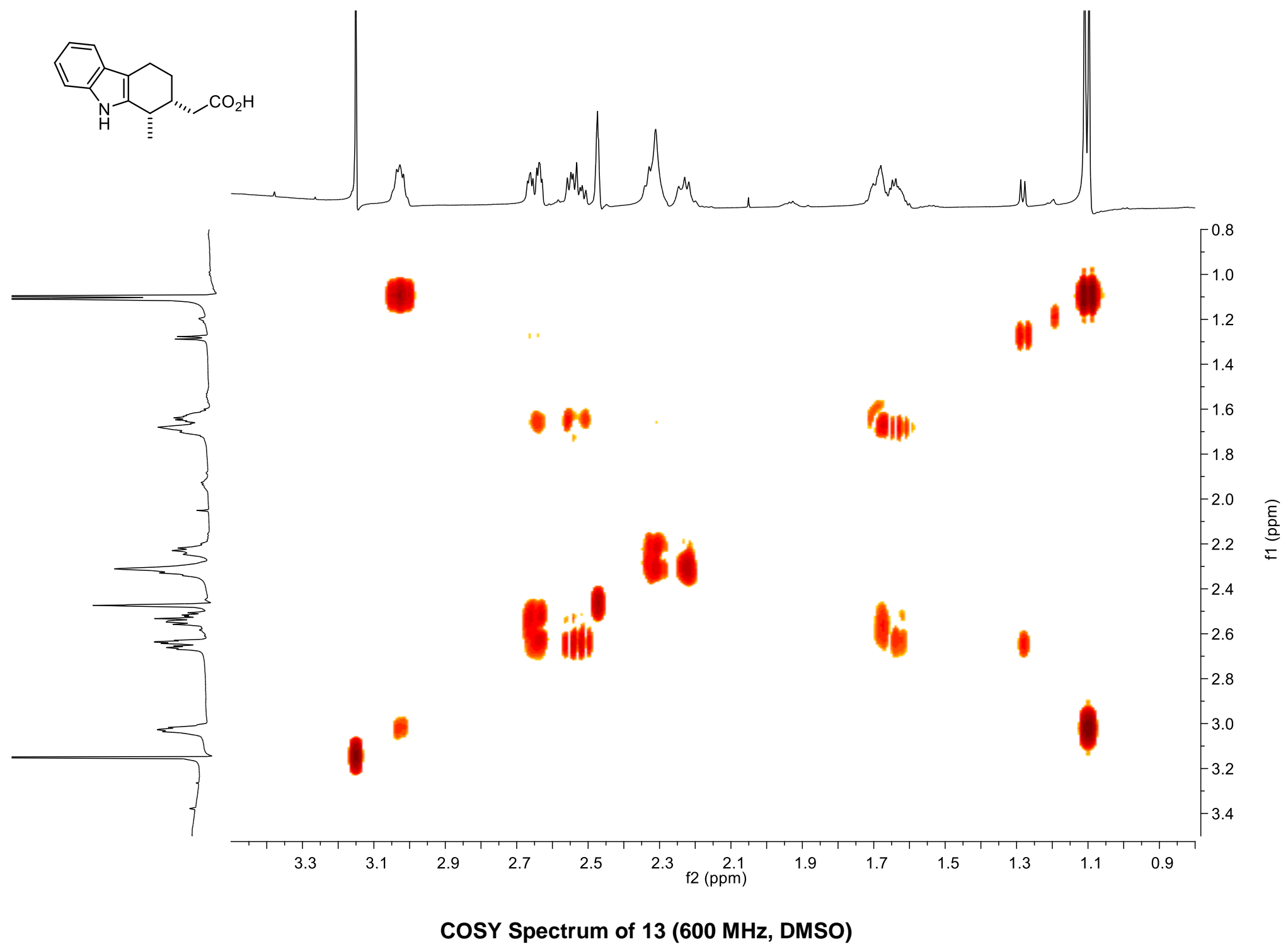




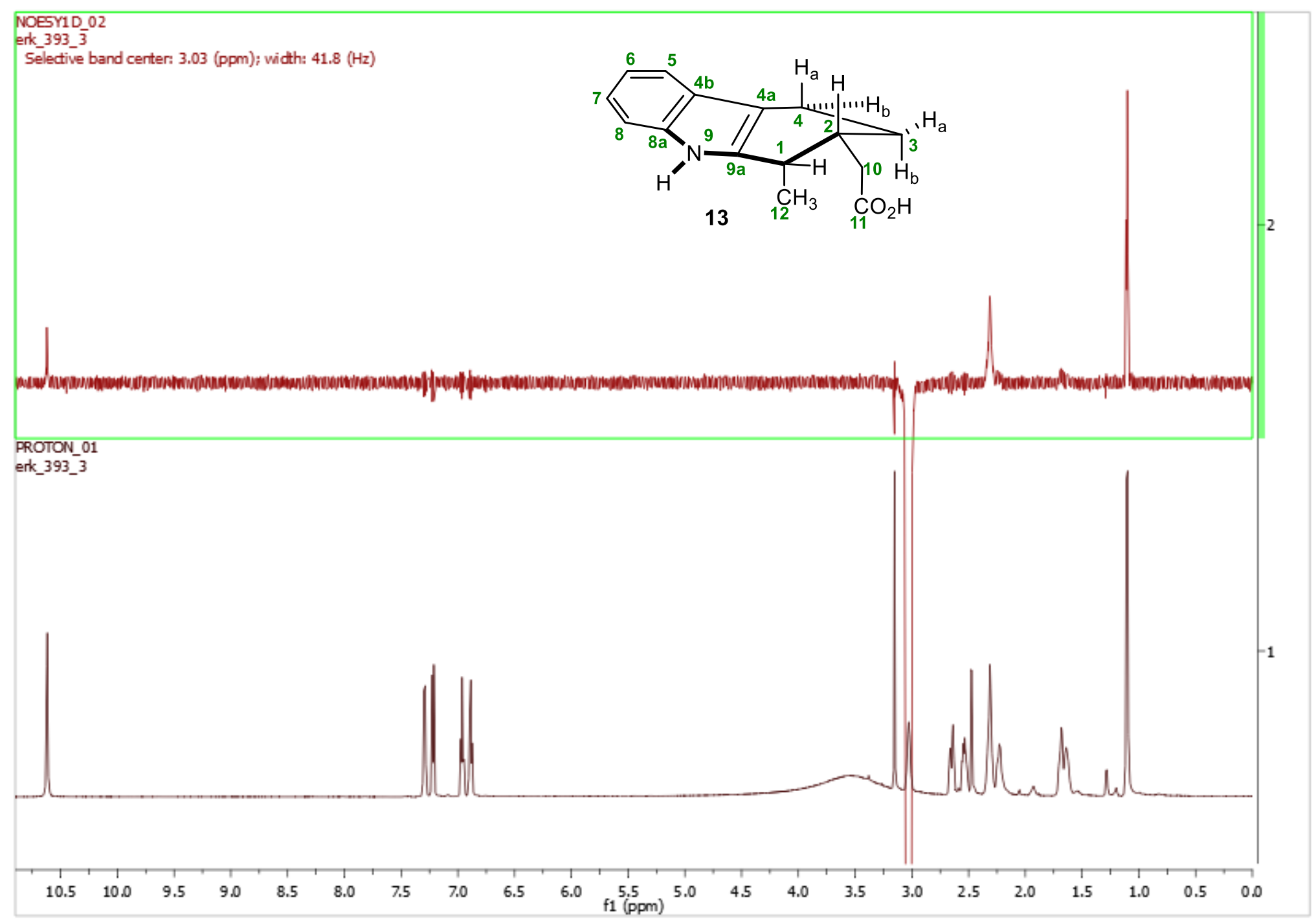

NOE-DIFF Spectrum of 13 (600 MHz, DMSO): Enhancement of $\boldsymbol{H - 1 2}$ and $\boldsymbol{H - 2}$ signals after irradiation of $\boldsymbol{H - 1}$ signal at 3.03 ppm. 


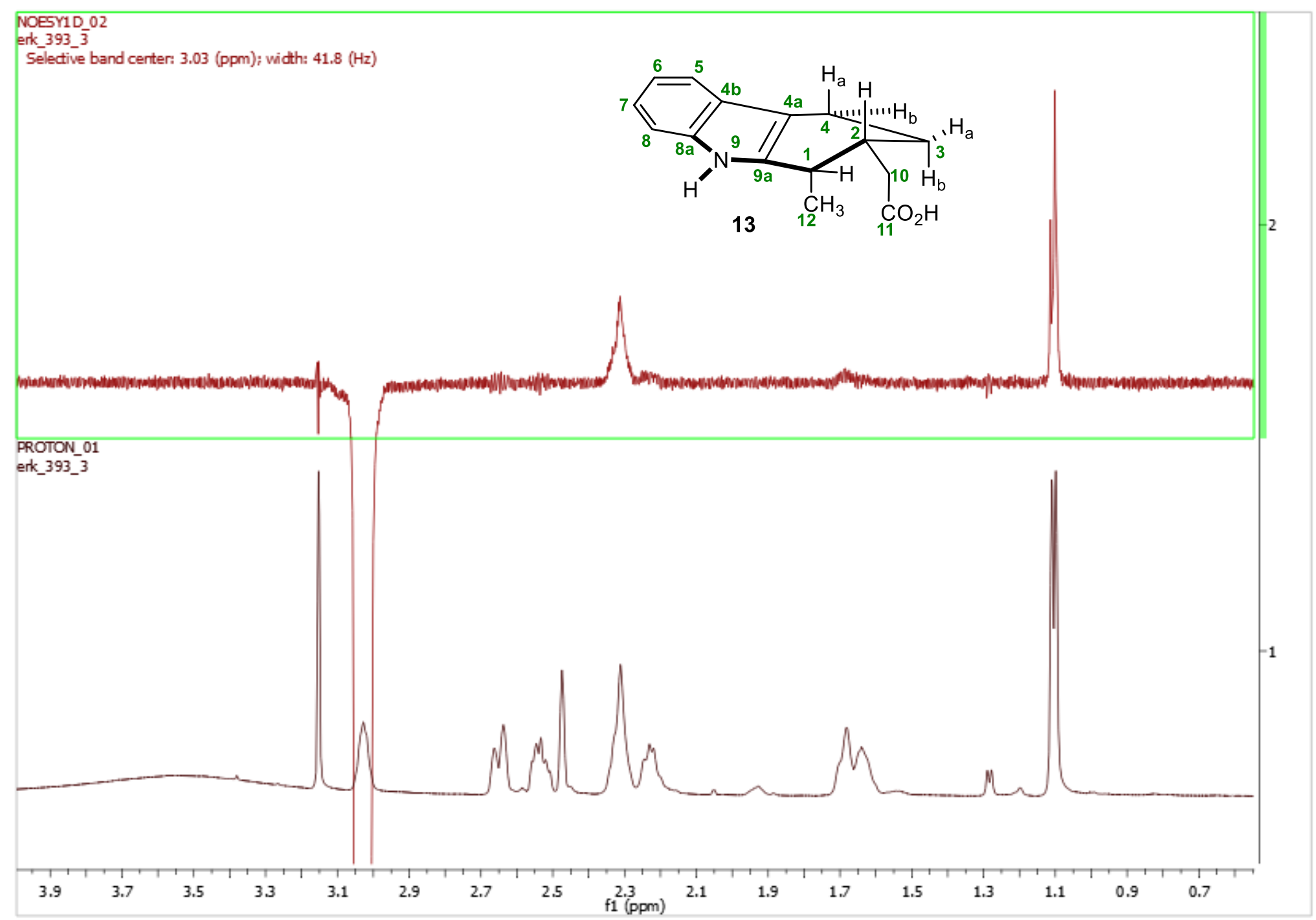

NOE-DIFF Spectrum of 13 (600 MHz, DMSO): Enhancement of $\boldsymbol{H - 1 2}$ and $\boldsymbol{H}-\mathbf{2}$ signals after irradiation of $\boldsymbol{H}-\mathbf{1}$ signal at 3.03 ppm 


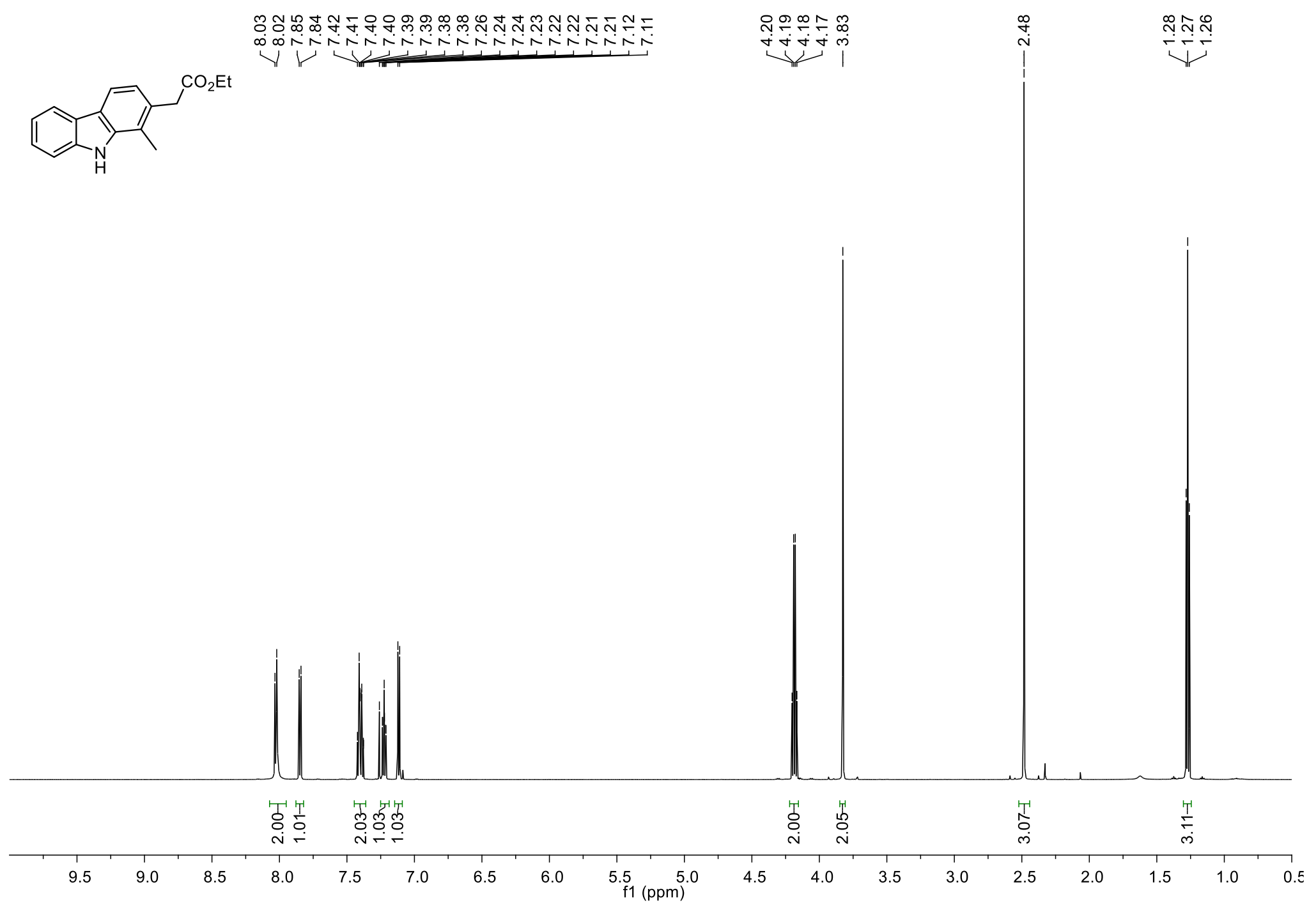

${ }^{1} \mathrm{H}-\mathrm{NMR}$ Spectrum of $15 \mathrm{a}\left(600 \mathrm{MHz}, \mathrm{CDCl}_{3}\right)$ 


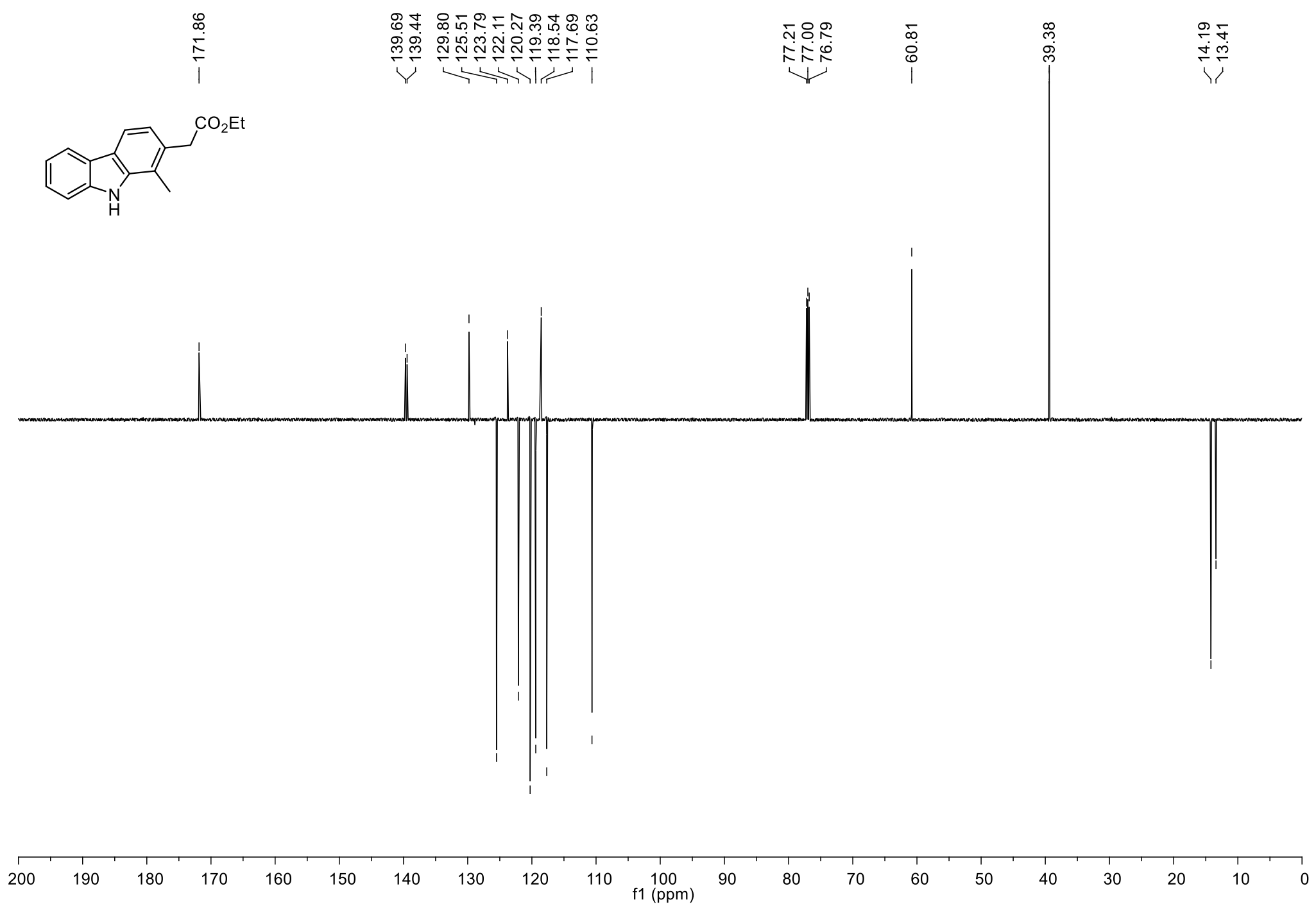

${ }^{13} \mathrm{C}-\mathrm{NMR}$ Spectrum of $15 \mathrm{a}\left(150 \mathrm{MHz}, \mathrm{CDCl}_{3}\right.$ ) 


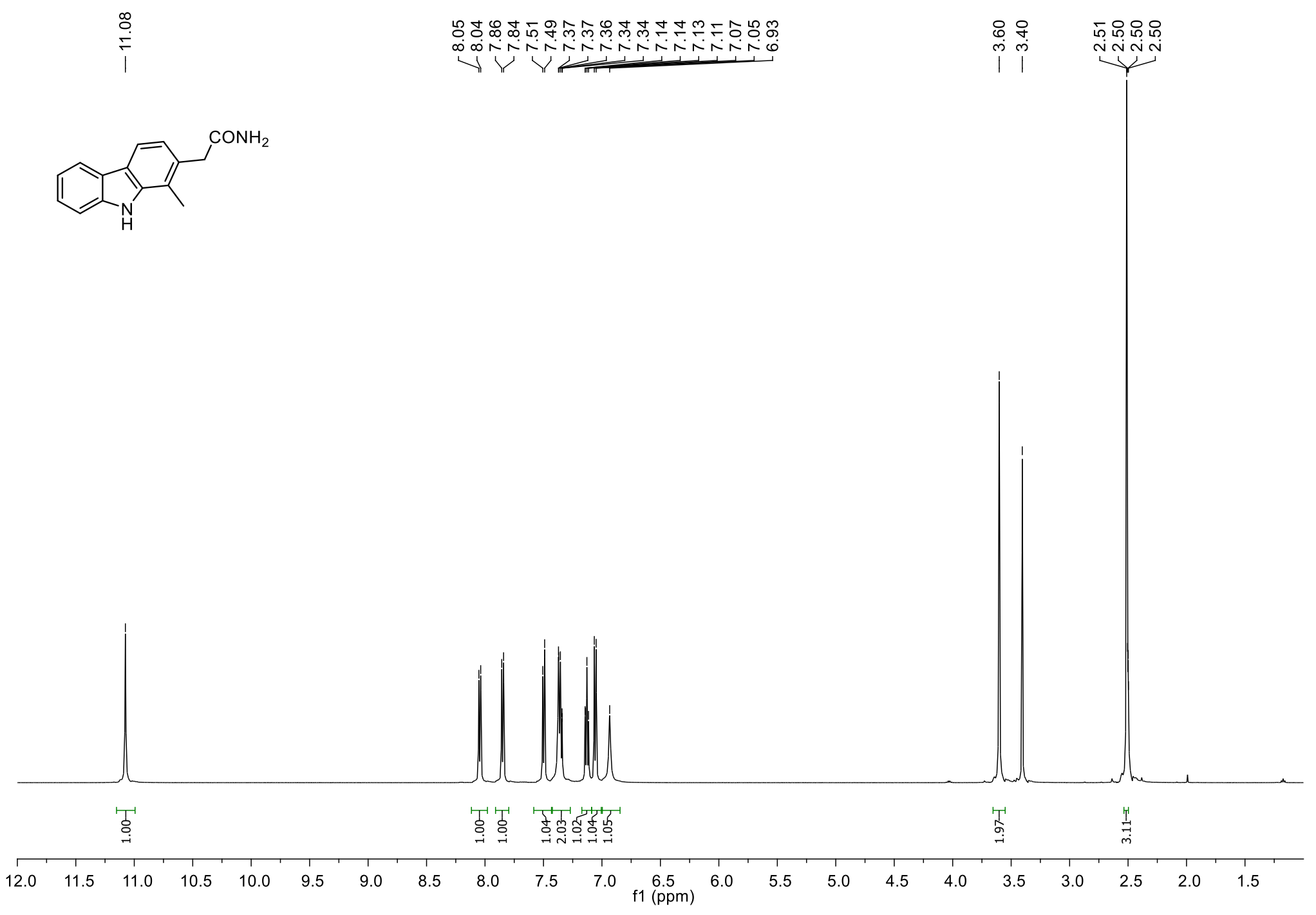

${ }^{1} \mathrm{H}-\mathrm{NMR}$ Spectrum of 17 (500 MHz, DMSO-d $)$ 


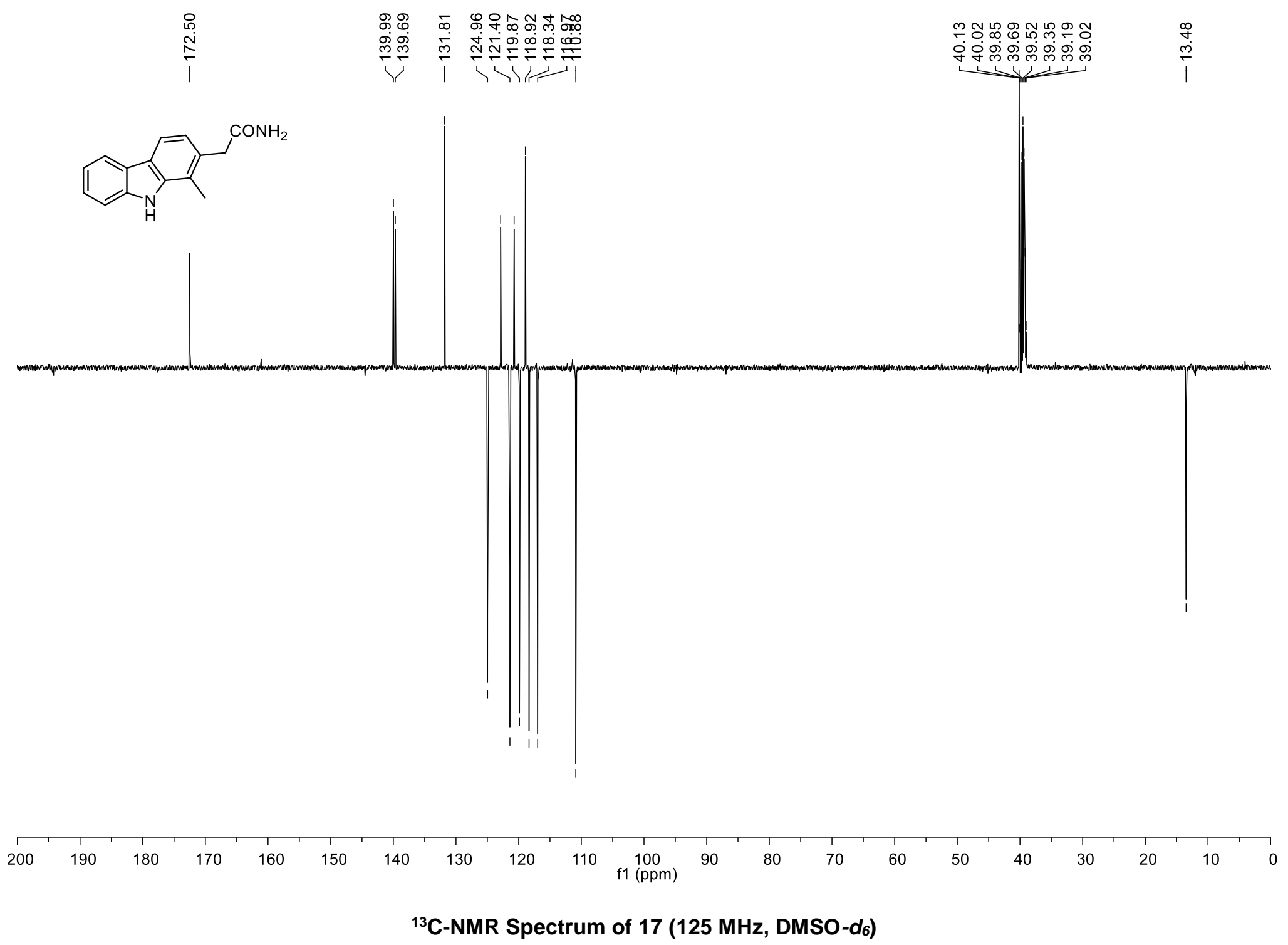



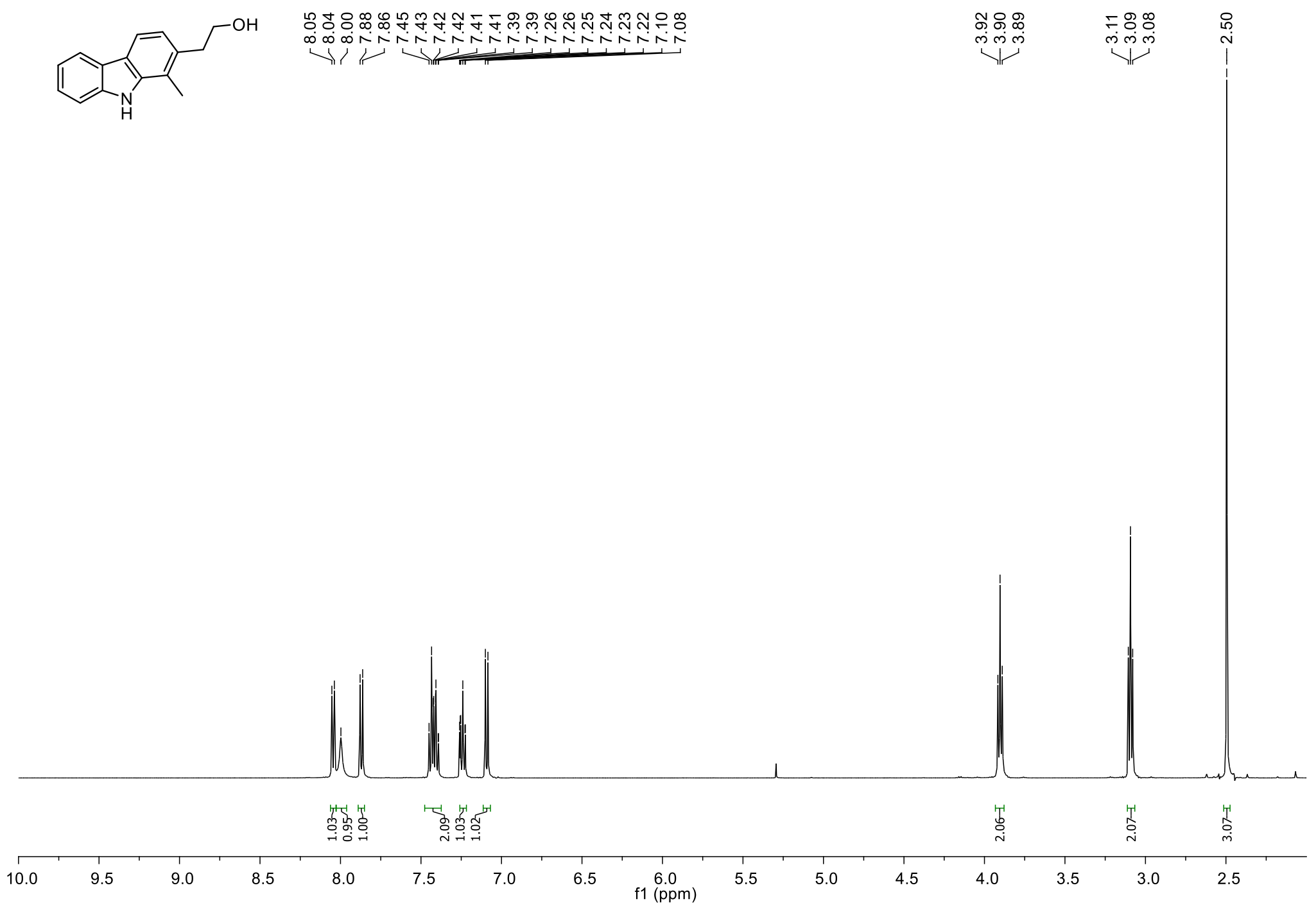

${ }^{1} \mathrm{H}-\mathrm{NMR}$ Spectrum of 21a (500 $\left.\mathrm{MHz}, \mathrm{CDCl}_{3}\right)$ 


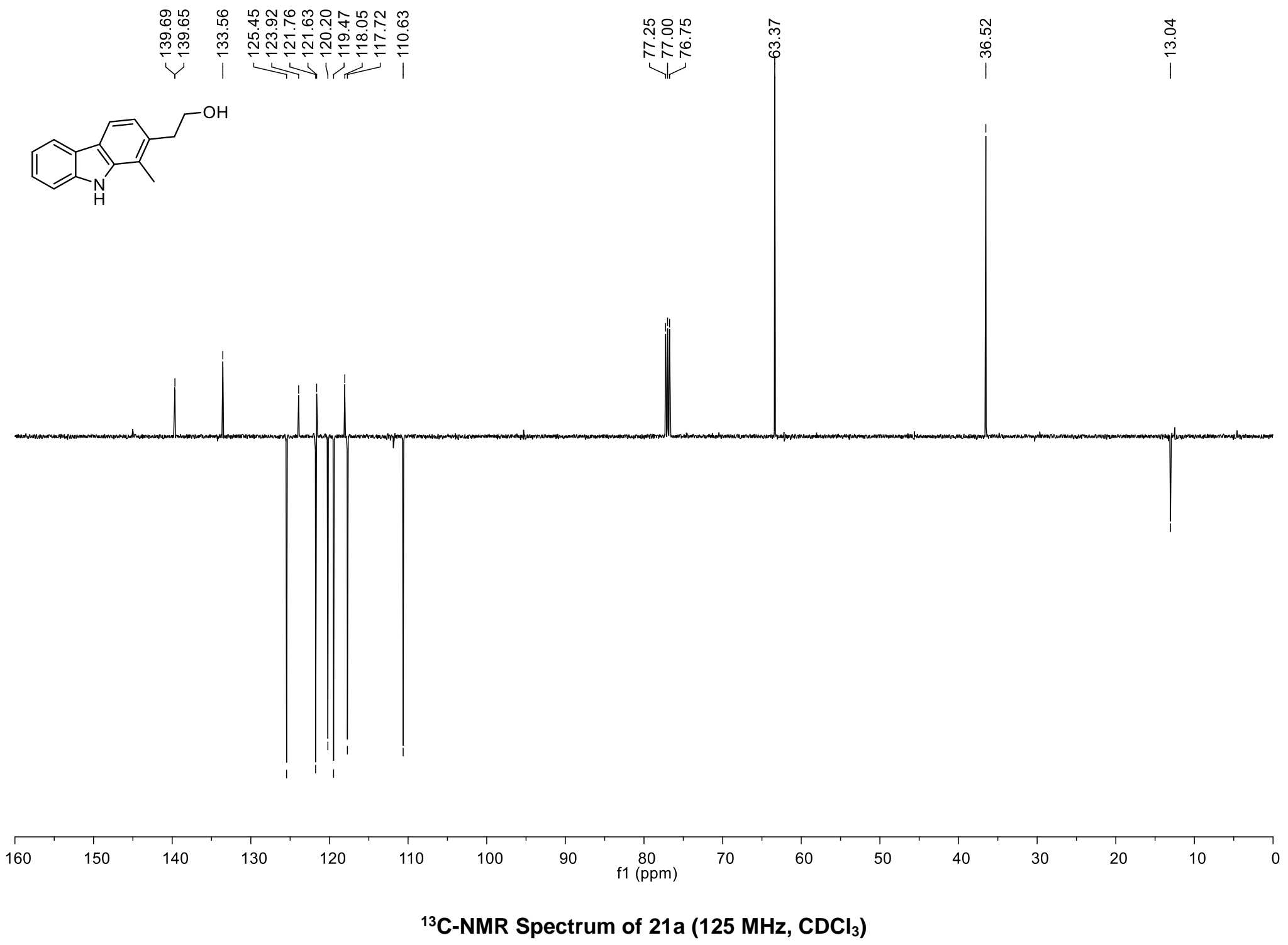



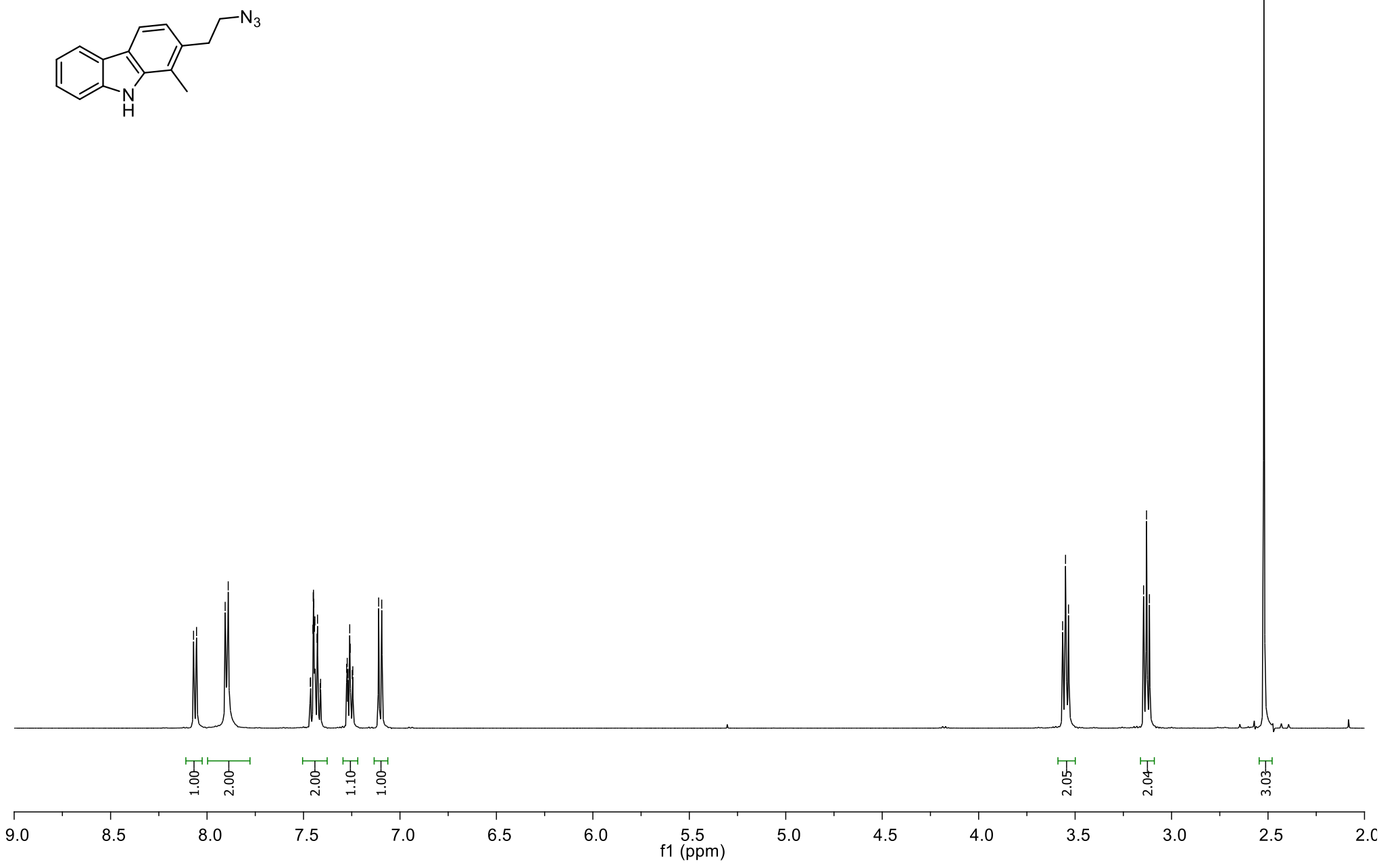

${ }^{1} \mathrm{H}$-NMR Spectrum of $23 \mathrm{a}\left(500 \mathrm{MHz}, \mathrm{CDCl}_{3}\right)$ 


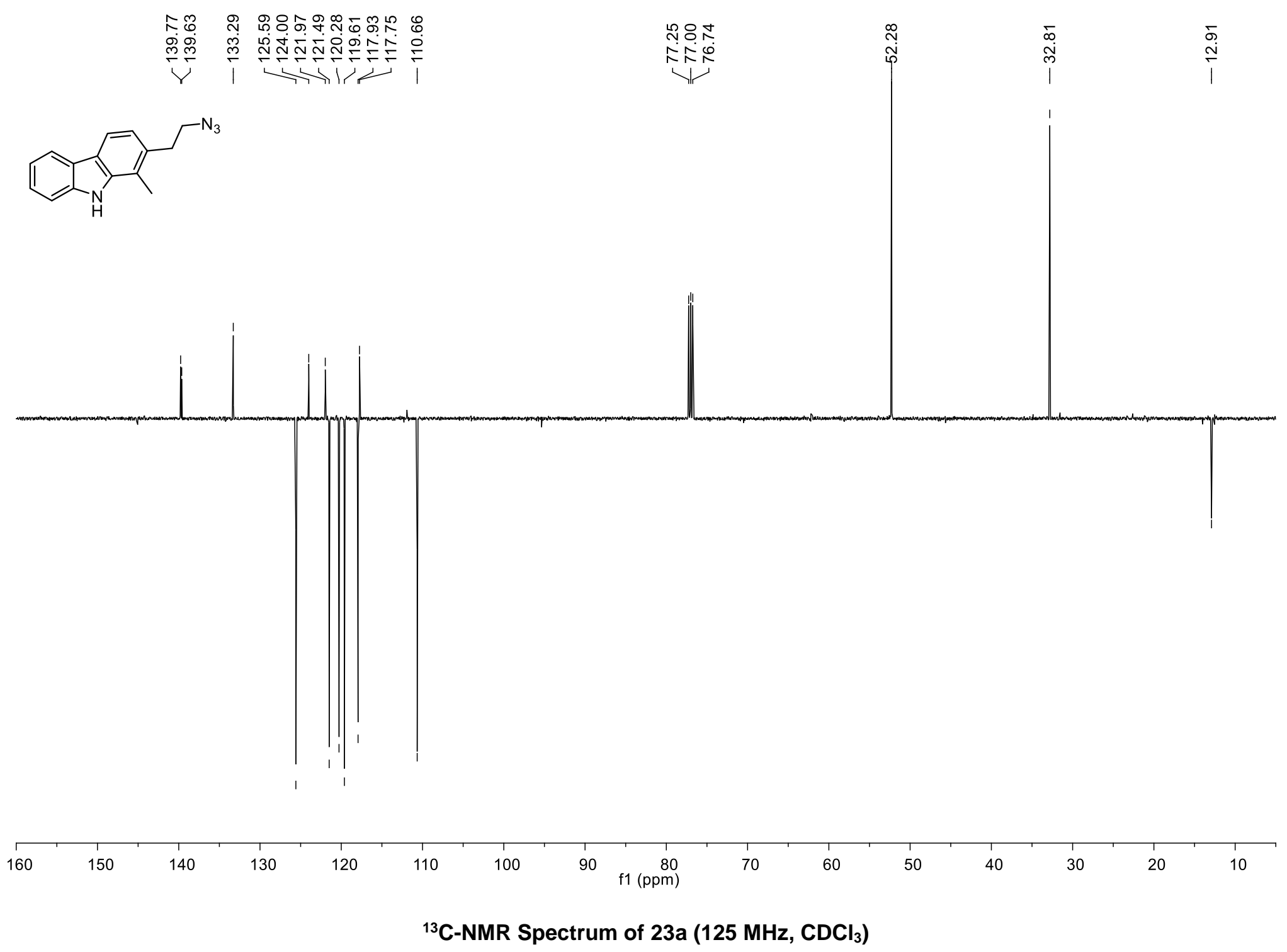




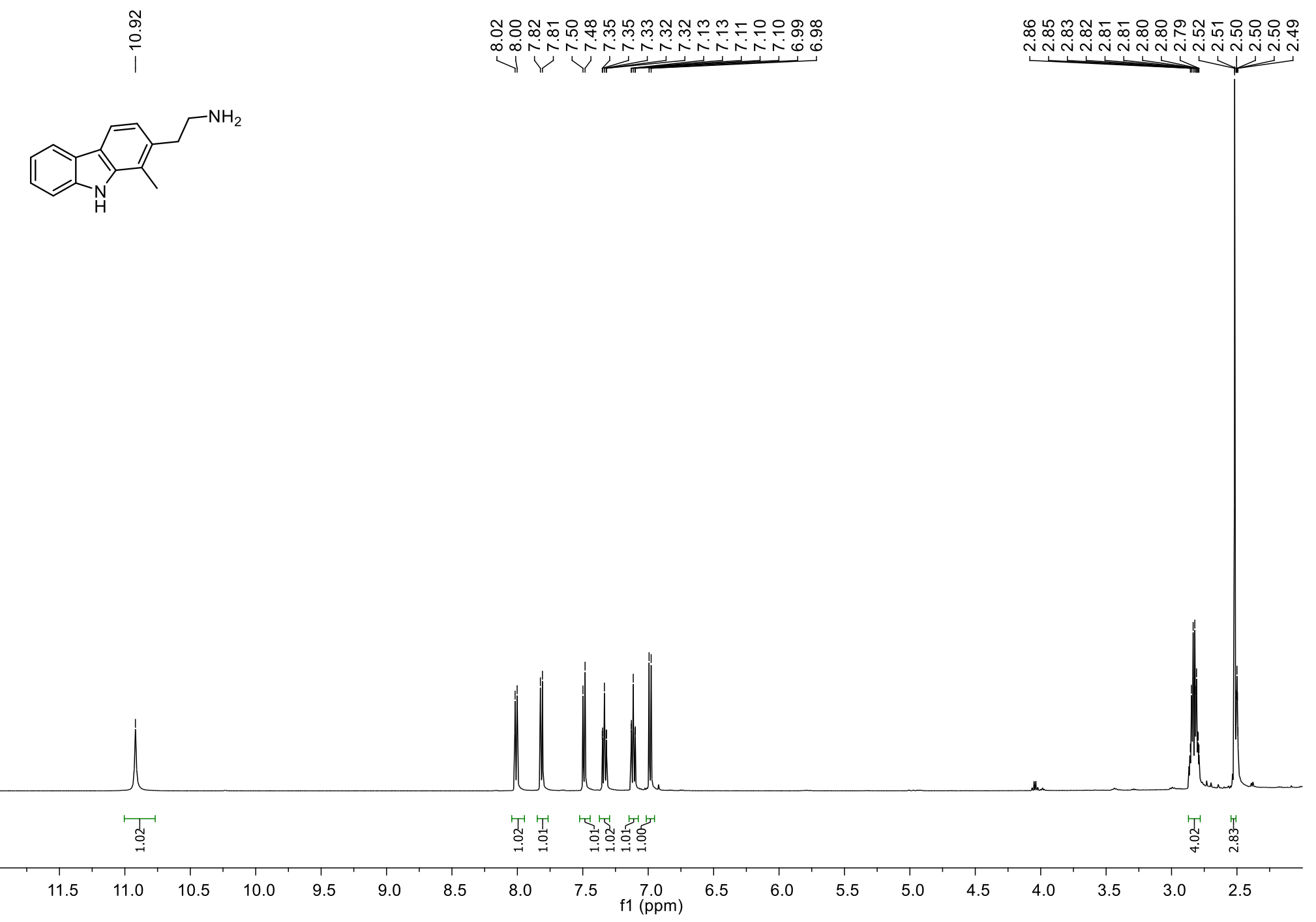

${ }^{1} \mathrm{H}-\mathrm{NMR}$ Spectrum of $24 \mathrm{a}\left(500 \mathrm{MHz}\right.$, DMSO- $\left.d_{6}\right)$ 


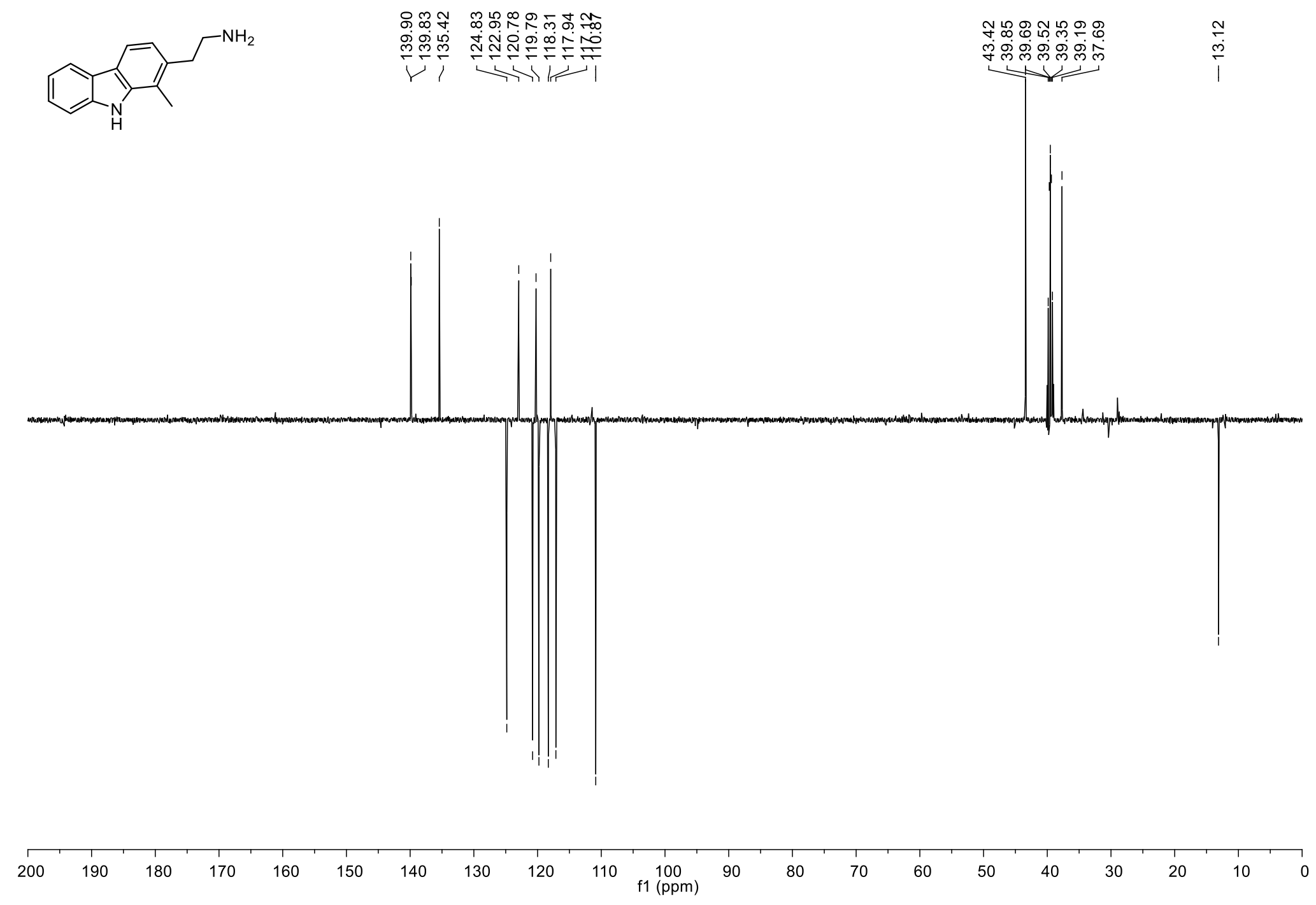

${ }^{13} \mathrm{C}-N M R$ Spectrum of 24a (125 MHz, DMSO- $d_{6}$ ) 


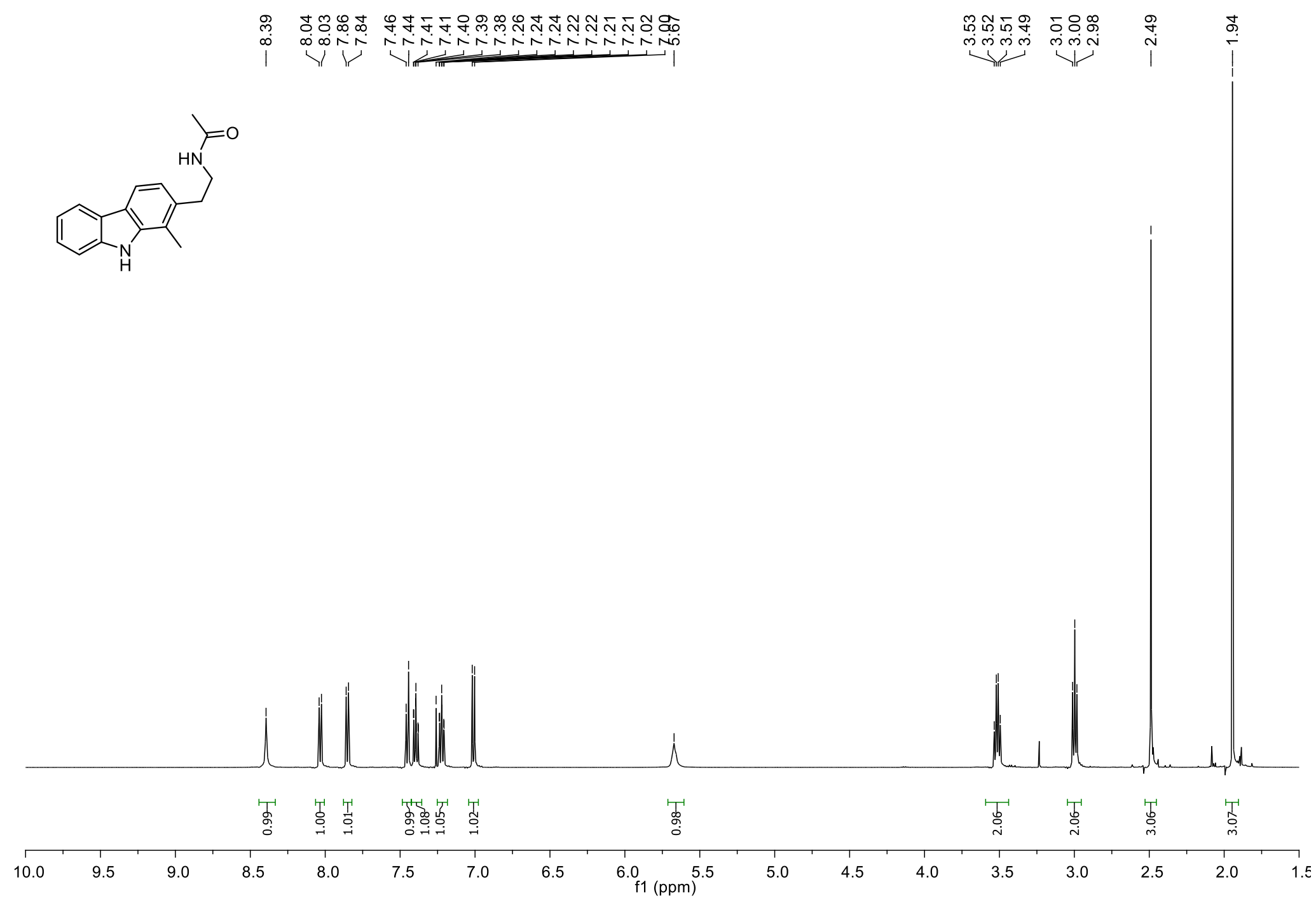

${ }^{1} \mathrm{H}-\mathrm{NMR}$ Spectrum of $25\left(500 \mathrm{MHz}, \mathrm{CDCl}_{3}\right)$ 


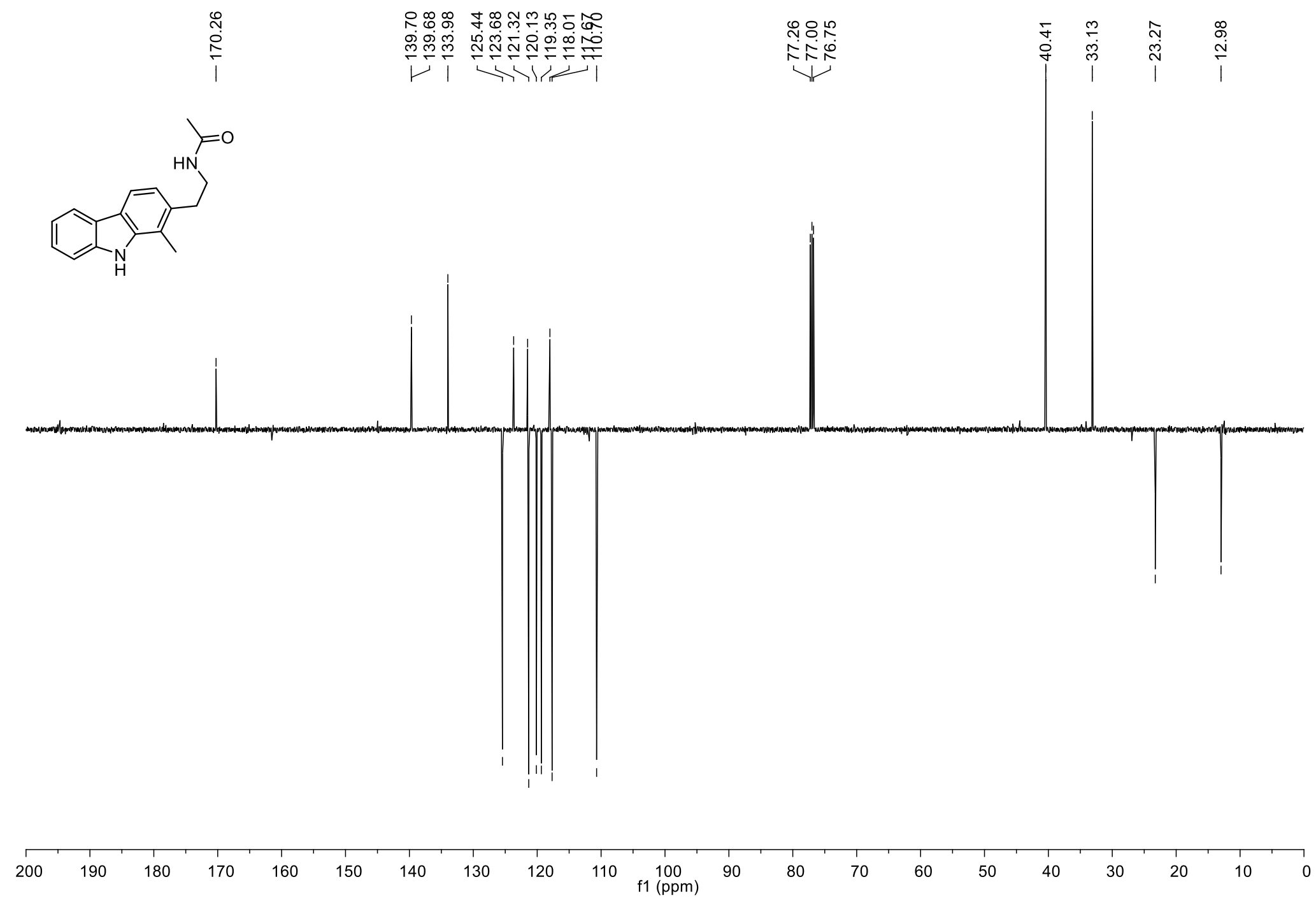

${ }^{13} \mathrm{C}$-NMR Spectrum of $25\left(125 \mathrm{MHz}, \mathrm{CDCl}_{3}\right)$ 


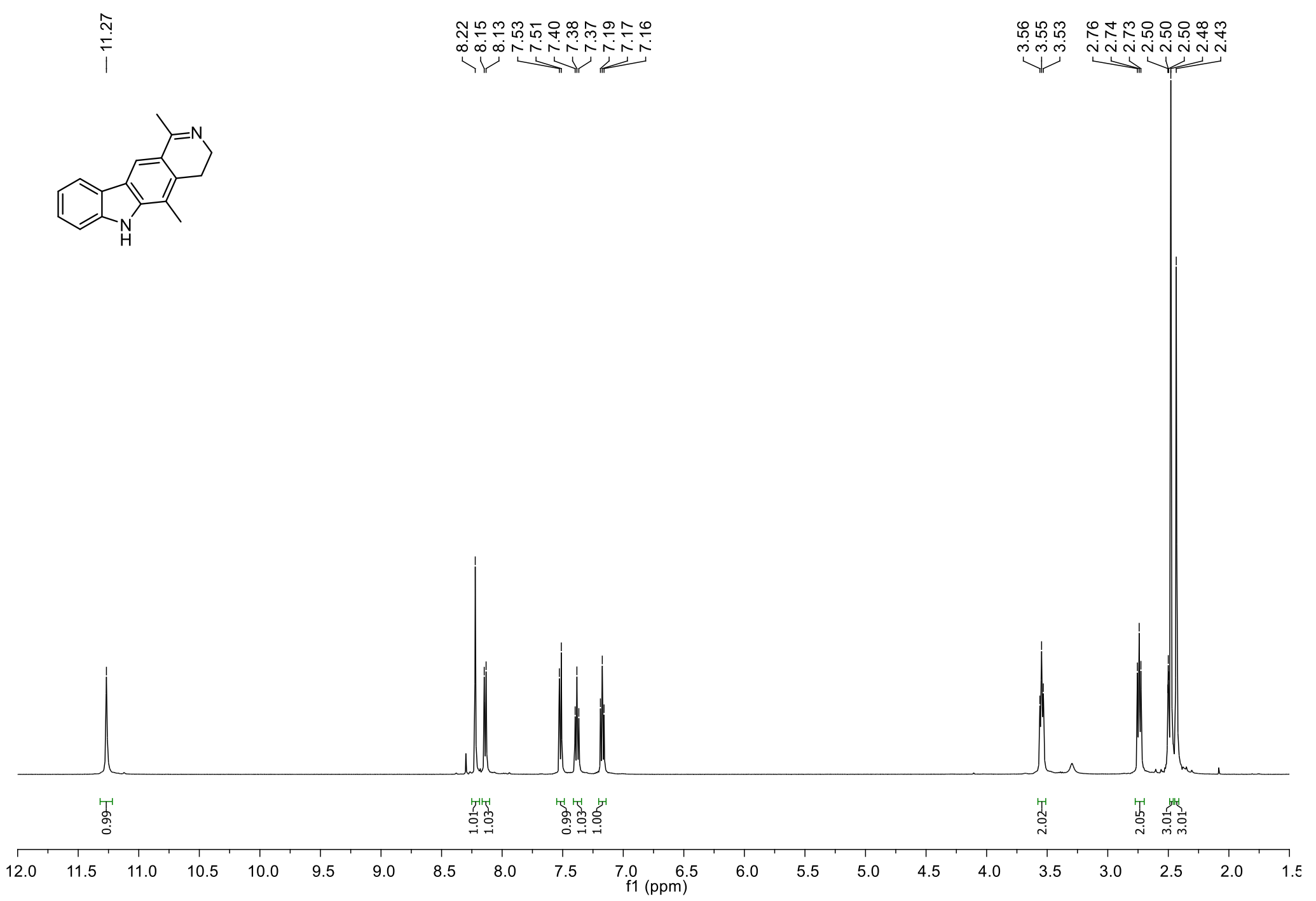

${ }^{1} \mathrm{H}-\mathrm{NMR}$ Spectrum of 26 (500 MHz, DMSO- $d_{6}$ ) 


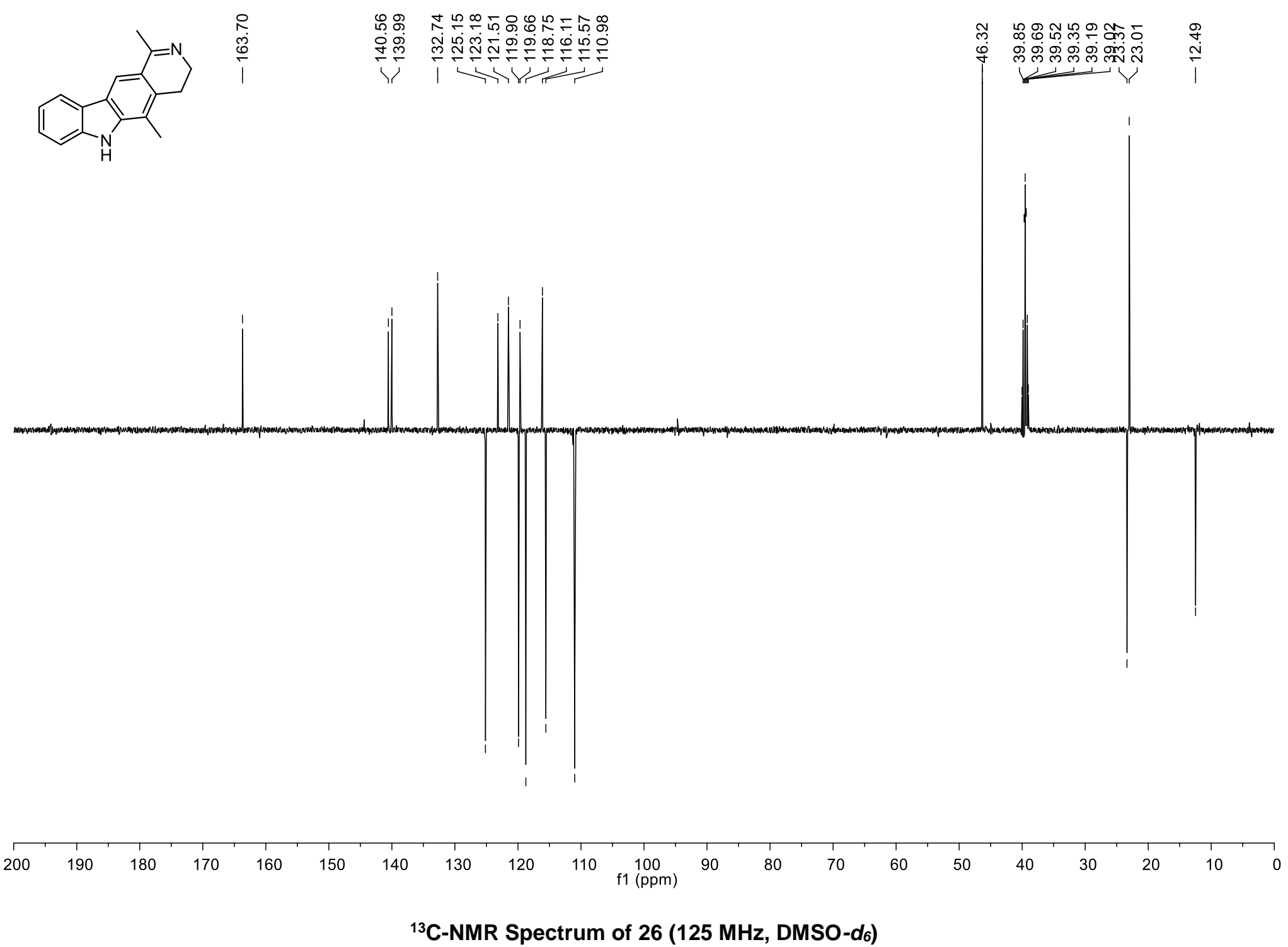




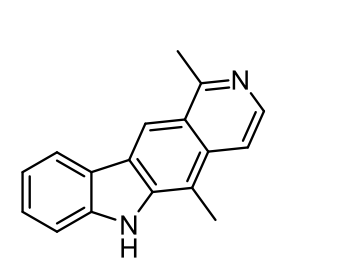

శ.

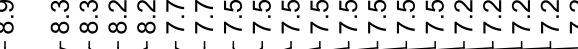

1 पレ

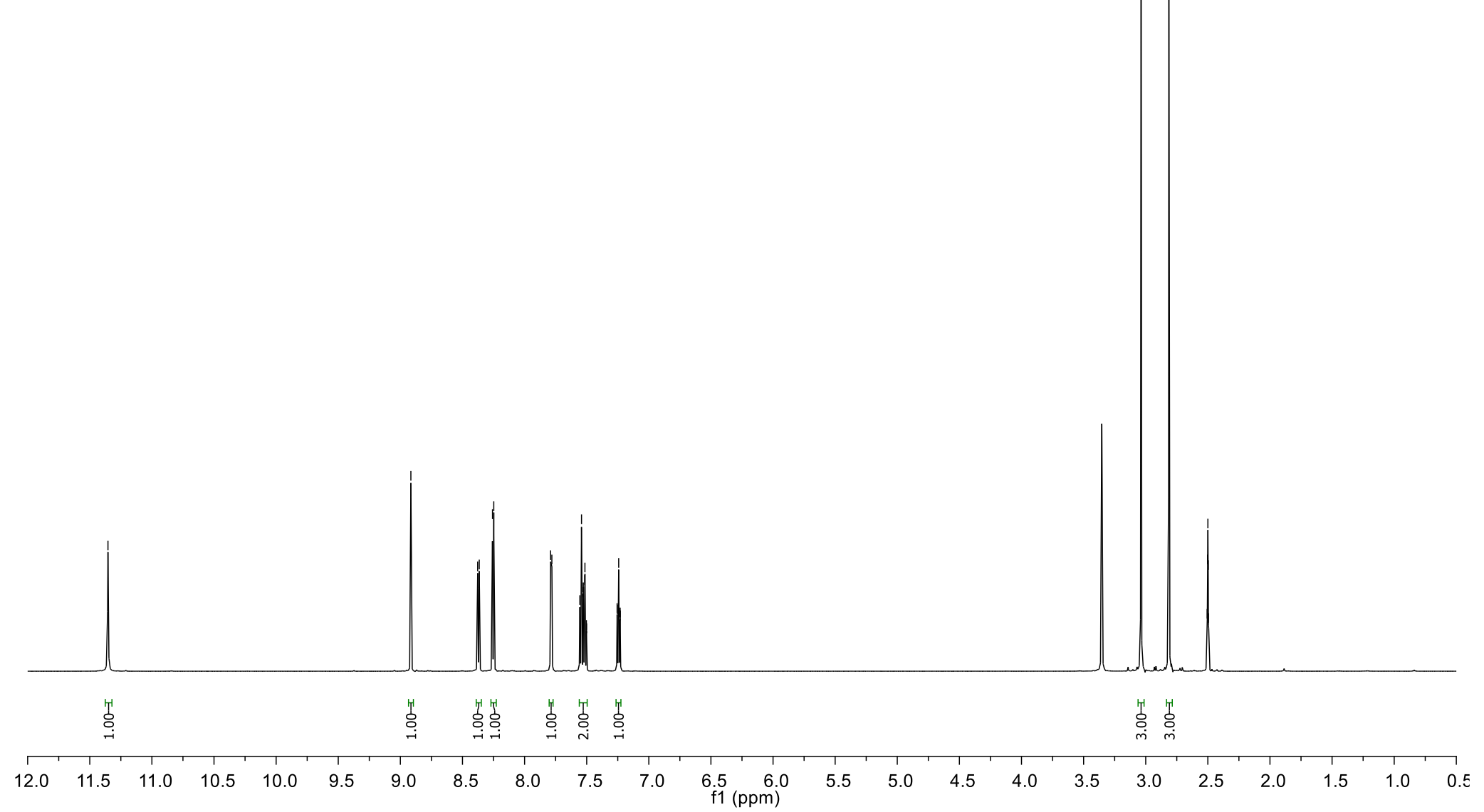

${ }^{1} \mathrm{H}-\mathrm{NMR}$ Spectrum of Olivacine (600 MHz, DMSO- $d_{6}$ ) 

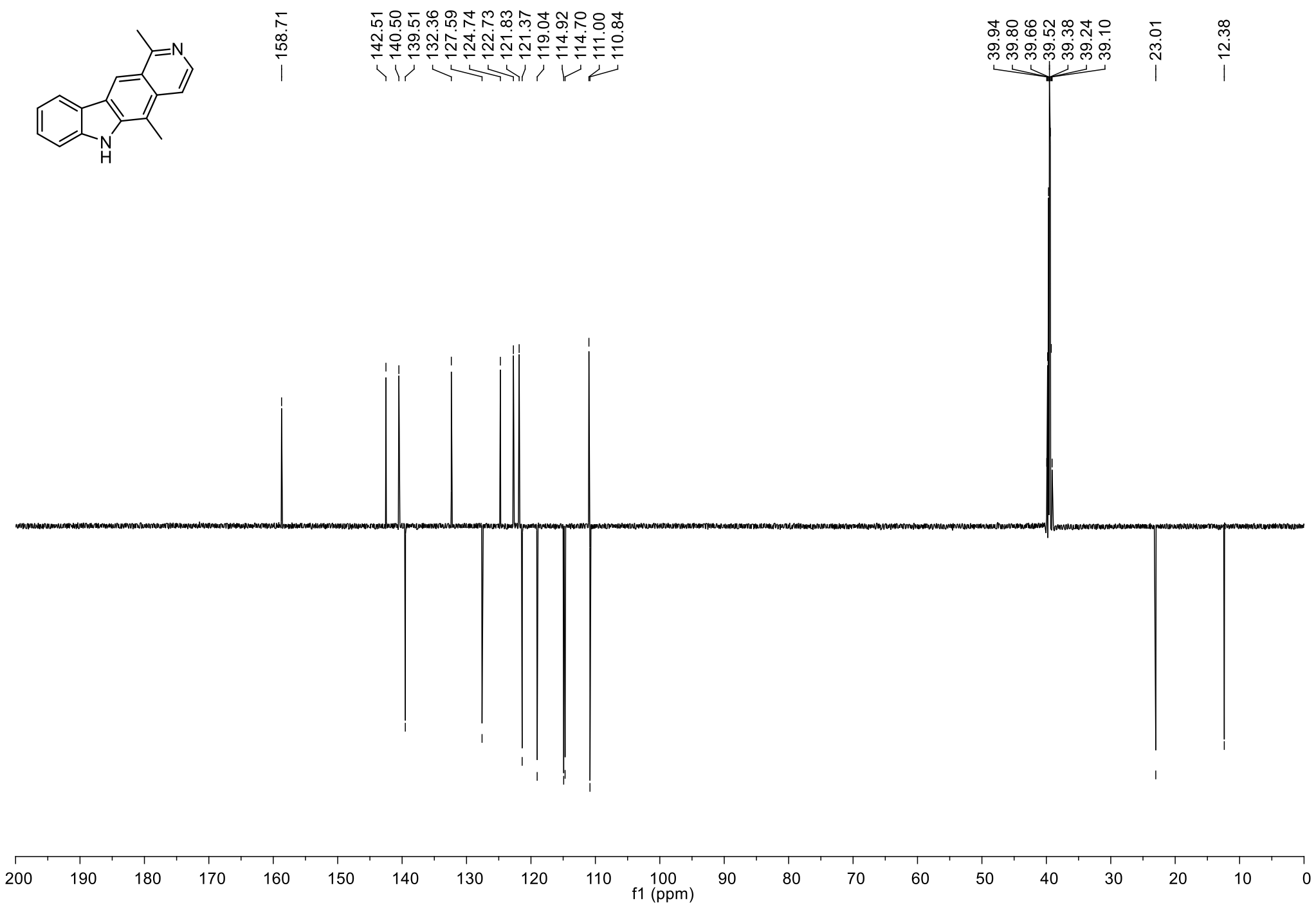

${ }^{13} \mathrm{C}-N M R$ Spectrum of Olivacine $\left(150 \mathrm{MHz}\right.$, DMSO- $\left.d_{6}\right)$

S46 


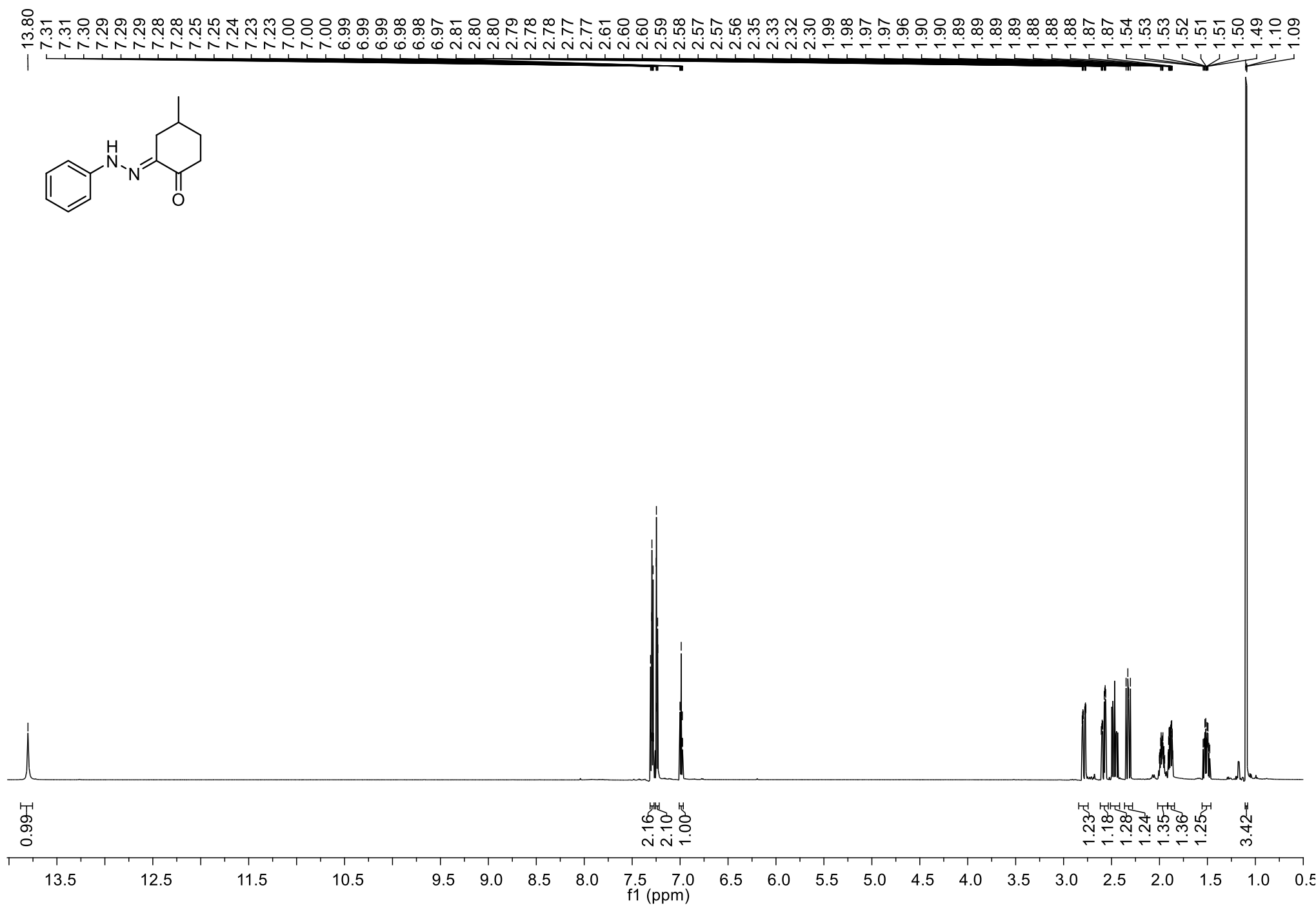

${ }^{1} \mathrm{H}-\mathrm{NMR}$ Spectrum of $2 \mathrm{~b}\left(600 \mathrm{MHz}, \mathrm{CDCl}_{3}\right)$ 


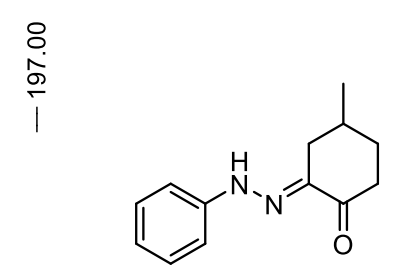

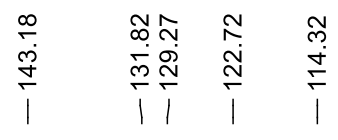

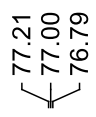
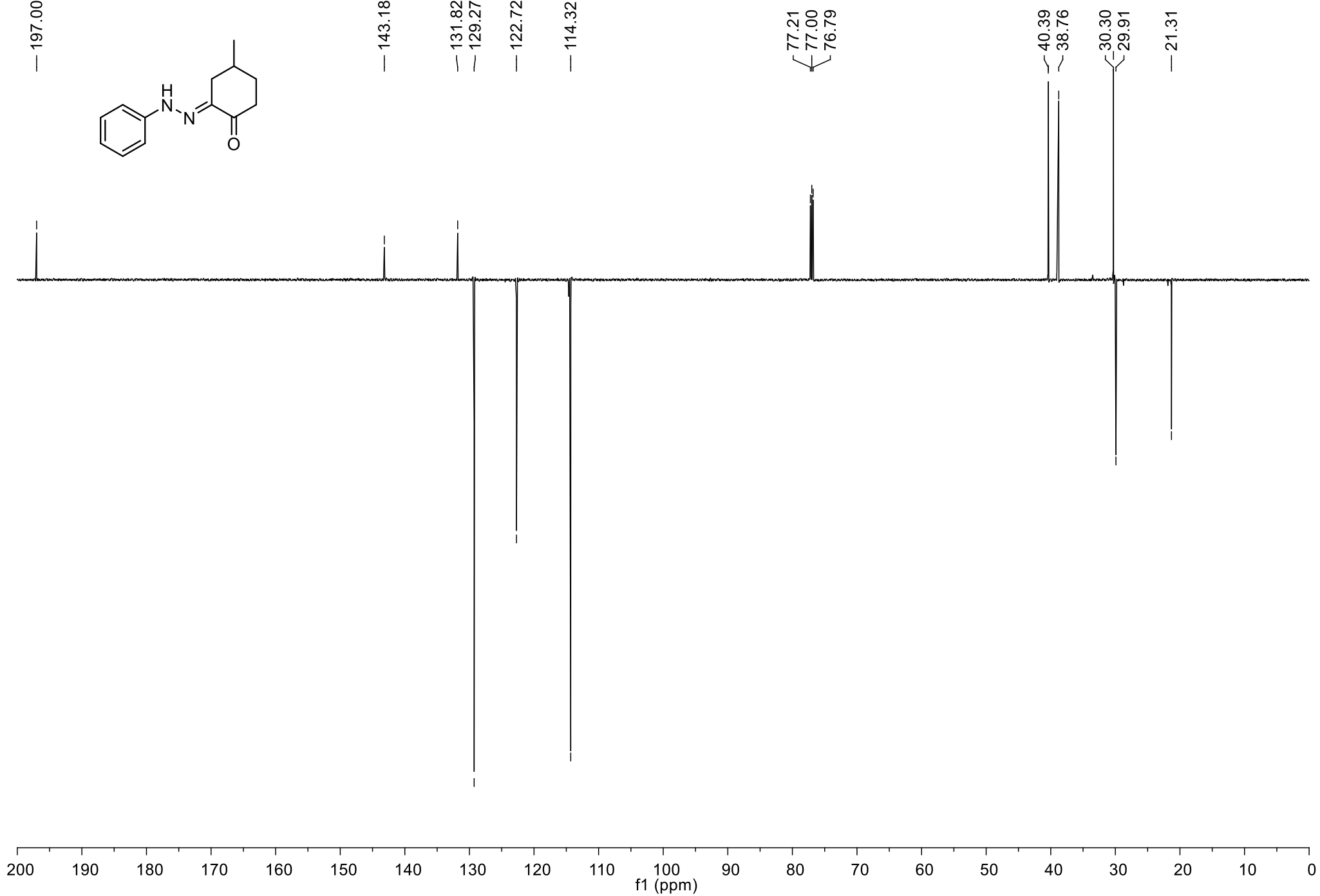

${ }^{13} \mathrm{C}$-NMR Spectrum of $2 \mathrm{~b}\left(150 \mathrm{MHz}, \mathrm{CDCl}_{3}\right)$

S48 


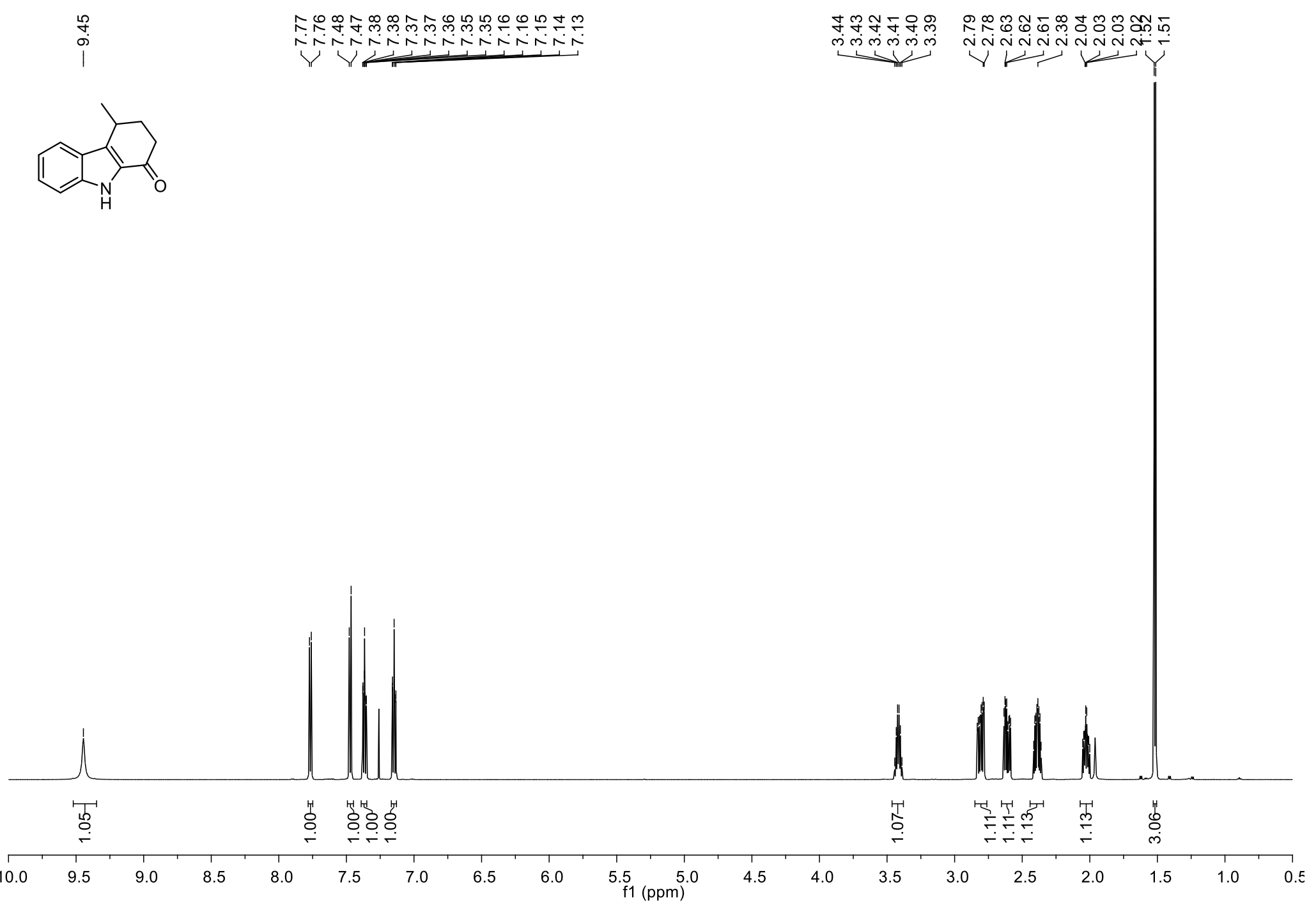

${ }^{1} \mathrm{H}-\mathrm{NMR}$ Spectrum of $3 \mathrm{~b}\left(600 \mathrm{MHz}, \mathrm{CDCl}_{3}\right)$ 


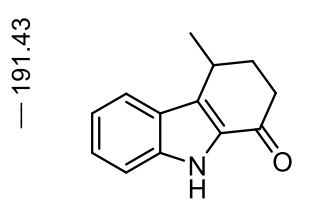

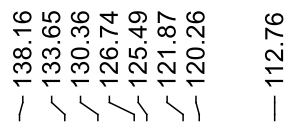

梅

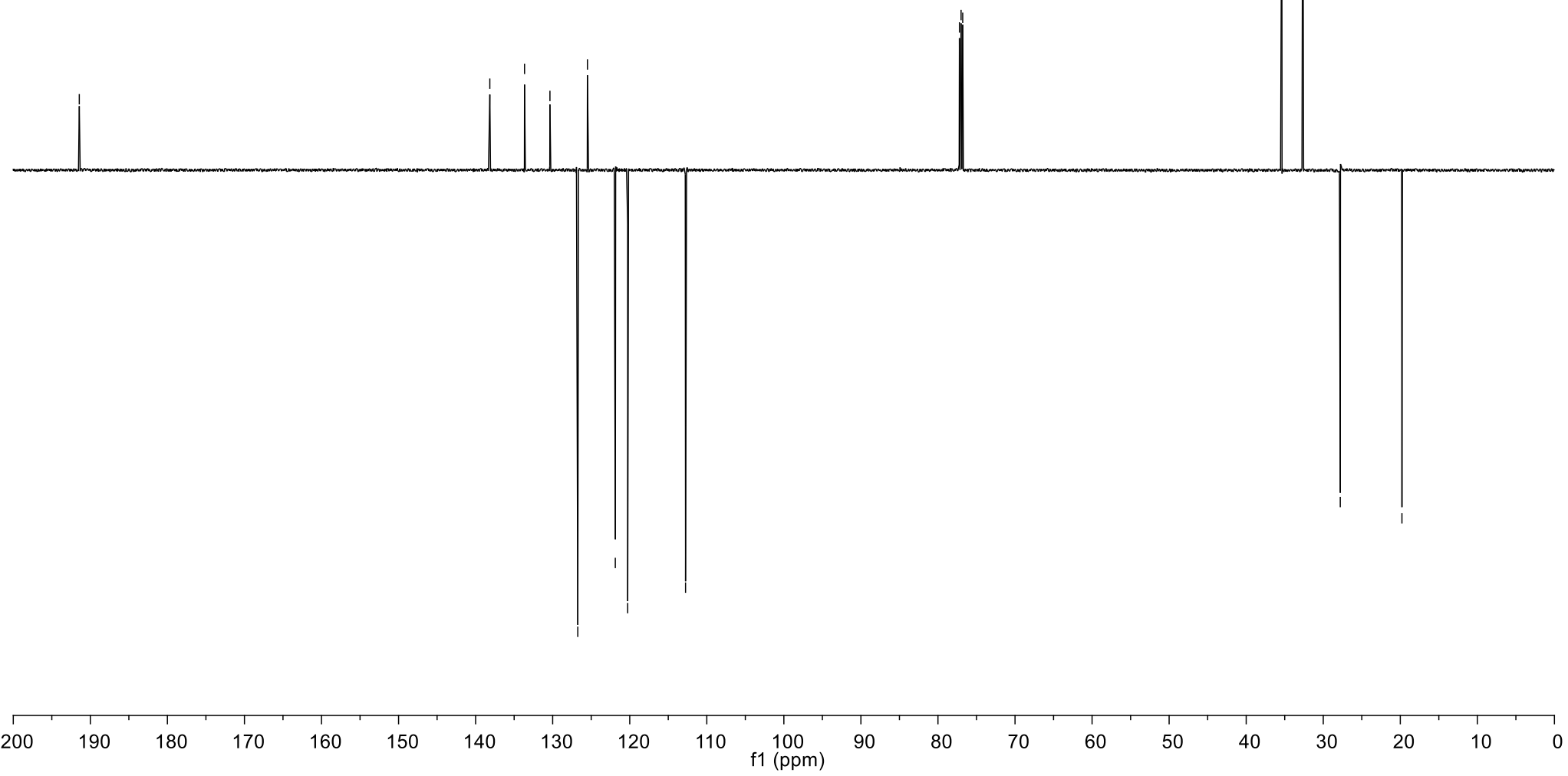

${ }^{13} \mathrm{C}$-NMR Spectrum of $3 \mathrm{~b}\left(150 \mathrm{MHz}, \mathrm{CDCl}_{3}\right)$

$\mathrm{S} 50$ 
Table S2. NMR spectral data of compound $\mathbf{5 b}$.

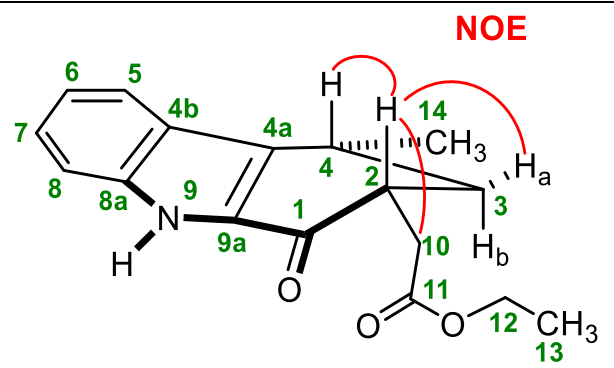

$5 b$

\begin{tabular}{|c|c|c|c|c|c|}
\hline $\mathrm{C \#}$ & $\delta_{\mathrm{C}}$ & $\delta_{\mathrm{H}}$ & Mult. & COSY & NOE-DIFF \\
\hline 1 & 192.0 & $\mathrm{C}=\mathrm{O}$ & $\mathrm{C}$ & & \\
\hline 2 & 43.7 & $3.22-3.17(\mathrm{~m}, 1 \mathrm{H}, H-2)$ & $\mathrm{CH}$ & $2 \times H-10, H_{a}-3, H_{b}-3$ & $\begin{array}{l}H-10, \quad H-4, \\
H_{a}-3\end{array}$ \\
\hline 3 & 41.0 & $2.30\left(\mathrm{td}, J=12.9,4.3 \mathrm{~Hz}, 1 \mathrm{H}, H_{a^{-}}-3\right)$ & $\mathrm{CH}_{2}$ & $H_{a^{-}}-3, H-2, H-4$ & \\
\hline 3 & - & $1.84\left(\mathrm{~m}, 1 \mathrm{H}, H_{b^{-}} 3\right)$ & - & $H_{a}-3, H-2, H-4$ & \\
\hline 4 & 30.1 & $3.46-3.41(\mathrm{~m}, 1 \mathrm{H}, H-4)$ & $\mathrm{CH}$ & $H-14, H_{a}-3, H_{b}-3$ & \\
\hline $4 \mathbf{a}$ & 125.7 & & $\mathrm{C}$ & & \\
\hline 4b & 130.4 & & $\mathrm{C}$ & & \\
\hline 5 & 120.4 & $7.13(\mathrm{t}, J=7.5 \mathrm{~Hz}, 1 \mathrm{H}, H-5)$ & $\mathrm{CH}$ & & \\
\hline 6 & 126.7 & $7.36(\mathrm{t}, J=7.8 \mathrm{~Hz}, 1 \mathrm{H}, H-6)$ & $\mathrm{CH}$ & & \\
\hline 7 & 122.8 & $7.87(\mathrm{~d}, J=8.2 \mathrm{~Hz}, 1 \mathrm{H}, H-7)$ & $\mathrm{CH}$ & & \\
\hline 8 & 113.2 & $7.49(\mathrm{dd}, J=11.2,3.6 \mathrm{~Hz}, 1 \mathrm{H}, H-8)$ & $\mathrm{CH}$ & & \\
\hline \multirow[t]{2}{*}{$\mathbf{8 a}$} & 132.9 & $C-8 \mathrm{a}$ or $C-9 \mathrm{a}$ & $\mathrm{C}$ & & \\
\hline & & $9.70(\mathrm{~s}, \mathrm{~N}-H)$ & - & & \\
\hline $9 a$ & 138.8 & $C-8 \mathrm{a}$ or $C-9 \mathrm{a}$ & $\mathrm{C}$ & & \\
\hline 10 & 34.8 & $3.10(\mathrm{dd}, J=16.4,5.5 \mathrm{~Hz}, 1 \mathrm{H}, H-10)$ & $\mathrm{CH}_{2}$ & $H-2, H-10$ & \\
\hline 10 & - & $2.44(\mathrm{dd}, J=16.4,7.2 \mathrm{~Hz}, 1 \mathrm{H}, H-10)$ & - & $H-2, H-10$ & \\
\hline 11 & 172.8 & $\mathrm{C}=\mathrm{O}$ & $\mathrm{C}$ & & \\
\hline 12 & 60.8 & $4.22(\mathrm{q}, J=7.1 \mathrm{~Hz}, 2 \mathrm{H}, H-12)$ & $\mathrm{CH}_{2}$ & $H-13$ & \\
\hline 13 & 14.4 & $1.30(\mathrm{t}, J=7.1 \mathrm{~Hz}, 3 \mathrm{H}, H-13)$ & $\mathrm{CH}_{3}$ & $H-12$ & \\
\hline 14 & 20.9 & $1.61(\mathrm{~d}, J=6.8 \mathrm{~Hz}, 3 \mathrm{H}, H-14)$ & $\mathrm{CH}_{3}$ & $H-4$ & \\
\hline
\end{tabular}




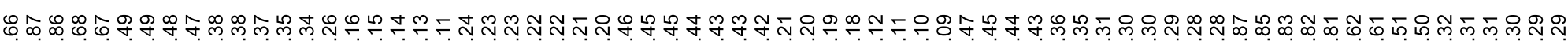

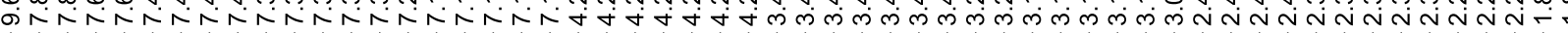

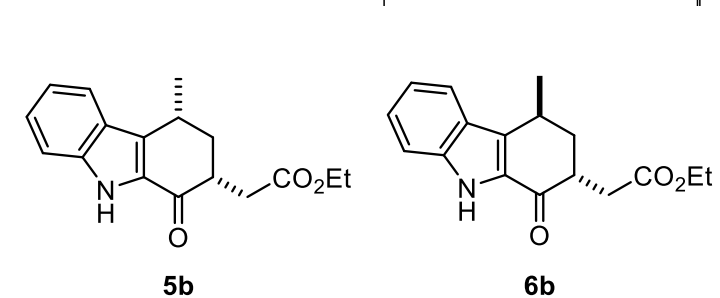

$5 \mathbf{b} / 6 \mathbf{b}=80: 20$
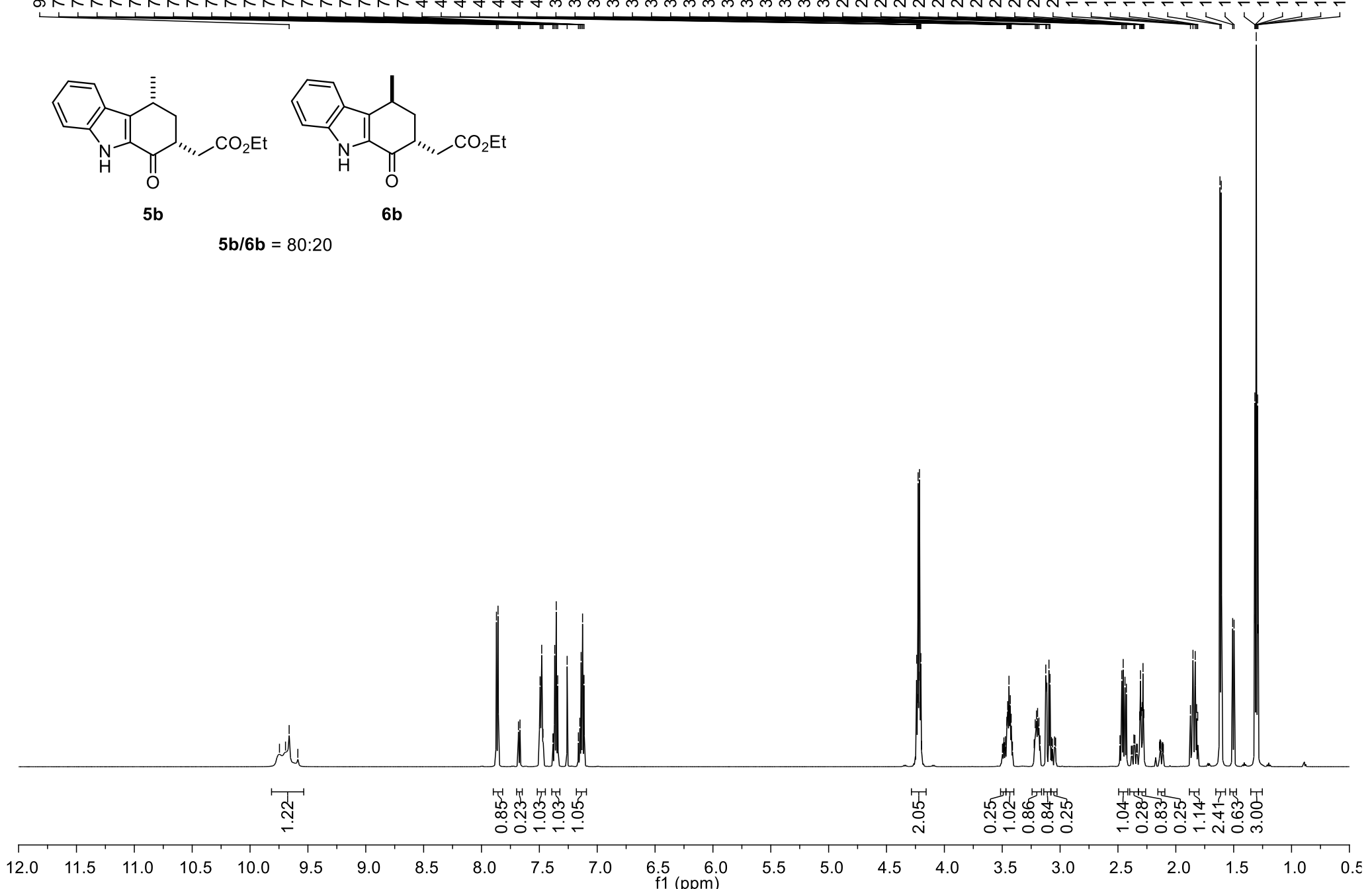

${ }^{1} \mathrm{H}-\mathrm{NMR}$ Spectrum of $5 \mathrm{~b} / 6 \mathrm{~b}\left(600 \mathrm{MHz}^{\mathrm{CDCl}} \mathrm{CDC}_{3}\right)$ 


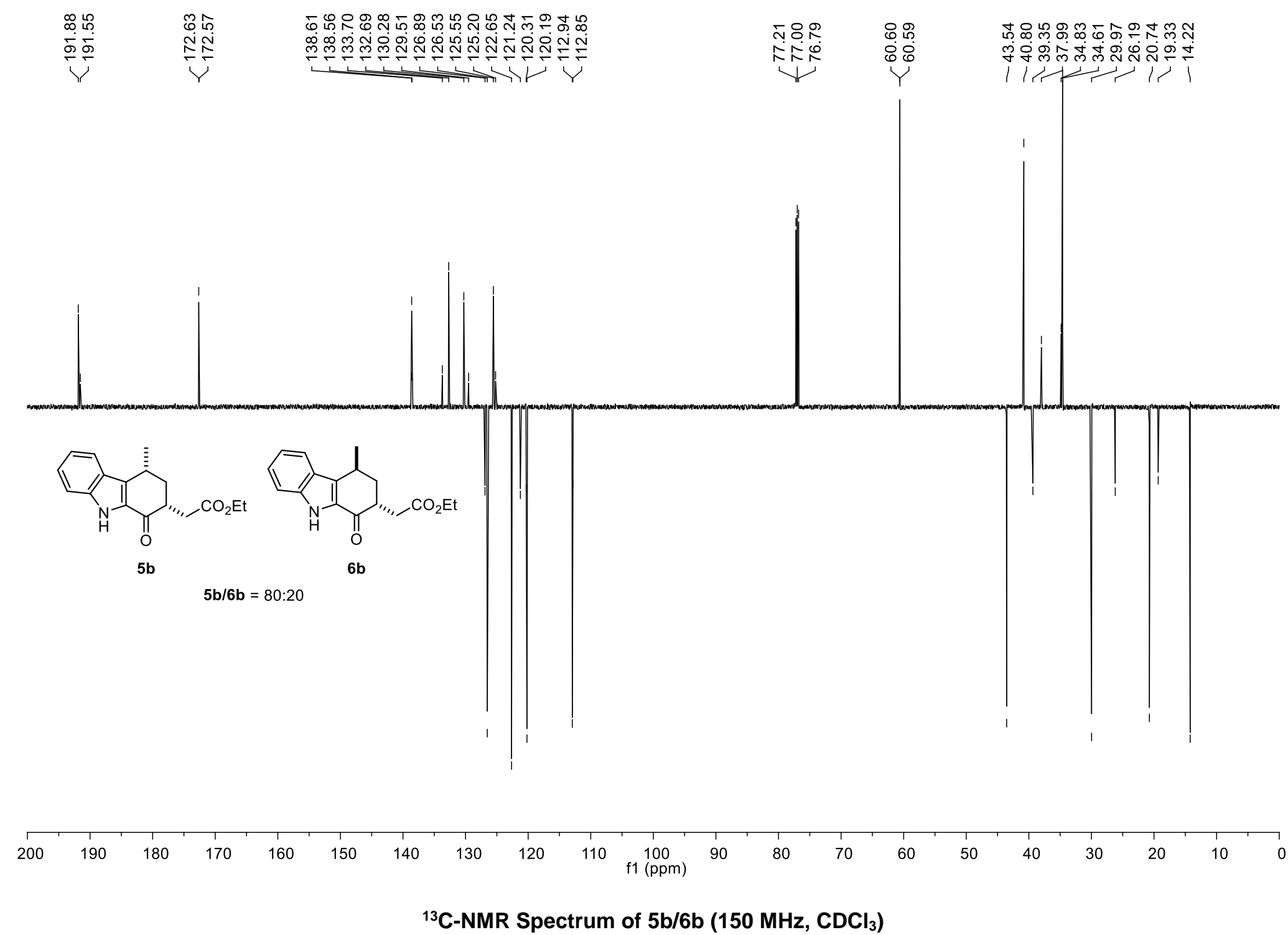




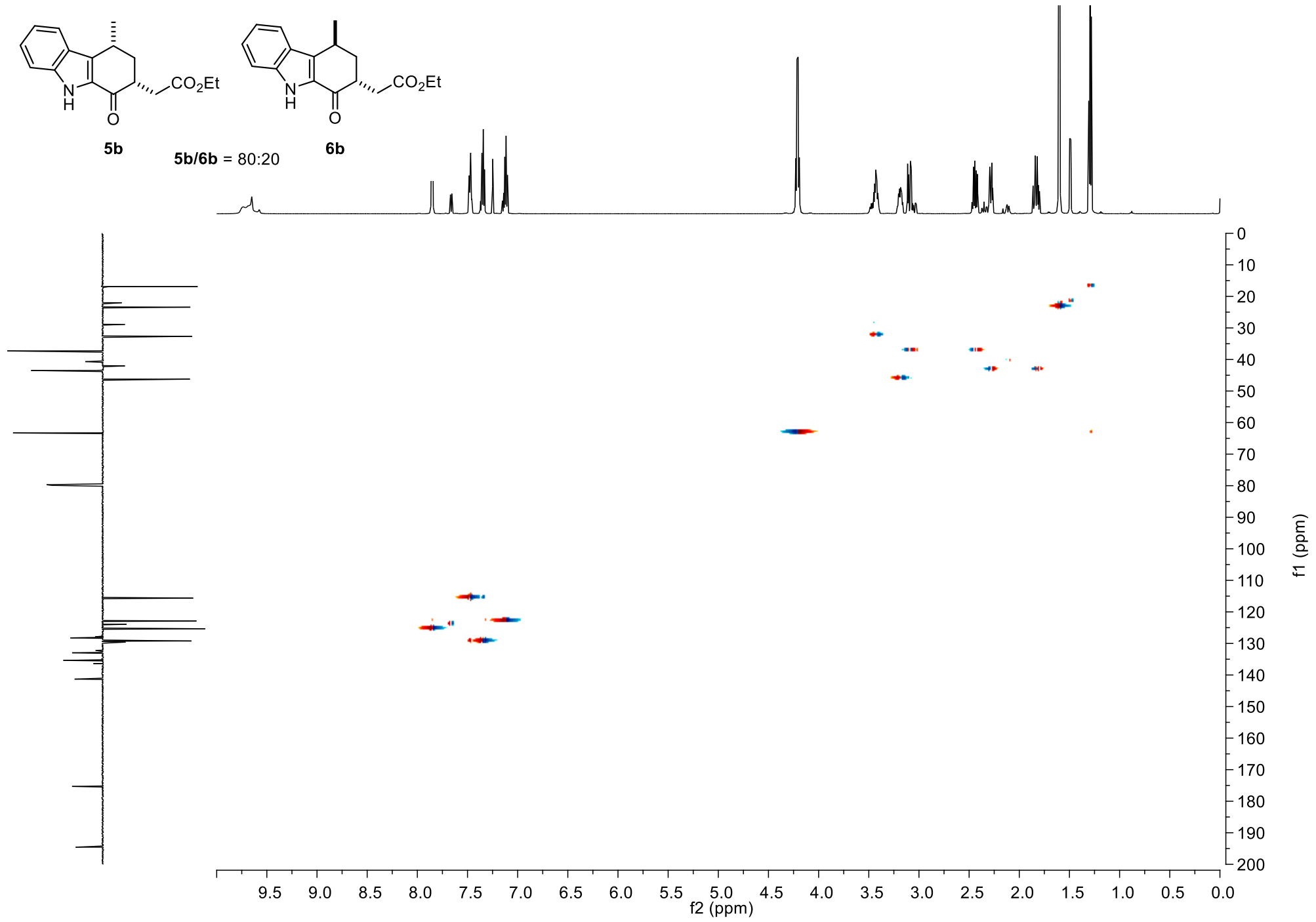

HSQC Spectrum of $5 b / 6 b$ 


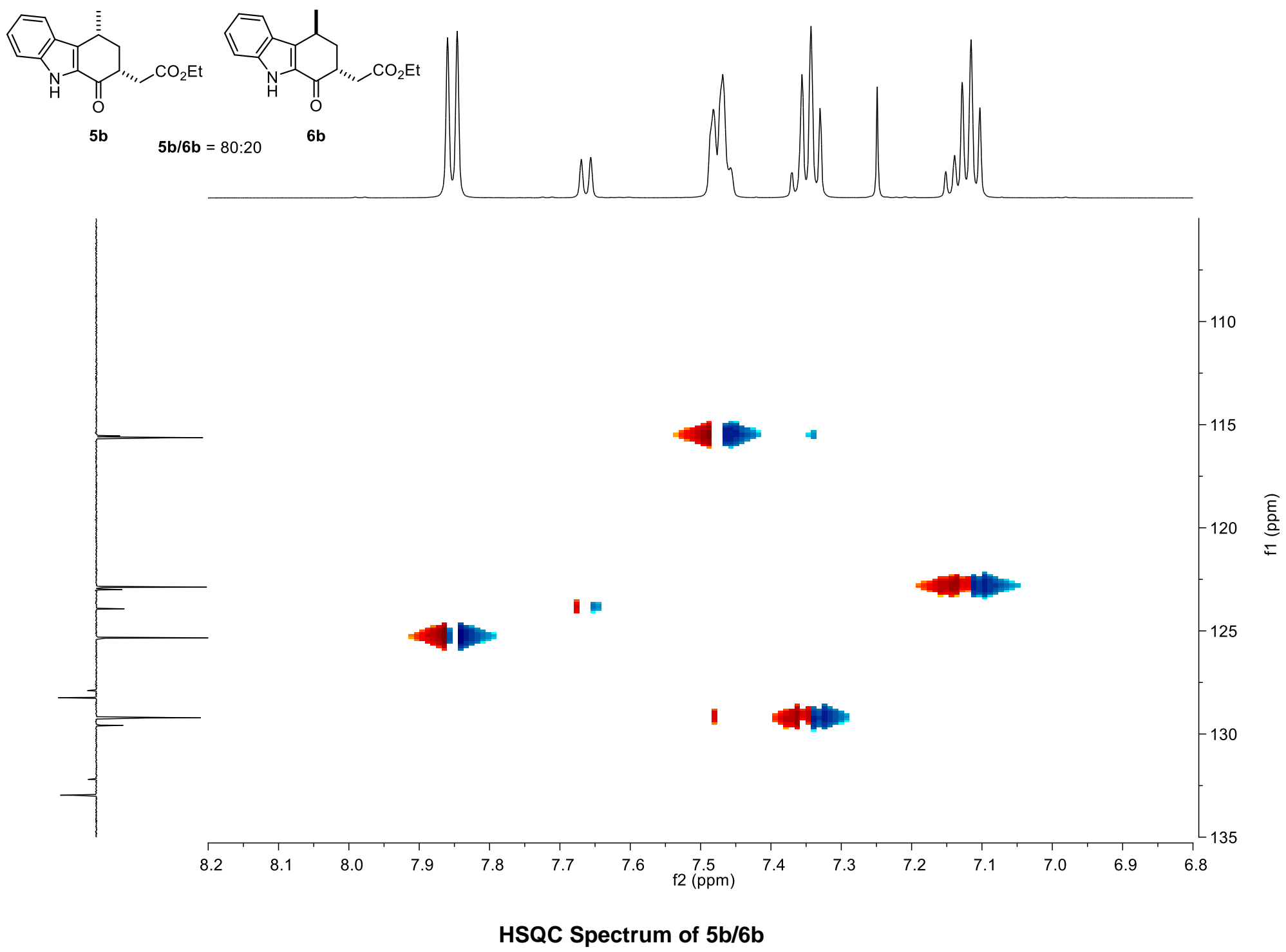

S55 


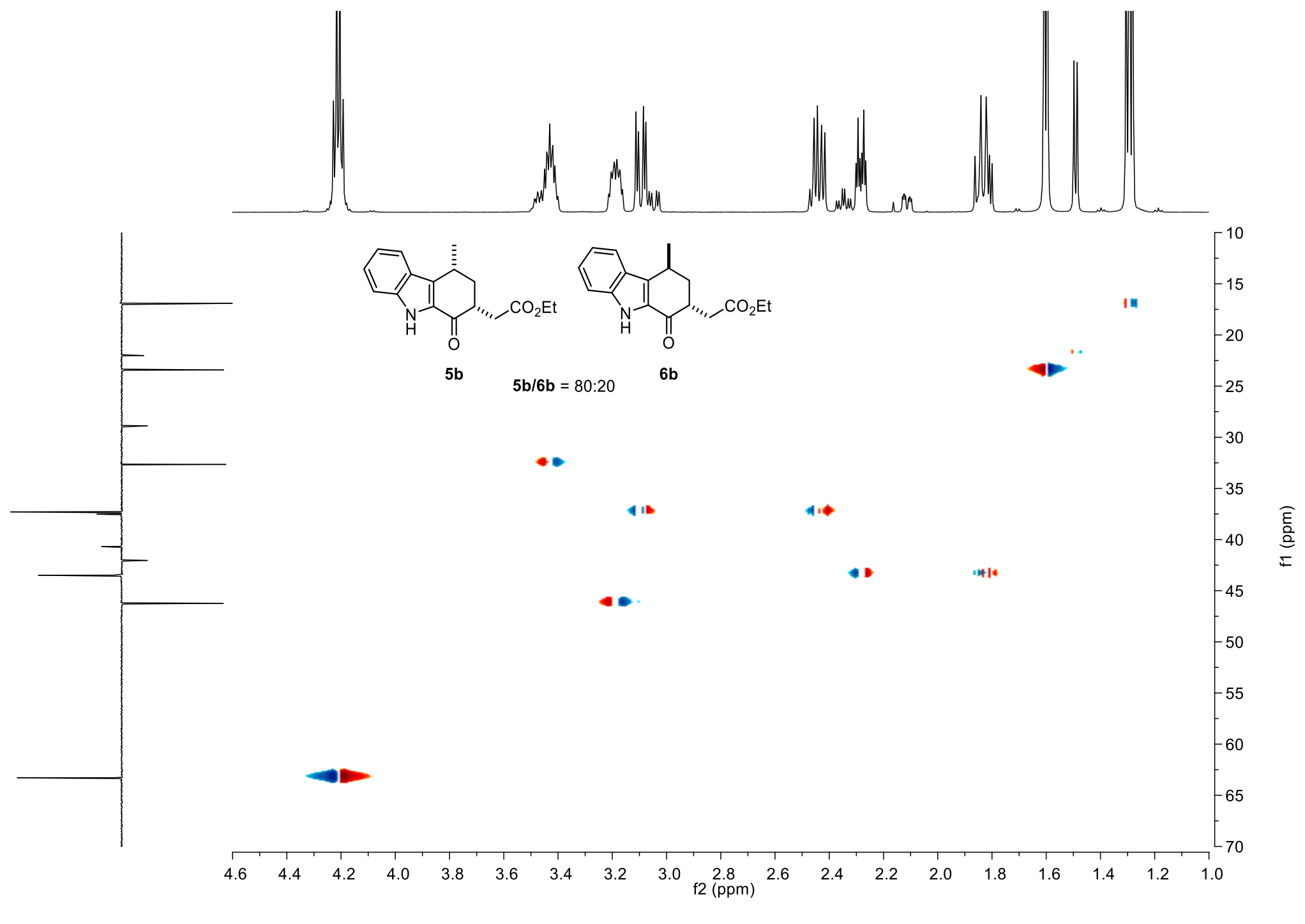

HSQC Spectrum of $5 b / 6 b$ 


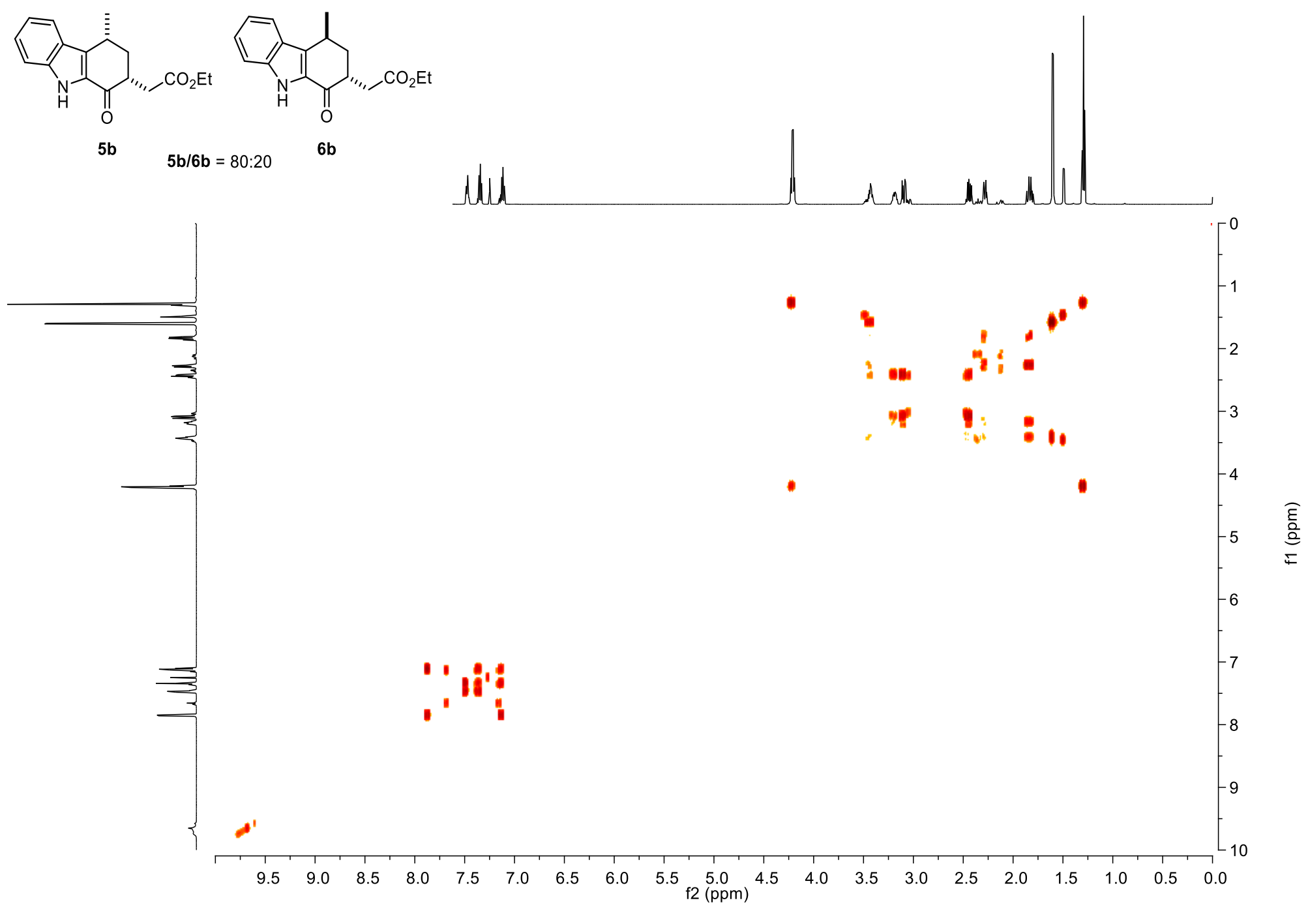

COSY Spectrum of $5 \mathrm{~b} / 6 \mathrm{~b}\left(600 \mathrm{MHz}, \mathrm{CDCl}_{3}\right)$ 


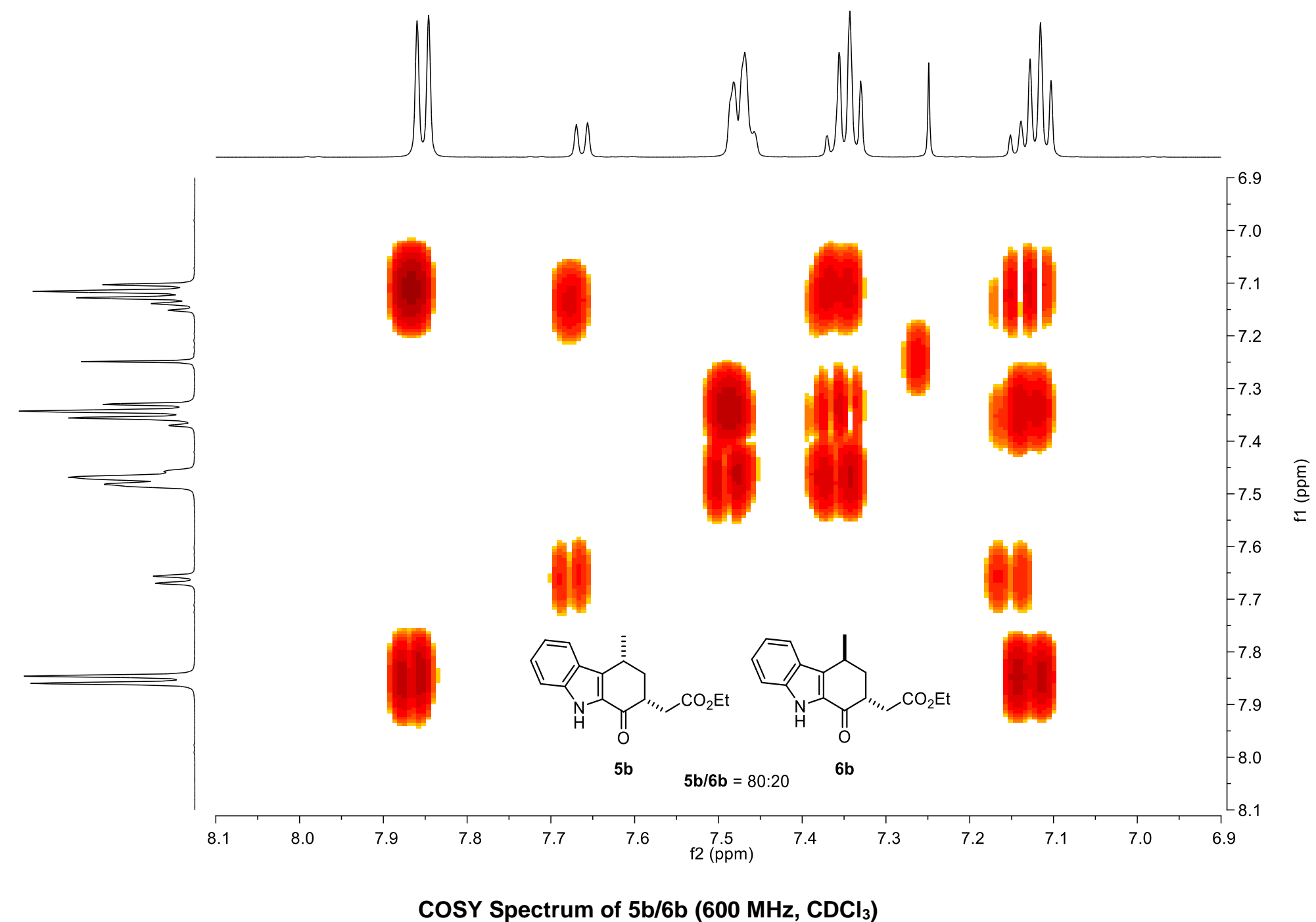




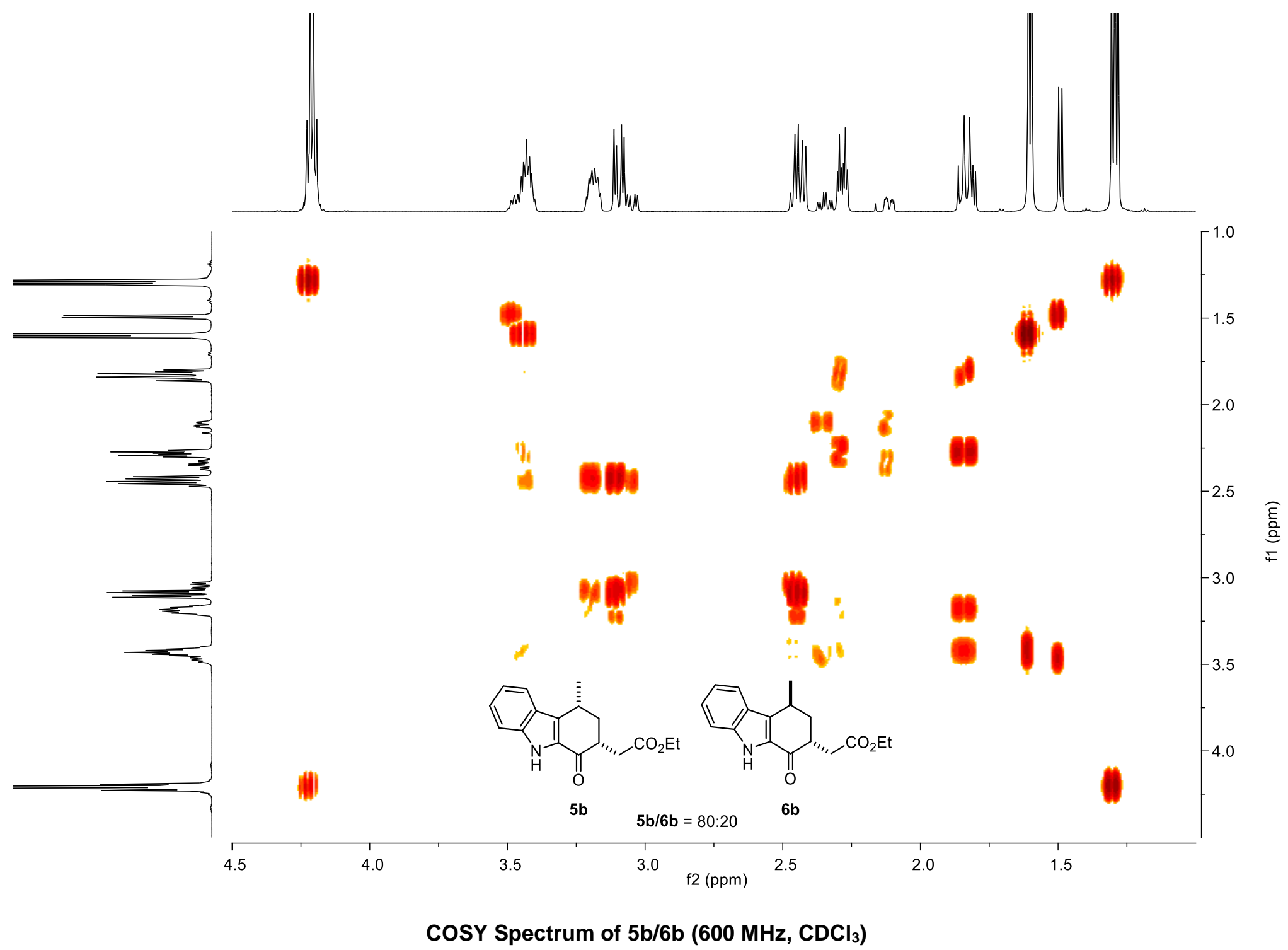




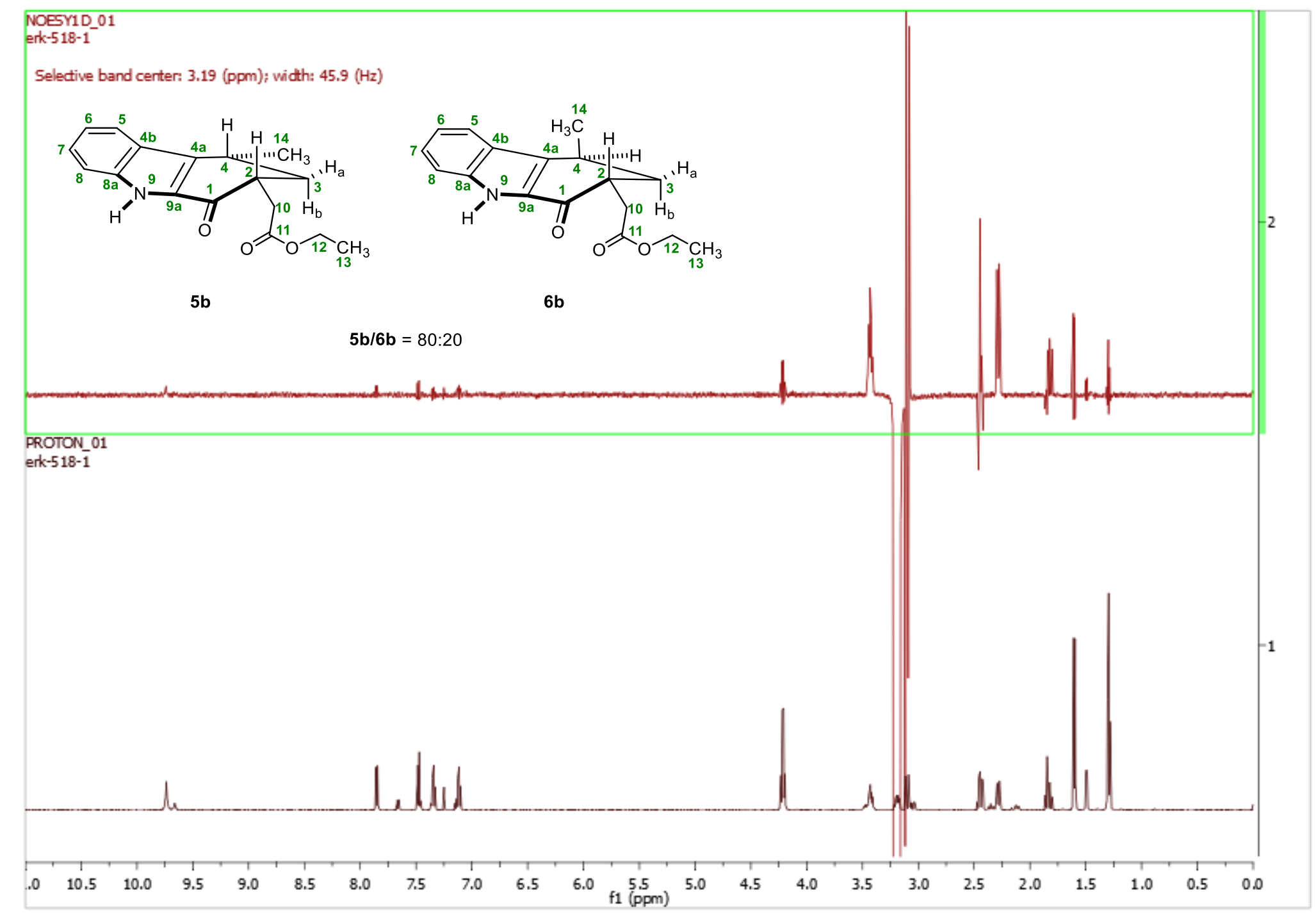

NOE-DIFF Spectrum of $5 \mathrm{~b} / \mathbf{6 b}\left(\mathbf{6 0 0} \mathrm{MHz}, \mathrm{CDCl}_{3}\right)$ : Enhancement of $\boldsymbol{H - 4}, \boldsymbol{H}_{\mathbf{a}}-\mathbf{3}$ and $\boldsymbol{H} \mathbf{1 0}$ signals of $\mathbf{5 b}$ after irradiation of $\boldsymbol{H}-\mathbf{2}$ signal at 3.19 ppm. 


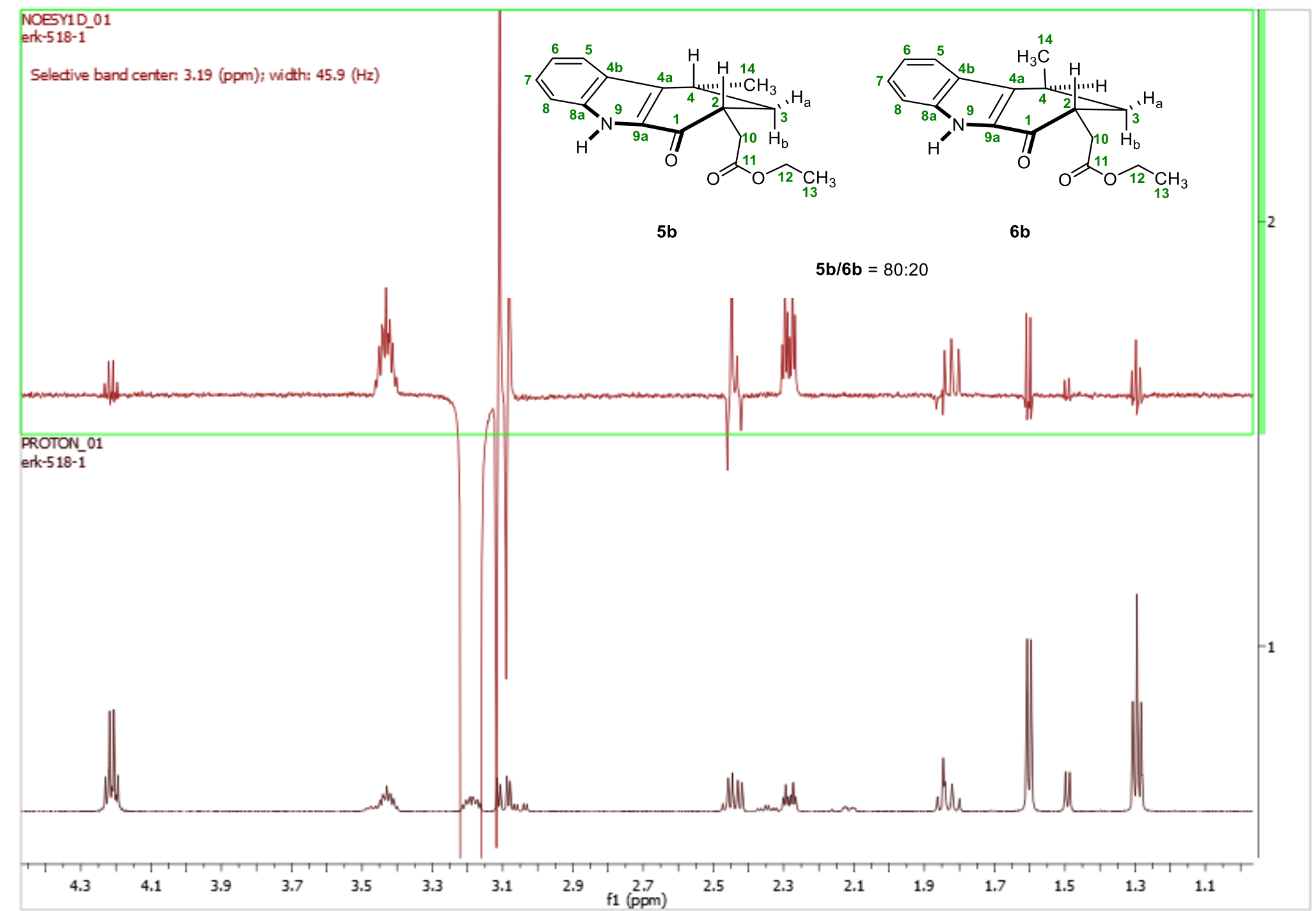

NOE-DIFF Spectrum of $5 \mathrm{~b} / \mathbf{6 b}\left(\mathbf{6 0 0} \mathrm{MHz}, \mathrm{CDCl}_{3}\right)$ : Enhancement of $\boldsymbol{H}-4, \boldsymbol{H}_{\mathbf{a}}-\mathbf{3}$ and $\mathbf{H 1 0}$ signals of $\mathbf{5 b}$ after irradiation of $\boldsymbol{H}-\mathbf{2}$ signal at $3.19 \mathrm{ppm}$. 


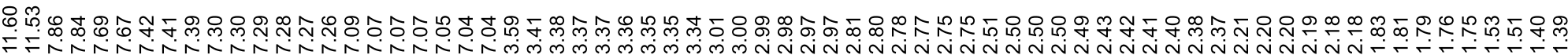

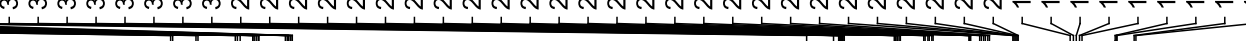
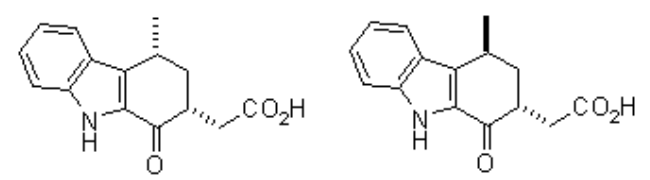

$7 \mathbf{b} \quad 7 \mathbf{b} / 8 \mathbf{b}=80: 20$

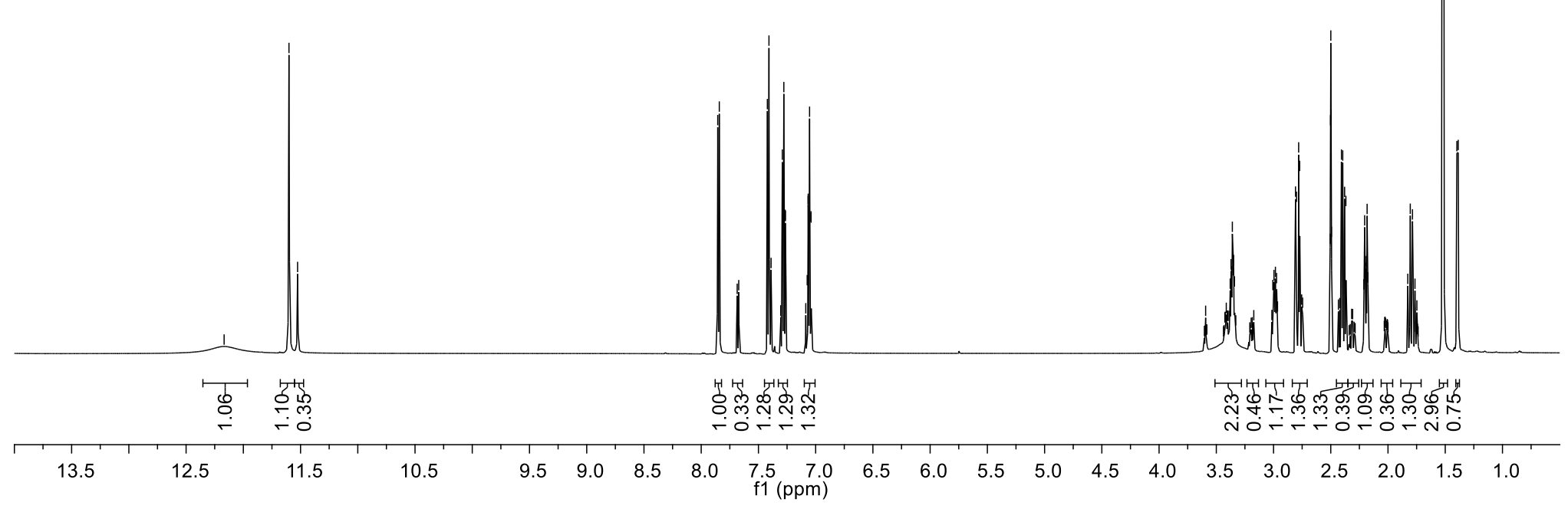

${ }^{1} \mathrm{H}-\mathrm{NMR}$ Spectrum of $7 \mathrm{~b} / 8 \mathrm{~b}\left(600 \mathrm{MHz}\right.$, DMSO- $\left.d_{6}\right)$ 


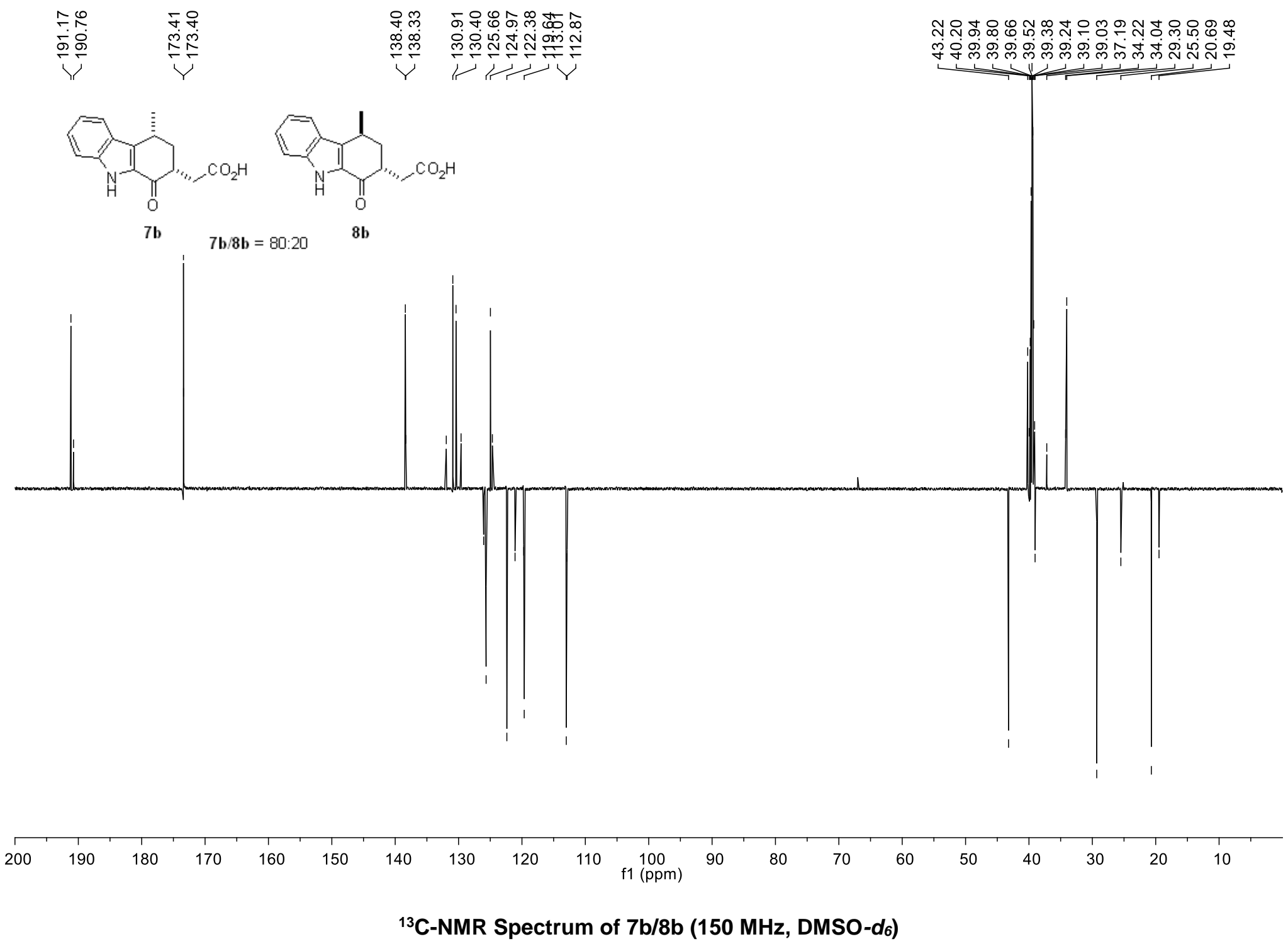


Table S3. NMR spectral data of compound $9 \mathbf{b}$.

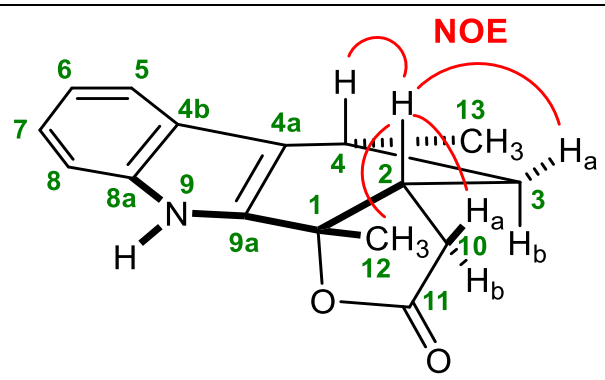

$9 b$

\begin{tabular}{|c|c|c|c|c|c|}
\hline $\mathrm{C} \#$ & $\delta_{\mathrm{C}}$ & $\delta_{\mathrm{H}}$ & Mult. & COSY & NOE-DIFF \\
\hline 1 & 80.5 & - & $\mathrm{C}$ & & \\
\hline 2 & 40.0 & $2.56-2.50(\mathrm{~m}, 1 \mathrm{H}, H-2)$ & $\mathrm{CH}$ & $2 \times H-10, H_{a}-3, H_{b}-3$ & $\begin{array}{l}H_{a}-10, H-4, \\
H-12, H_{a}-3\end{array}$ \\
\hline 3 & 37.5 & $1.92\left(\operatorname{td}, J=13.3,4.4 \mathrm{~Hz}, H_{a}-3\right)$ & $\mathrm{CH}_{2}$ & $H_{a}-3$ or $H_{b}-3, H-2, H-4$ & \\
\hline 3 & & $1.22-1.16\left(\mathrm{~m}, 1 \mathrm{H}, H_{b^{-}}-3\right)$ & & $H_{a}-3$ or $H_{b}-3, H-2, H-4$ & \\
\hline 4 & 27.4 & $2.99-2.95(\mathrm{~m}, 1 \mathrm{H}, H-4)$ & $\mathrm{CH}$ & $H-13, H_{a}-3, H_{b}-3$ & \\
\hline $4 \mathbf{a}$ & 116.3 & - & $\mathrm{C}$ & & \\
\hline $4 b$ & 125.2 & - & $\mathrm{C}$ & & \\
\hline 5 & 118.6 & $6.97(\mathrm{t}, J=7.3 \mathrm{~Hz}, 1 \mathrm{H}, H-5)$ & $\mathrm{CH}$ & & \\
\hline 6 & 121.8 & $7.11(\mathrm{t}, J=7.3 \mathrm{~Hz}, 1 \mathrm{H}, H-6)$ & $\mathrm{CH}$ & & \\
\hline 7 & 120.6 & $7.67(\mathrm{~d}, J=8.0 \mathrm{~Hz}, 1 \mathrm{H}, H-7)$ & $\mathrm{CH}$ & & \\
\hline 8 & 111.6 & $7.36(\mathrm{t}, J=8.1 \mathrm{~Hz}, 1 \mathrm{H}, H-8)$ & $\mathrm{CH}$ & & \\
\hline $8 \mathbf{a}$ & 136.9 & $C-8 \mathrm{a}$ or $C-9 \mathrm{a}$ & $\mathrm{C}$ & & \\
\hline & - & $11.28(\mathrm{~s}, \mathrm{~N}-H)$ & - & & \\
\hline $9 \mathrm{a}$ & 132.2 & $C-8 \mathrm{a}$ or $C-9 \mathrm{a}$ & $\mathrm{C}$ & & \\
\hline 10 & 35.4 & $3.23\left(\mathrm{dd}, J=17.6,7.8 \mathrm{~Hz}, 1 \mathrm{H}, H_{a^{-}}-10\right)$ & $\mathrm{CH}_{2}$ & $H-10, H-2$ & \\
\hline 10 & & $2.25\left(\mathrm{~d}, J=17.5 \mathrm{~Hz}, 1 \mathrm{H}, H_{b}-10\right)$ & & $H-10, H-2$ & \\
\hline 11 & 175.8 & $\mathrm{C}=\mathrm{O}$ & $\mathrm{C}$ & & \\
\hline 12 & 25.1 & $1.69(\mathrm{~s}, 3 \mathrm{H}, H-12)$ & $\mathrm{CH}_{3}$ & & \\
\hline 13 & 20.7 & $1.43(\mathrm{~d}, J=6.8 \mathrm{~Hz}, 3 \mathrm{H}, H-13)$ & $\mathrm{CH}_{3}$ & $H-4$ & \\
\hline
\end{tabular}




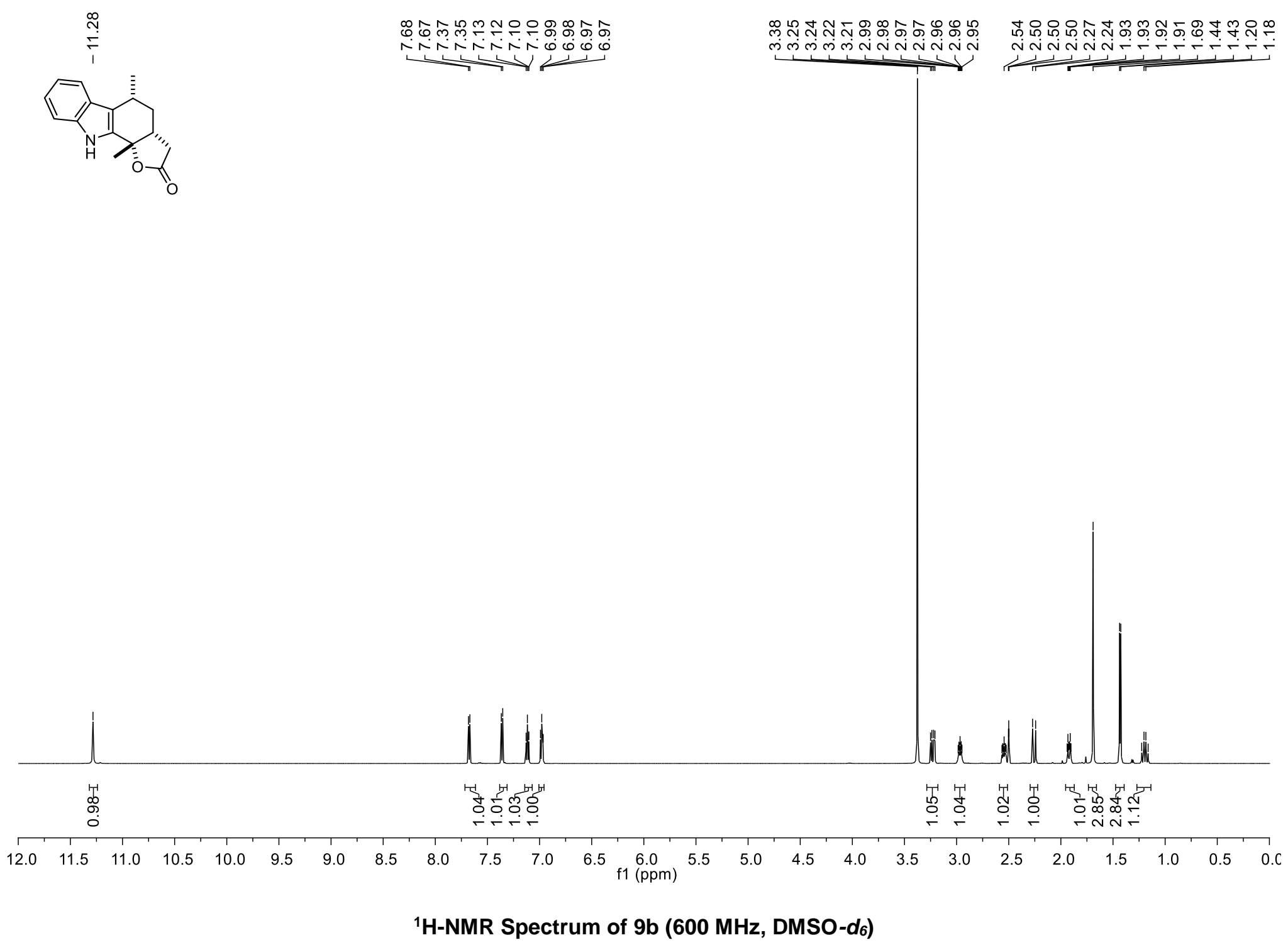




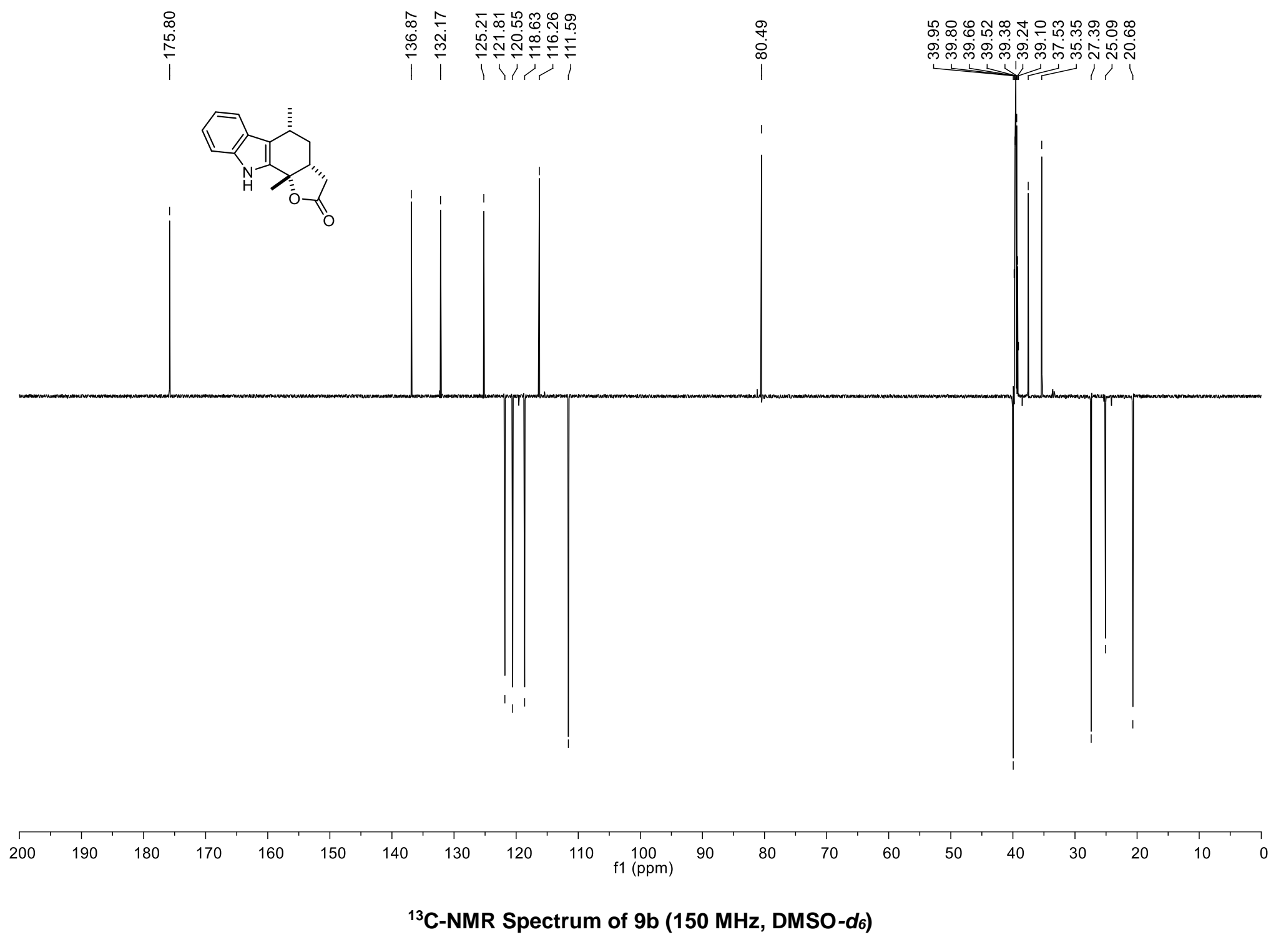




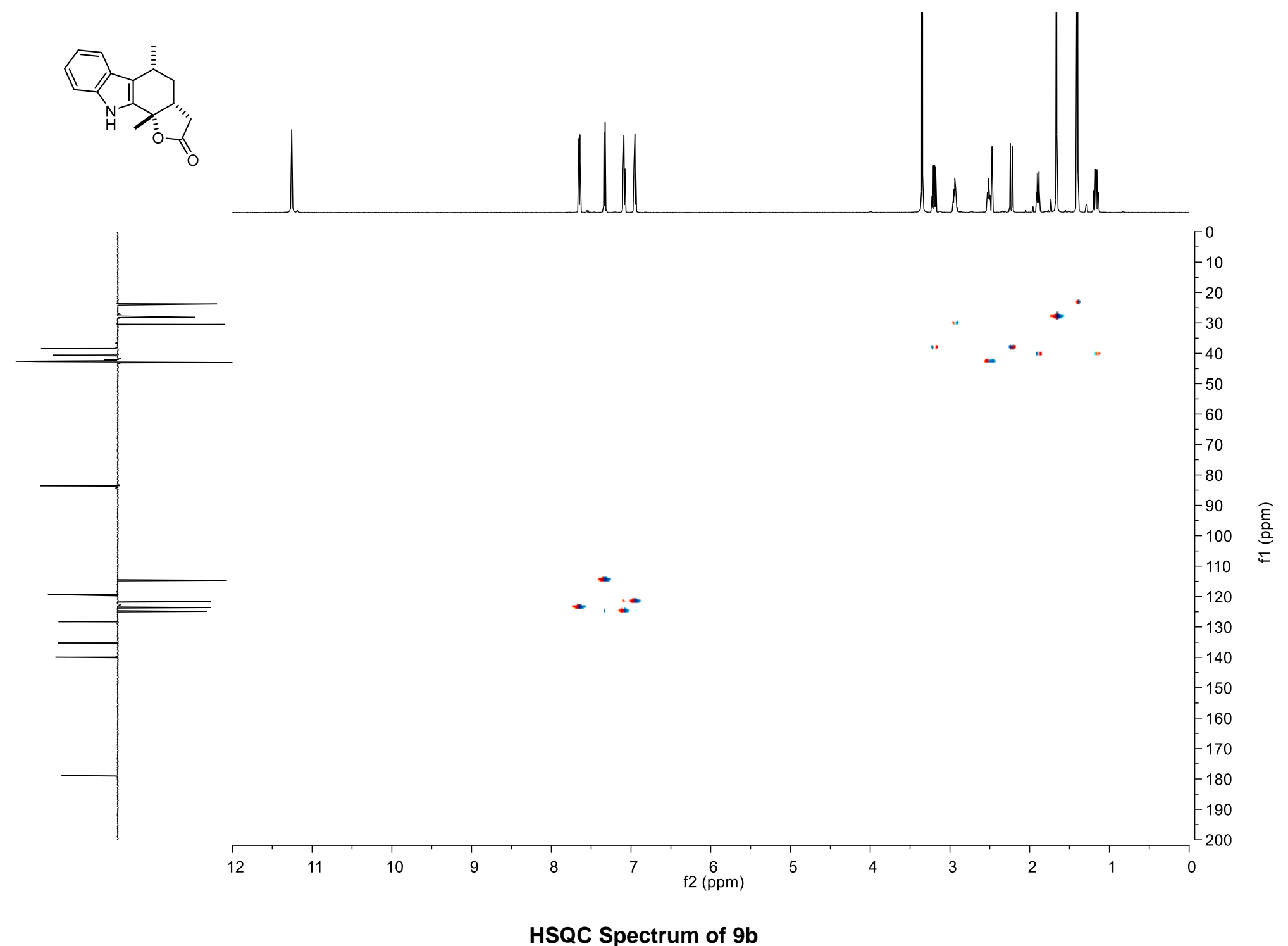




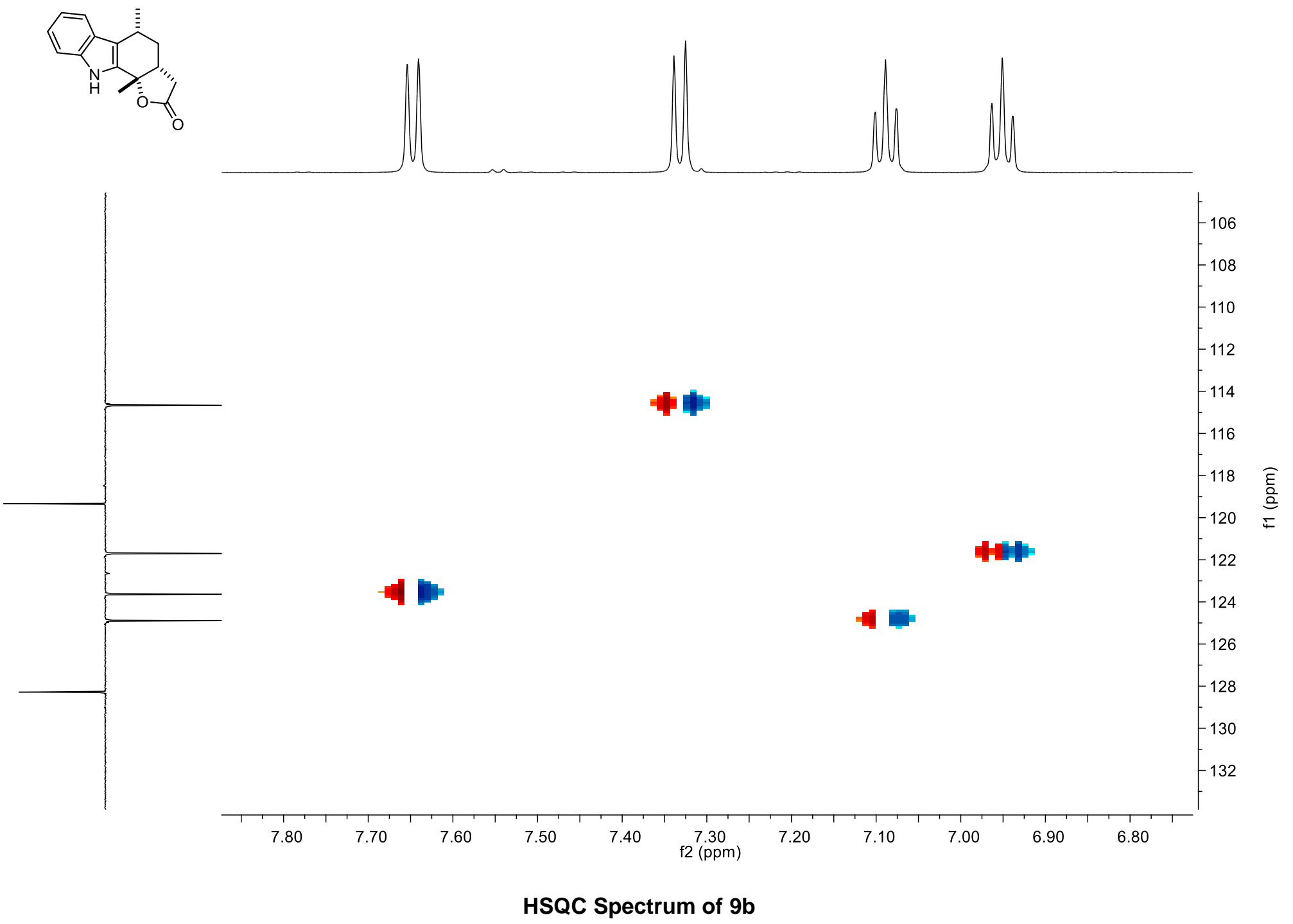



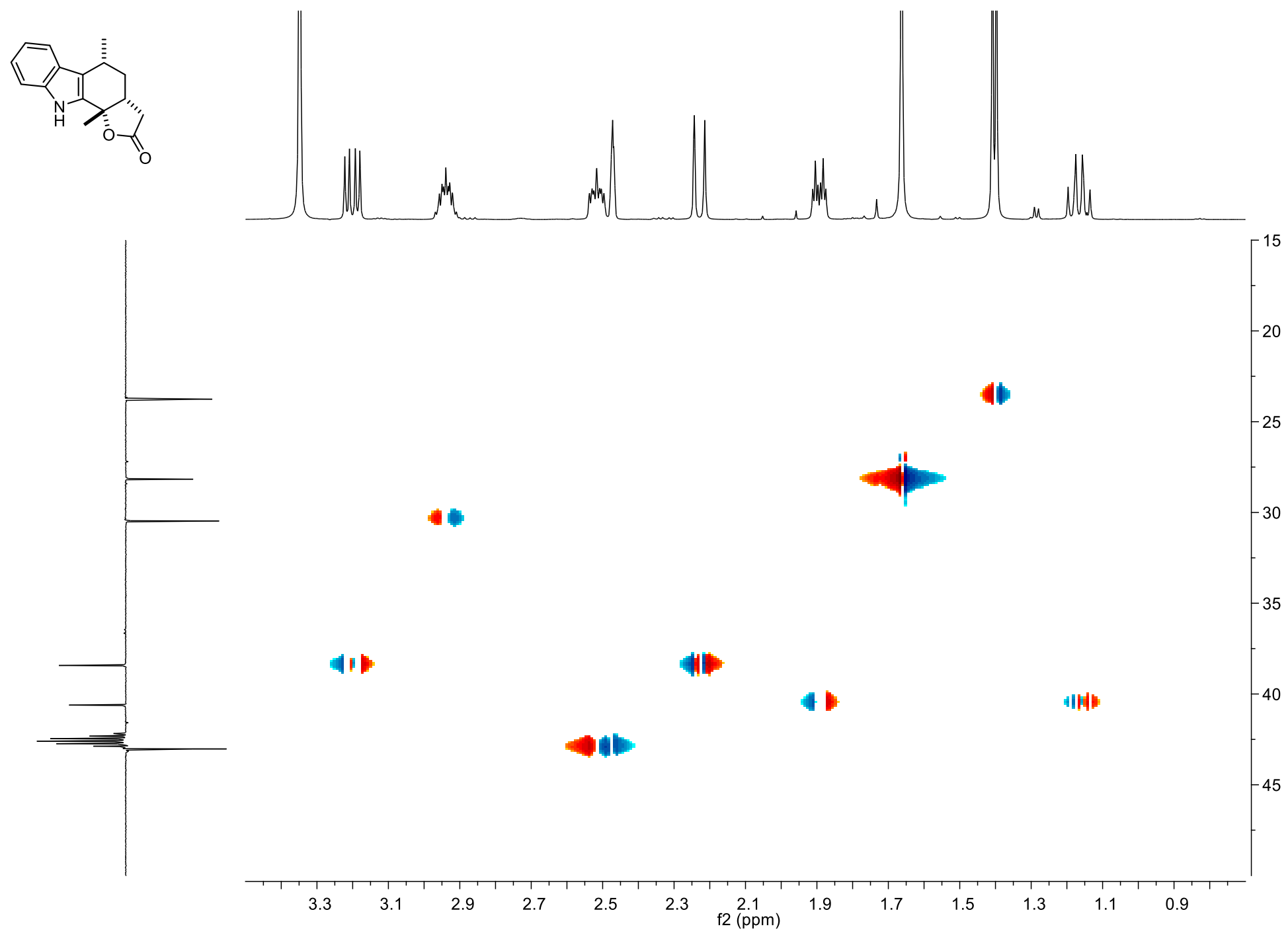

HSQC Spectrum of 9b 


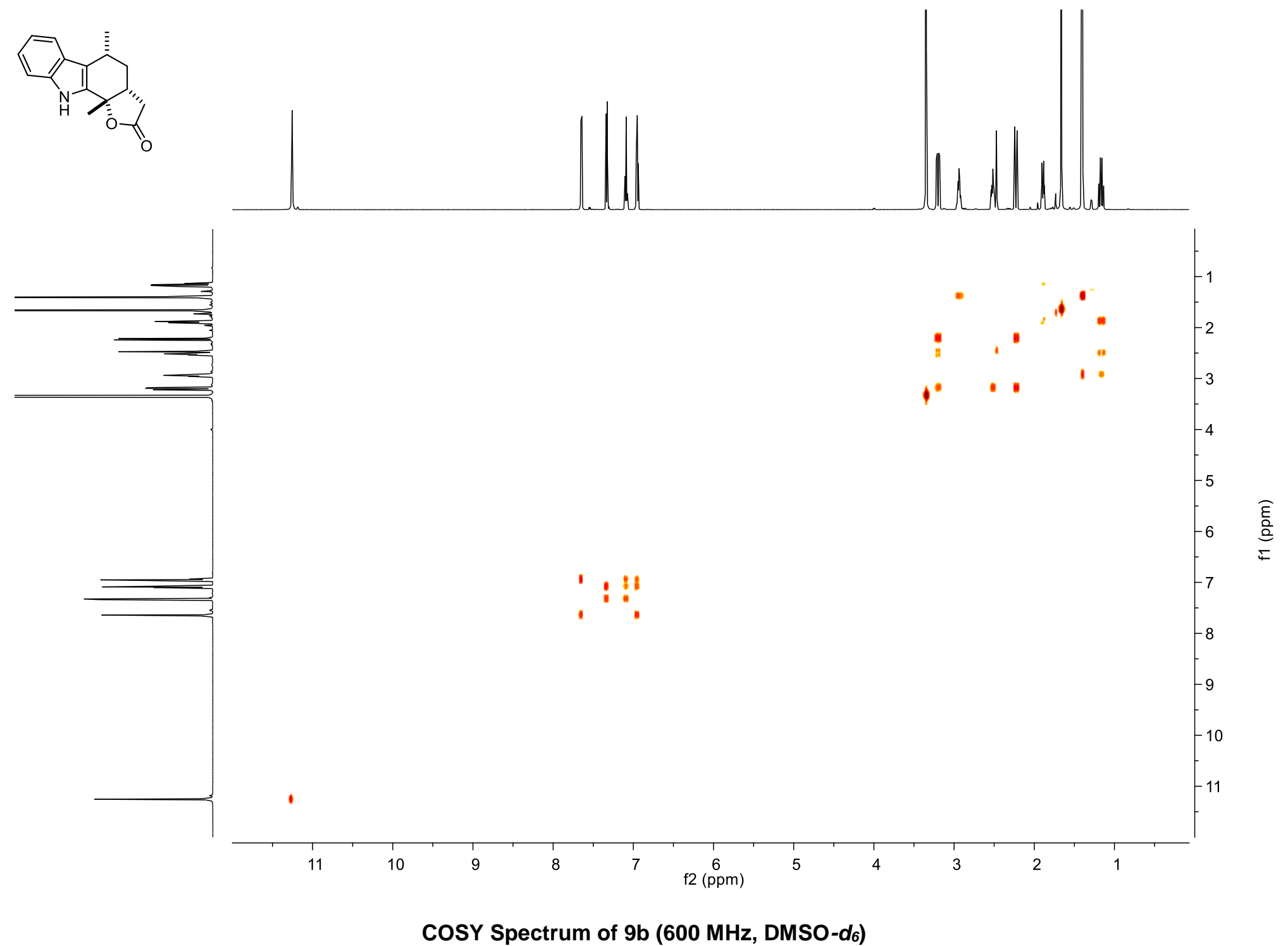




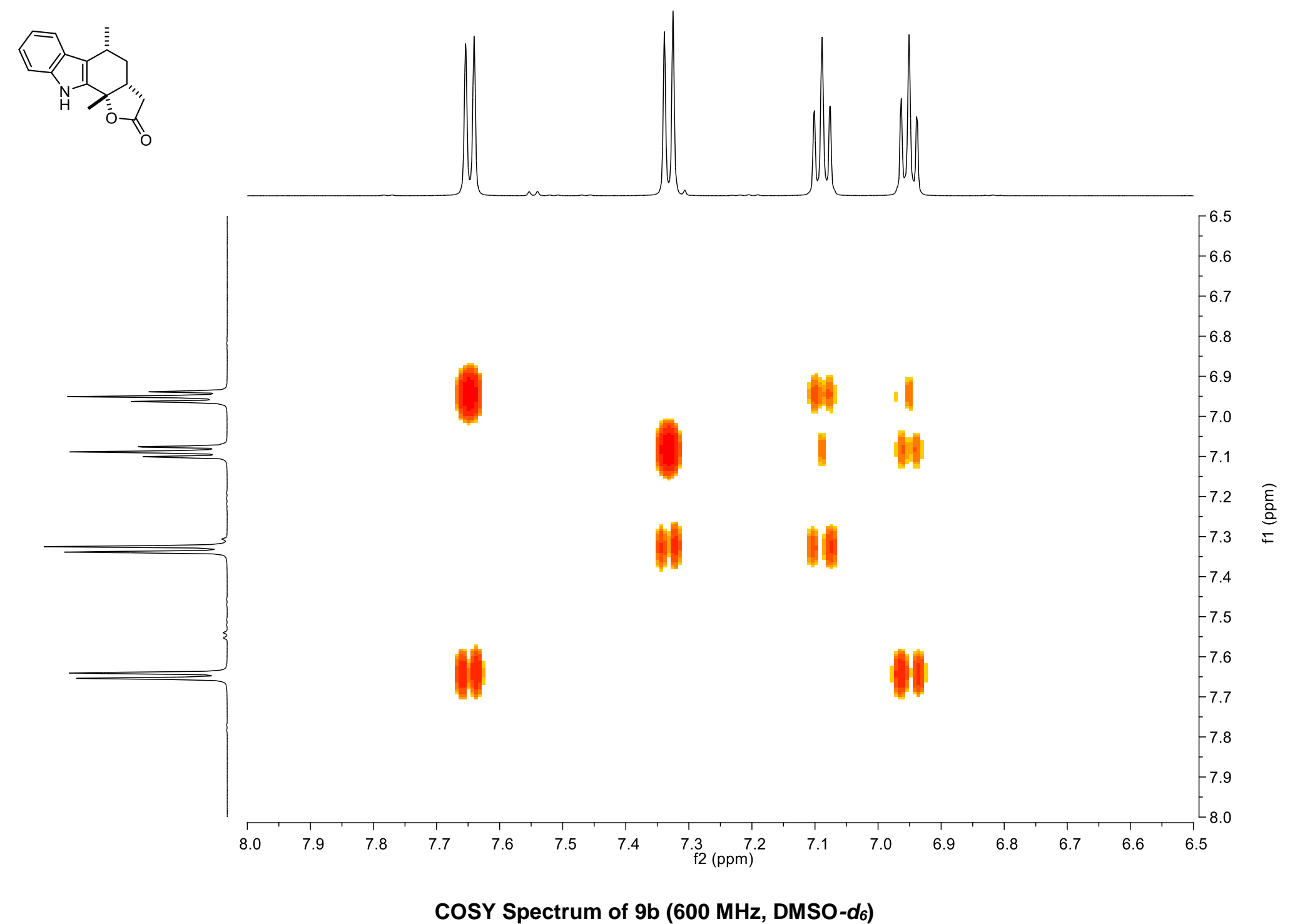




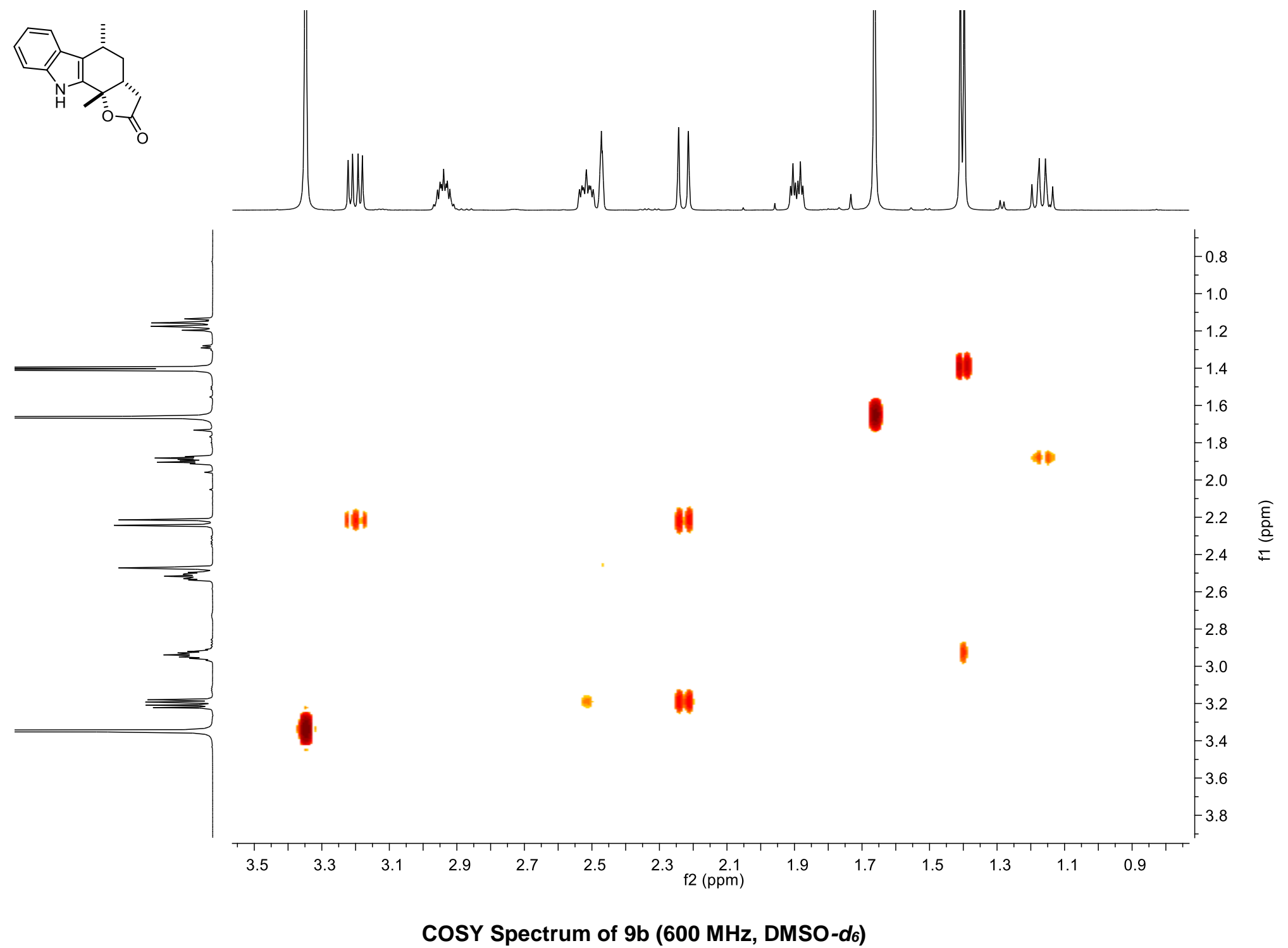




\section{NOESY1D_01}

Selective band center: 2.52 (ppm); width: $41.2(\mathrm{~Hz})$

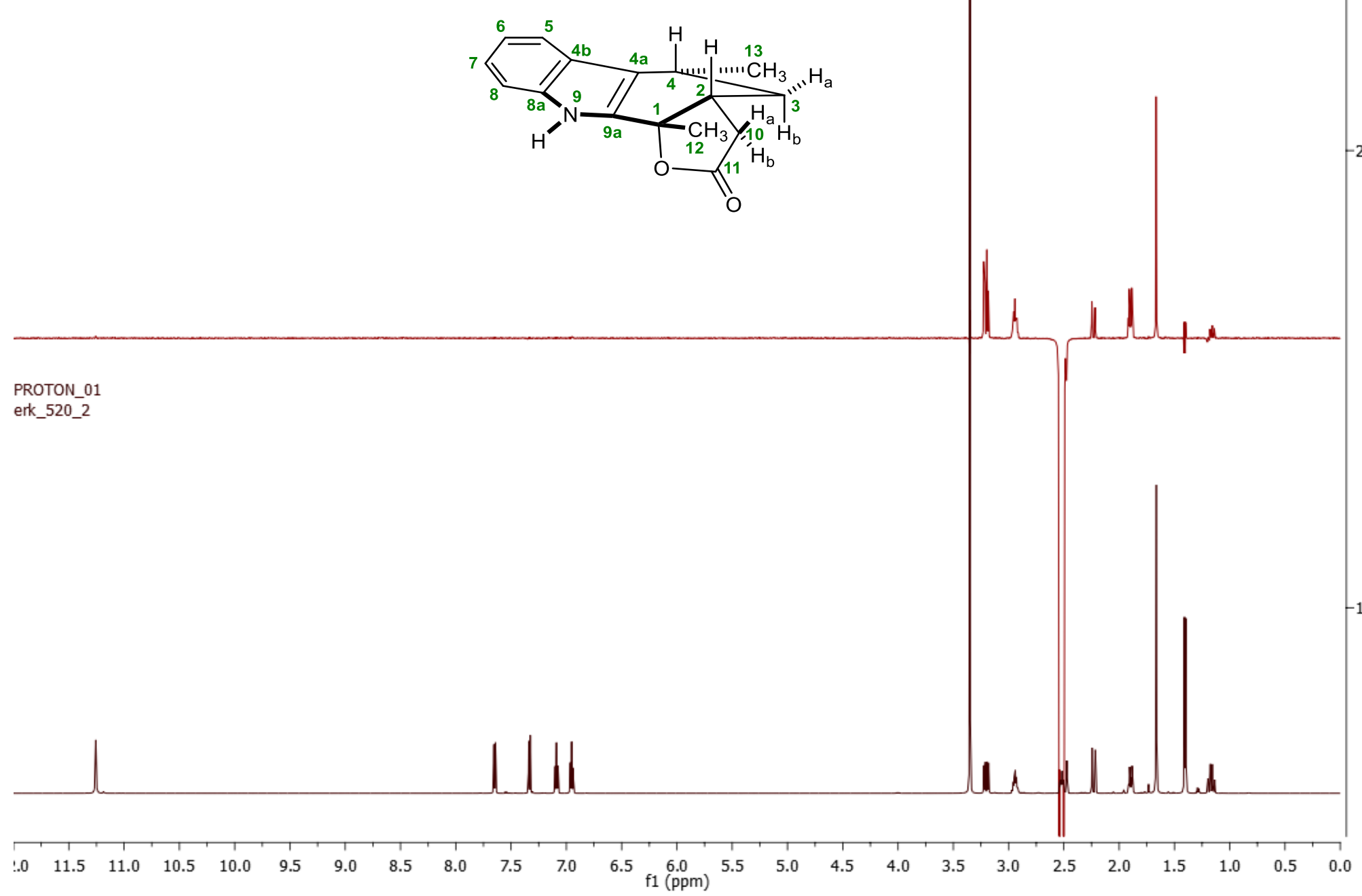

NOE-DIFF Spectrum of 9b (600 MHz, DMSO-d $)$ : Enhancement of $\boldsymbol{H - 4}, \boldsymbol{H}-\mathbf{1 2}, \boldsymbol{H}_{\mathbf{a}}-\mathbf{3}$ and $\boldsymbol{H}_{\mathbf{a}}-\mathbf{1 0}$ signals after irradiation of $\boldsymbol{H}-\mathbf{2}$ signal at 2.52 ppm 


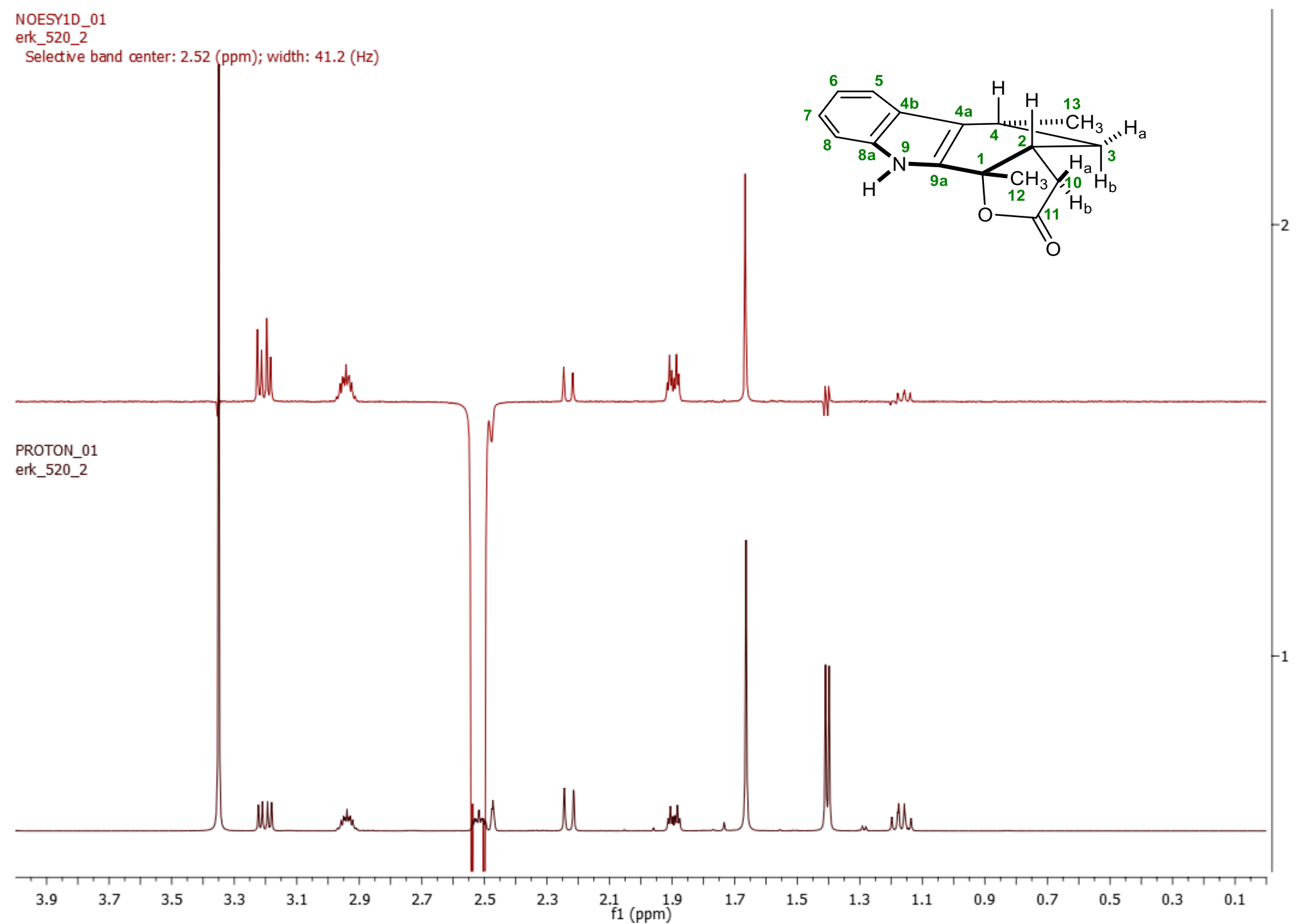

NOE-DIFF Spectrum of $9 \mathrm{~b}\left(600 \mathrm{MHz}\right.$, DMSO-d $\boldsymbol{d}_{6}$ : Enhancement of $\boldsymbol{H - 4}, \boldsymbol{H}-\mathbf{1 2}, \boldsymbol{H}_{\mathbf{a}}-\mathbf{3}$ and $\boldsymbol{H}_{\mathbf{a}}-\mathbf{1 0}$ signals after irradiation of $\boldsymbol{H}-\mathbf{2}$ signal at 2.52 ppm 
Table S4. NMR spectral data of compound 10b.

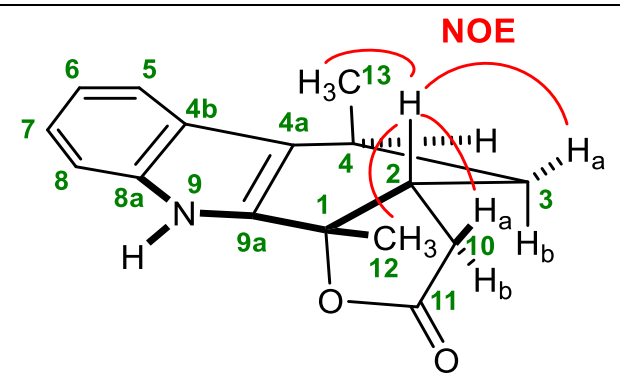

$10 b$

\begin{tabular}{|c|c|c|c|c|c|}
\hline $\mathrm{C} \#$ & $\delta_{\mathrm{C}}$ & $\delta_{\mathrm{H}}$ & Mult. & COSY & NOE-DIFF \\
\hline $\mathbf{1}$ & 81.2 & - & $\mathrm{C}$ & & \\
\hline 2 & 38.5 & $2.75(\mathrm{~m}, 1 \mathrm{H}, H-2)$ & $\mathrm{CH}$ & $\begin{array}{l}H_{a}-3, H_{b}-3, \\
2 \times H-10\end{array}$ & $\begin{array}{l}H-13, H-12, \\
H_{a}-3, H_{a}-10\end{array}$ \\
\hline 3 & 33.4 & $1.85-1.81\left(\mathrm{~m}, 1 \mathrm{H}, H_{b^{-}}-3\right)$ & $\mathrm{CH}_{2}$ & $H-4, H_{a}-3, H-2$ & \\
\hline 3 & - & $1.71-1.67\left(\mathrm{~m}, 1 \mathrm{H}, H_{a^{-}}-3\right)$ & & $H-4, H_{b}-3, H-2$ & \\
\hline 4 & 24.1 & $3.17-3.14(\mathrm{~m}, 1 \mathrm{H}, H-4)$ & $\mathrm{CH}$ & $H_{a}-3, H_{b}-3, H-13$ & \\
\hline $4 a$ & 115.4 & - & $\mathrm{C}$ & & \\
\hline 4b & 125.2 & - & $\mathrm{C}$ & & \\
\hline 5 & 118.6 & $6.99(\mathrm{t}, J=7.5 \mathrm{~Hz}, 1 \mathrm{H}, H-5)$ & $\mathrm{CH}$ & & \\
\hline 6 & 121.9 & $7.11(\mathrm{t}, J=7.4 \mathrm{~Hz}, 1 \mathrm{H}, H-6)$ & $\mathrm{CH}$ & & \\
\hline 7 & 119.6 & $7.57(\mathrm{~d}, J=7.9 \mathrm{~Hz}, 1 \mathrm{H}, H-7)$ & $\mathrm{CH}$ & & \\
\hline 8 & 111.5 & $7.34(\mathrm{~d}, J=8.1 \mathrm{~Hz}, 1 \mathrm{H}, H-8)$ & $\mathrm{CH}$ & & \\
\hline 8a & 136.8 & $C-8 \mathrm{a}$ or $C-9 \mathrm{a}$ & $\mathrm{C}$ & & \\
\hline 9 & & $11.22(\mathrm{~s}, \mathrm{~N}-H)$ & - & & \\
\hline 9a & 132.3 & $C-8 \mathrm{a}$ or $C-9 \mathrm{a}$ & $\mathrm{C}$ & & \\
\hline 10 & 33.6 & $2.36\left(\mathrm{dd}, J=17.6,6.4 \mathrm{~Hz}, 1 \mathrm{H}, H_{b^{-}} 10\right)$ & $\mathrm{CH}_{2}$ & $H-10, H-2$ & \\
\hline 10 & - & $2.91\left(\mathrm{dd}, J=17.6,8.3 \mathrm{~Hz}, 1 \mathrm{H}, H_{a^{-}} 10\right)$ & & $H-10, H-2$ & \\
\hline 11 & 175.8 & $\mathrm{C}=\mathrm{O}$ & $\mathrm{C}$ & & \\
\hline 12 & 25.3 & $1.76(\mathrm{~s}, 3 \mathrm{H}, H-12)$ & $\mathrm{CH}_{3}$ & & \\
\hline 13 & 20.6 & $1.31(\mathrm{~d}, J=6.9 \mathrm{~Hz}, 3 \mathrm{H}, H-13)$ & $\mathrm{CH}_{3}$ & $H-4$ & \\
\hline
\end{tabular}




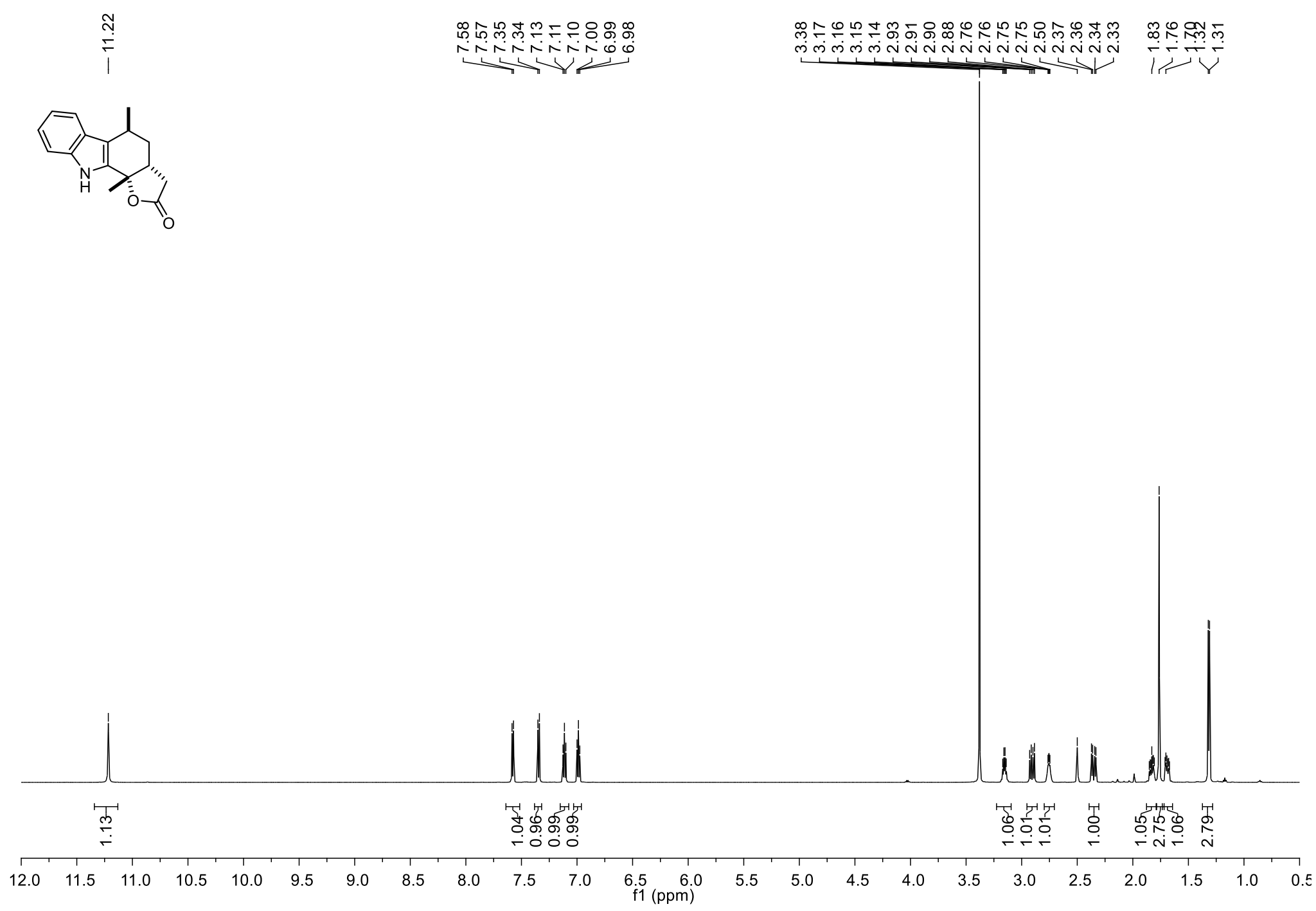

${ }^{1} \mathrm{H}-\mathrm{NMR}$ Spectrum of $10 \mathrm{~b}\left(600 \mathrm{MHz}\right.$, DMSO- $\left.d_{6}\right)$ 


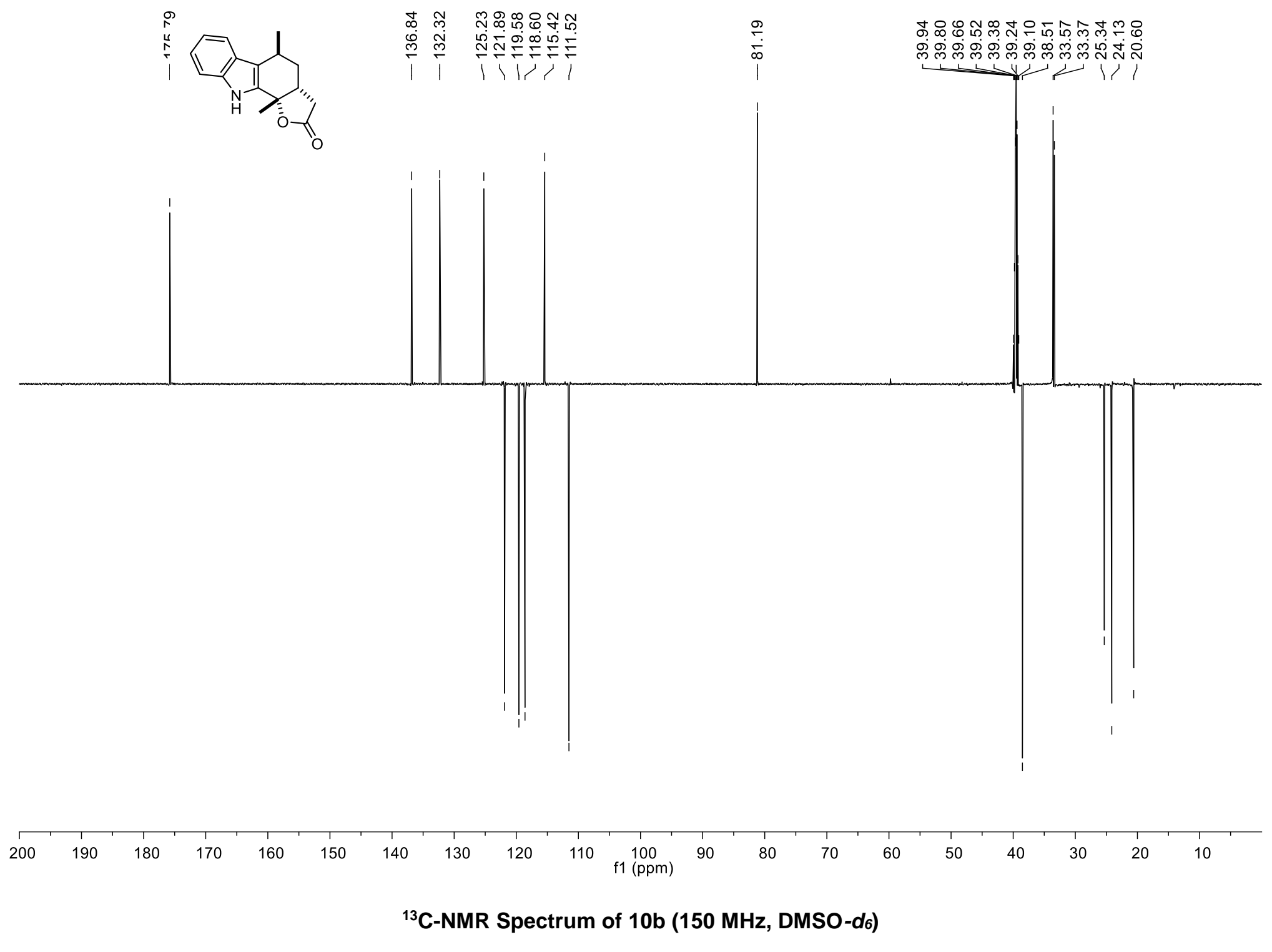



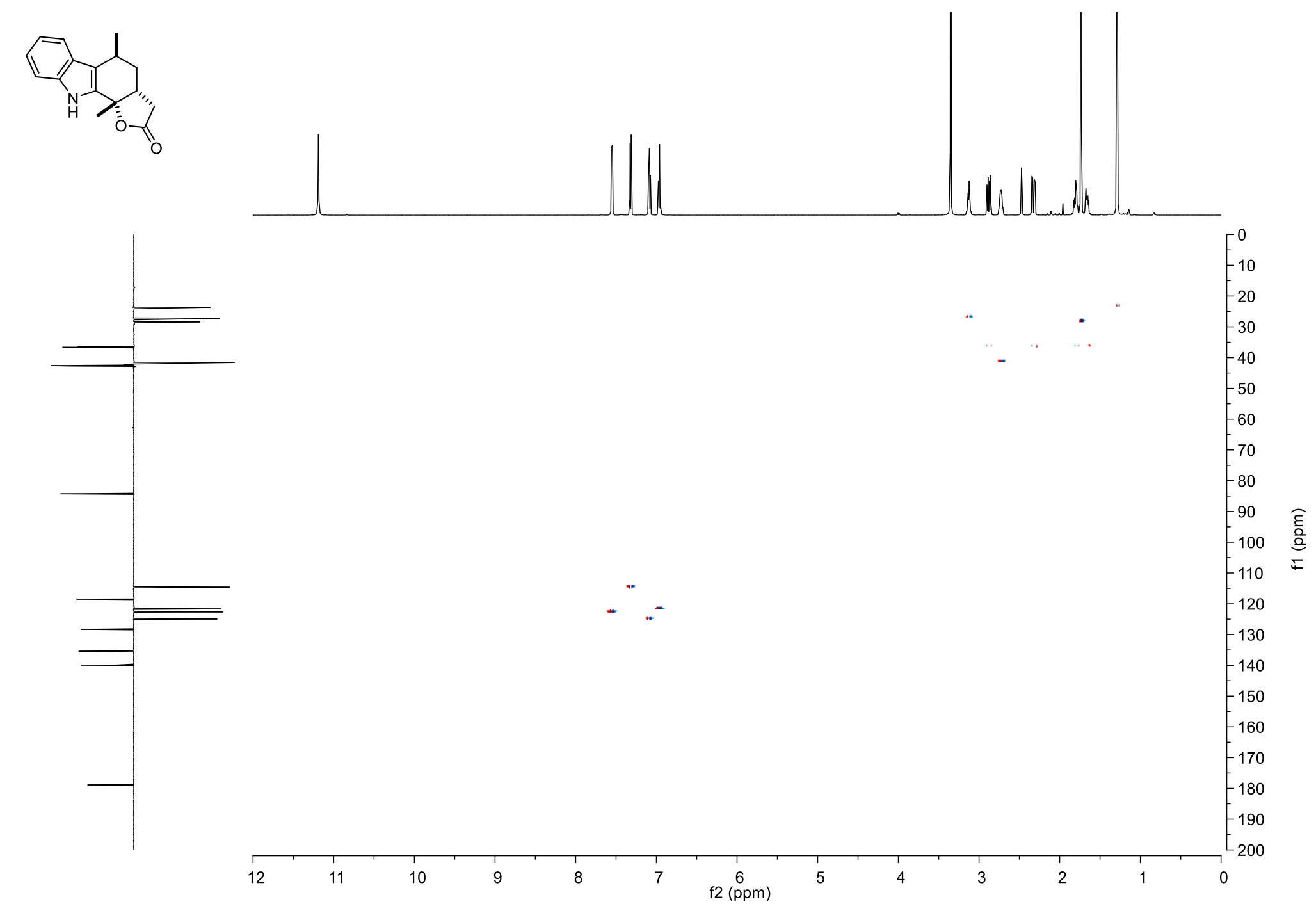

HSQC Spectrum of 10b 


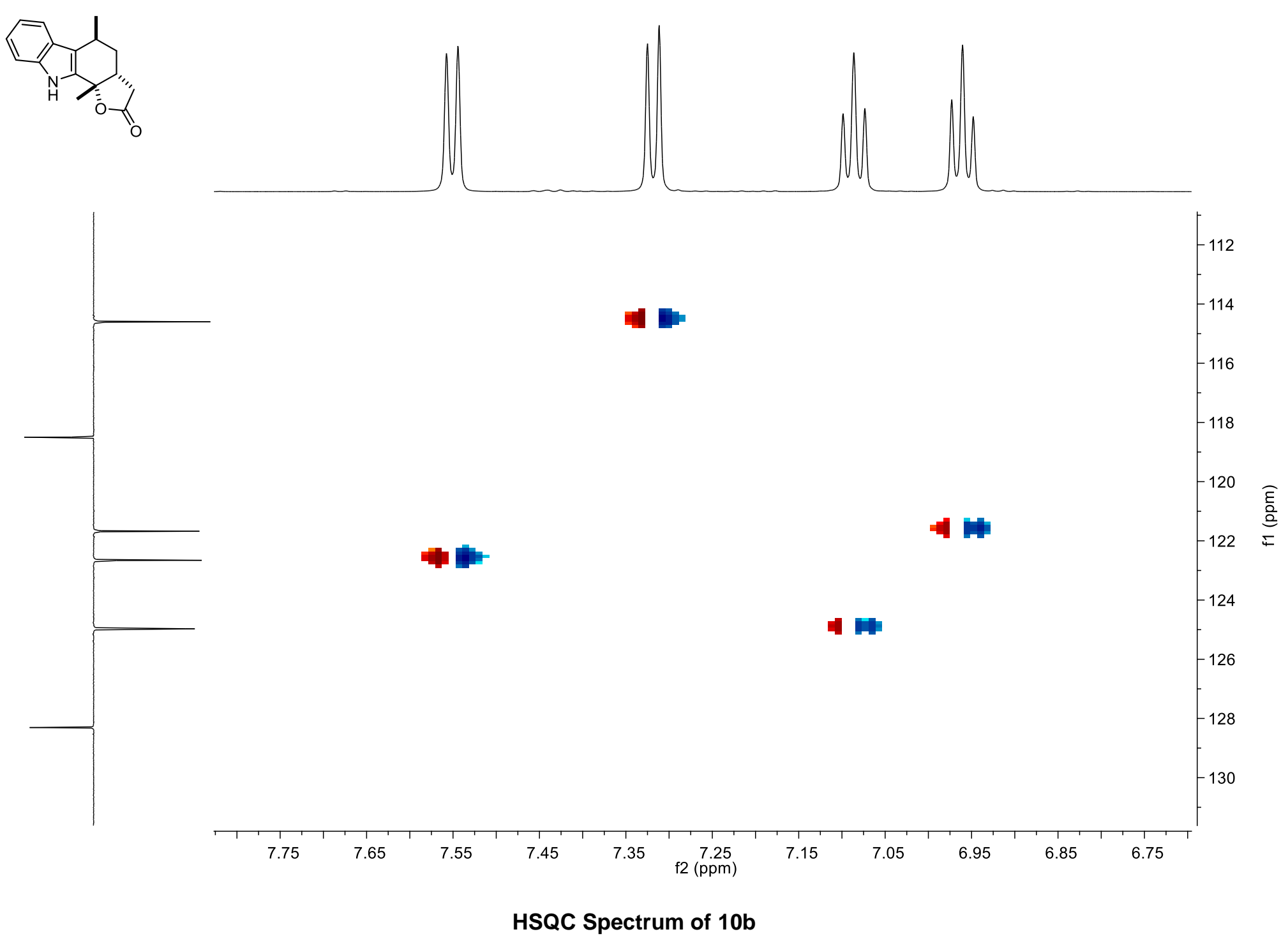




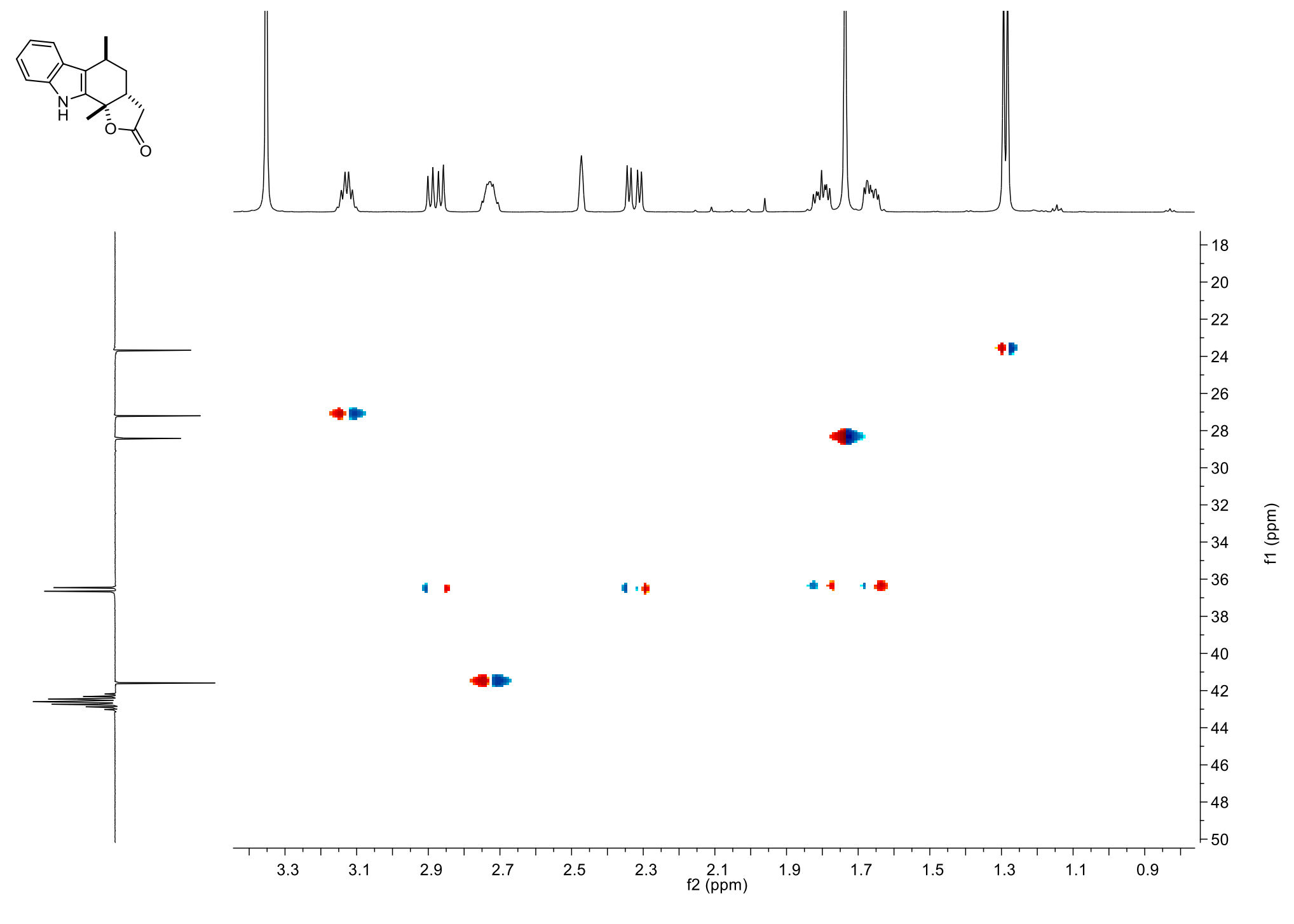

HSQC Spectrum of 10b 


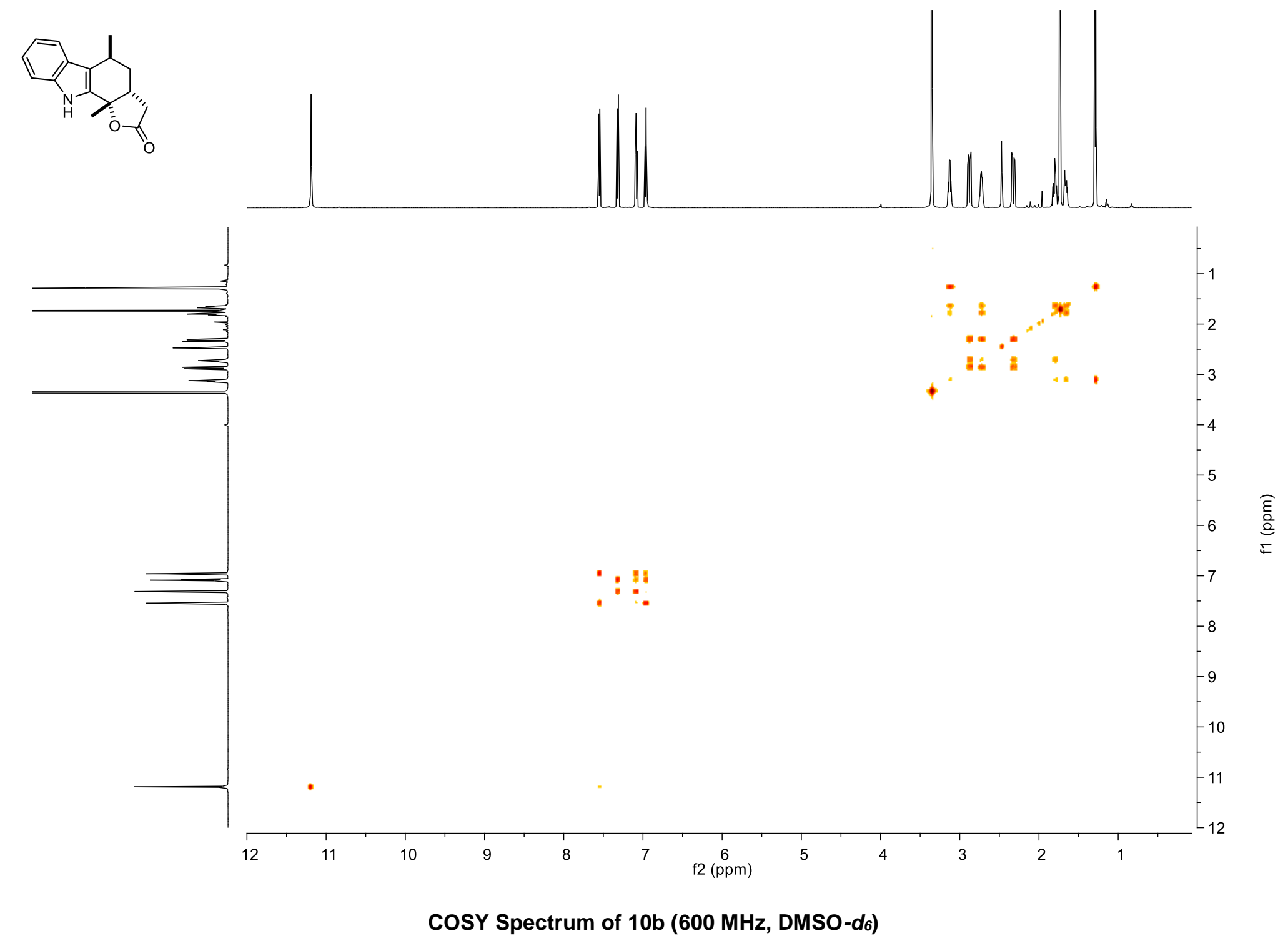




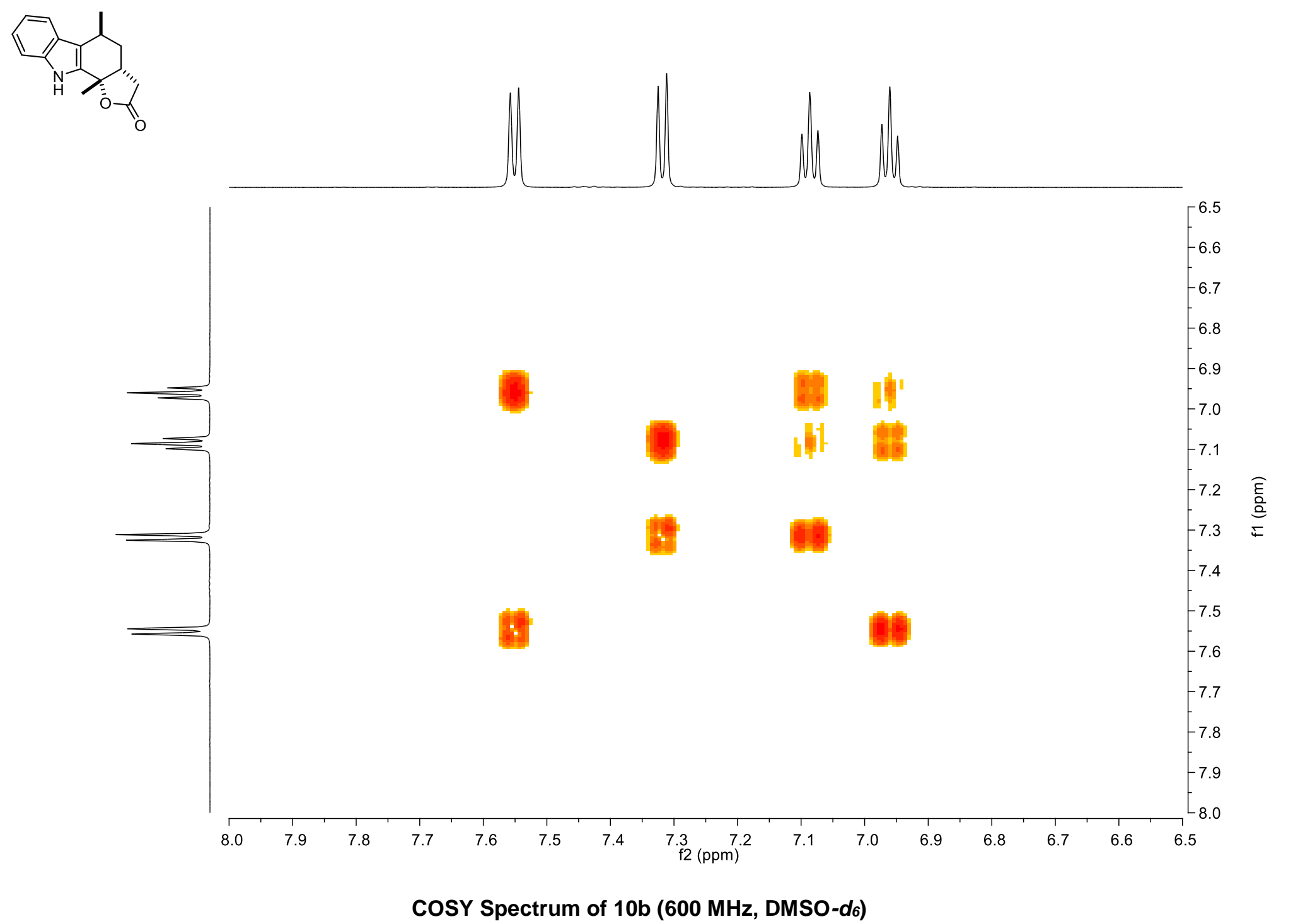




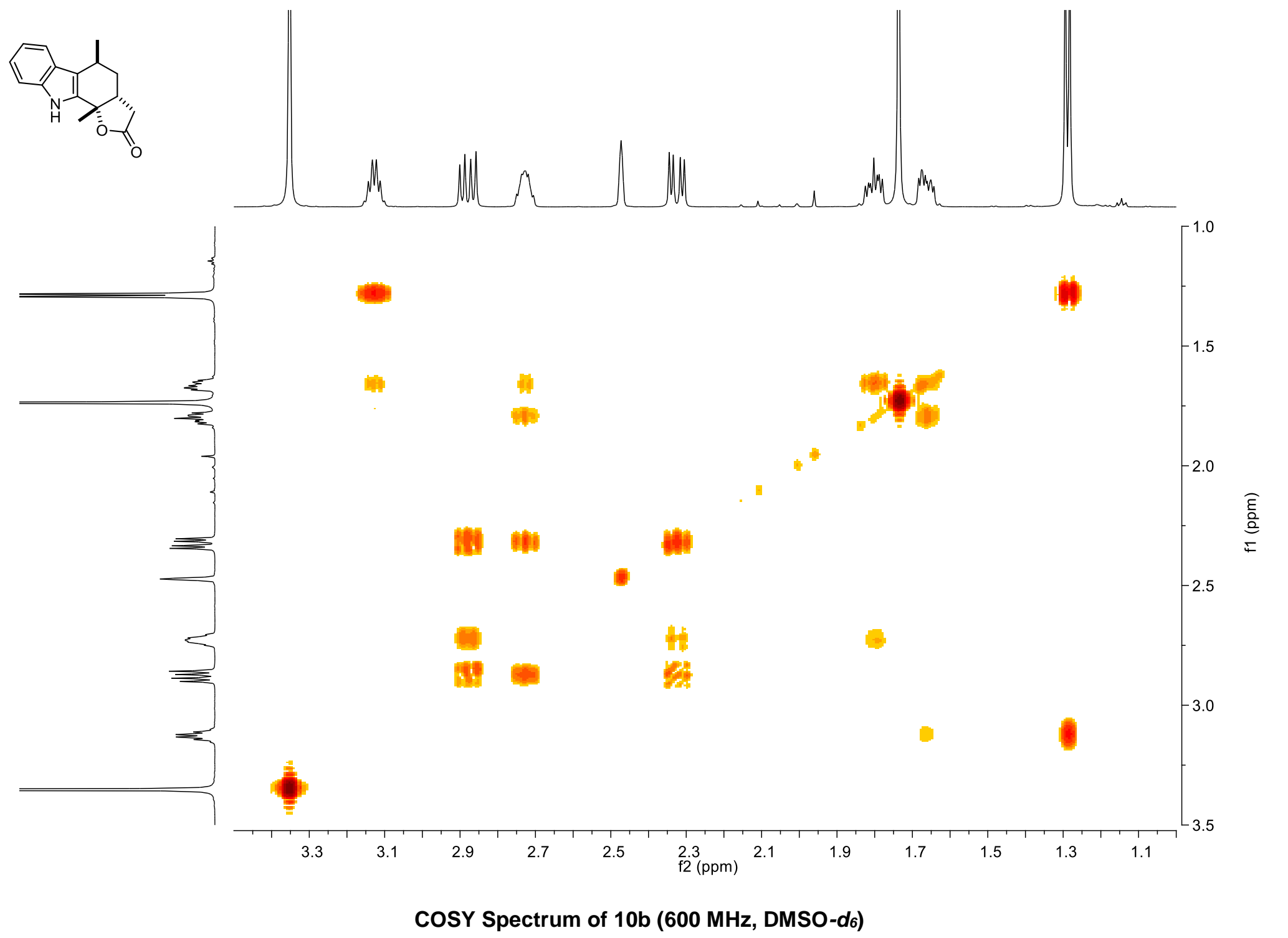


Selective band œnter: $2.73(\mathrm{ppm})$; width: $59.6(\mathrm{~Hz})$

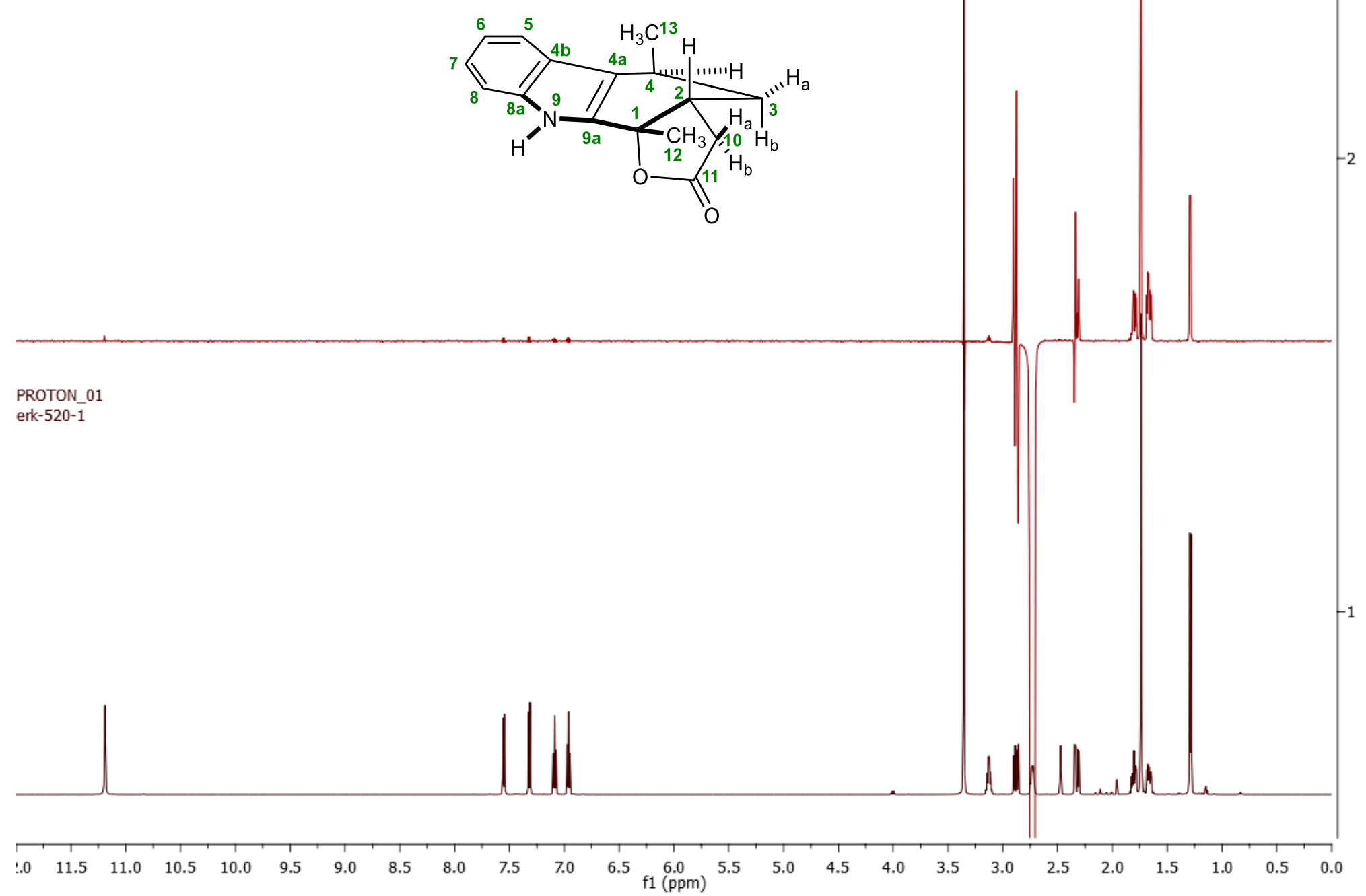

NOE-DIFF Spectrum of $10 \mathrm{~b}\left(600 \mathrm{MHz}\right.$, DMSO- $\left.\boldsymbol{d}_{6}\right)$ : Enhancement of $\boldsymbol{H - 1 3}, \boldsymbol{H - 1 2}, \boldsymbol{H}_{\mathbf{a}}-3$ and $\boldsymbol{H}_{\mathbf{a}}-\mathbf{1 0}$ signals after irradiation of $\boldsymbol{H}-2$ signal at 2.73 ppm 


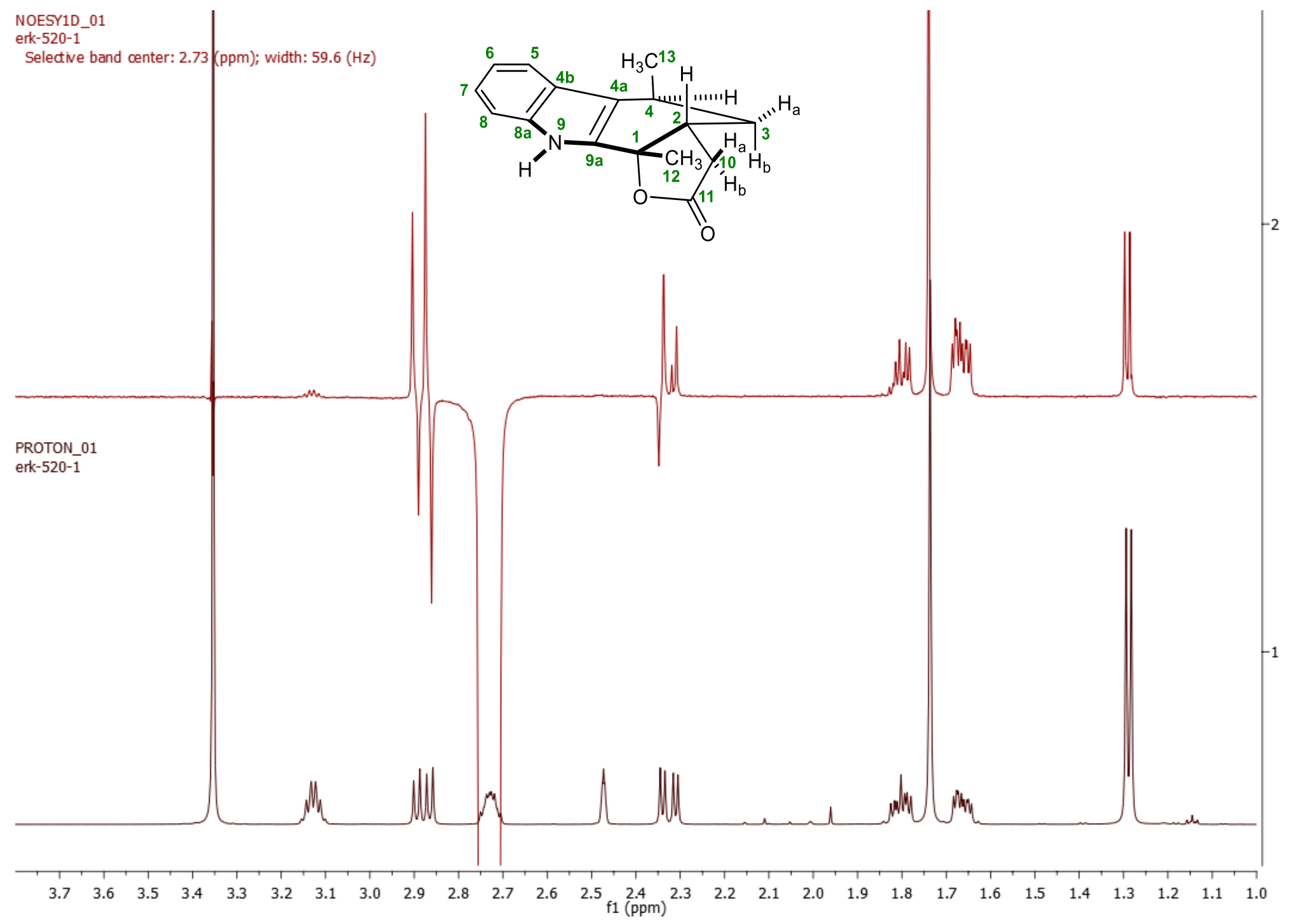

NOE-DIFF Spectrum of $10 \mathrm{~b}\left(600 \mathrm{MHz}\right.$, DMSO- $\left.\boldsymbol{d}_{6}\right)$ : Enhancement of $\boldsymbol{H - 1 3}, \boldsymbol{H}-12, \boldsymbol{H}_{\mathbf{a}}-\mathbf{3}$ and $\boldsymbol{H}_{\mathbf{a}}-\mathbf{1 0}$ signals after irradiation of $\boldsymbol{H - 2}$ signal at 2.73 ppm 


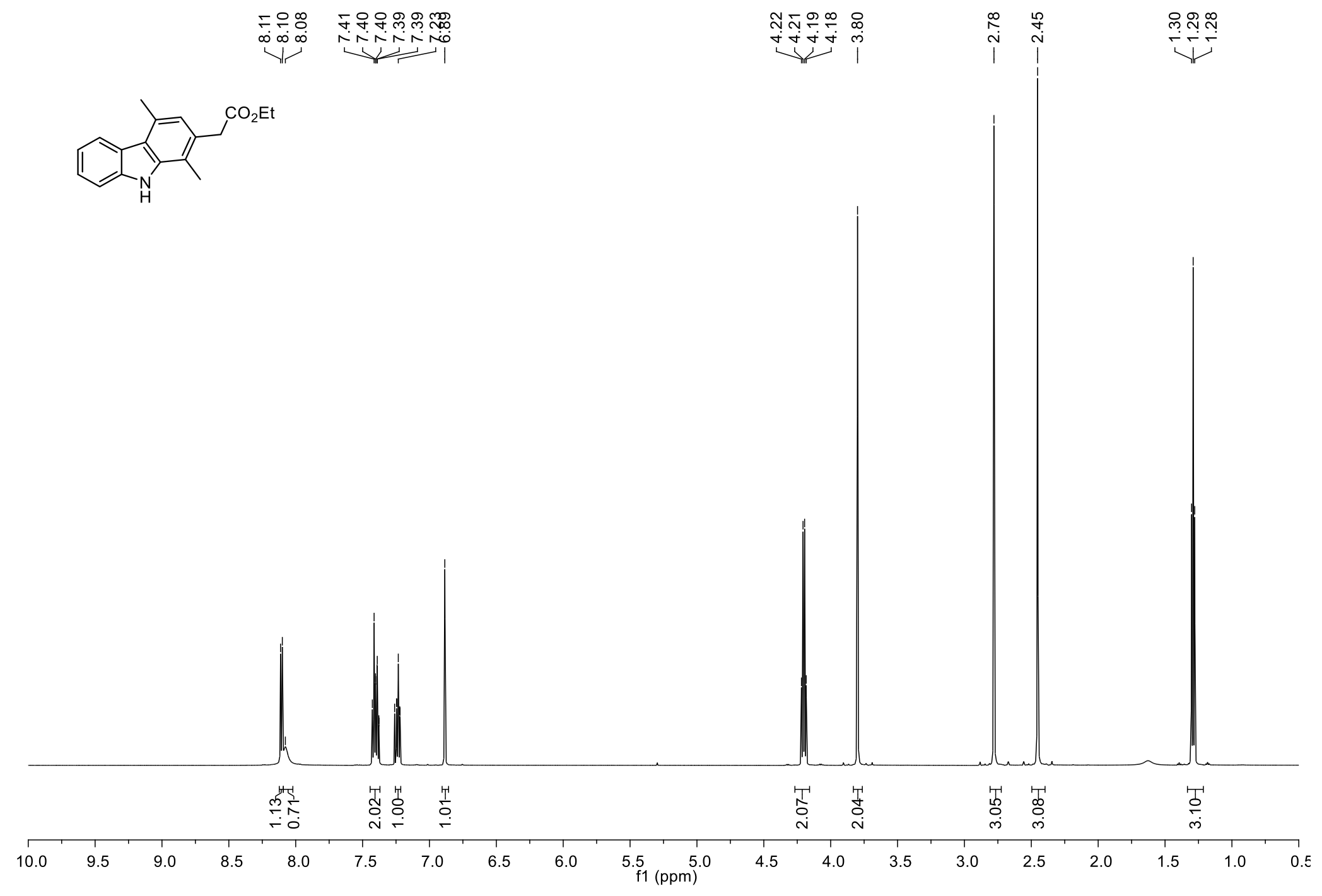

${ }^{1} \mathrm{H}-\mathrm{NMR}$ Spectrum of $15 \mathrm{~b}\left(600 \mathrm{MHz}, \mathrm{CDCl}_{3}\right)$ 


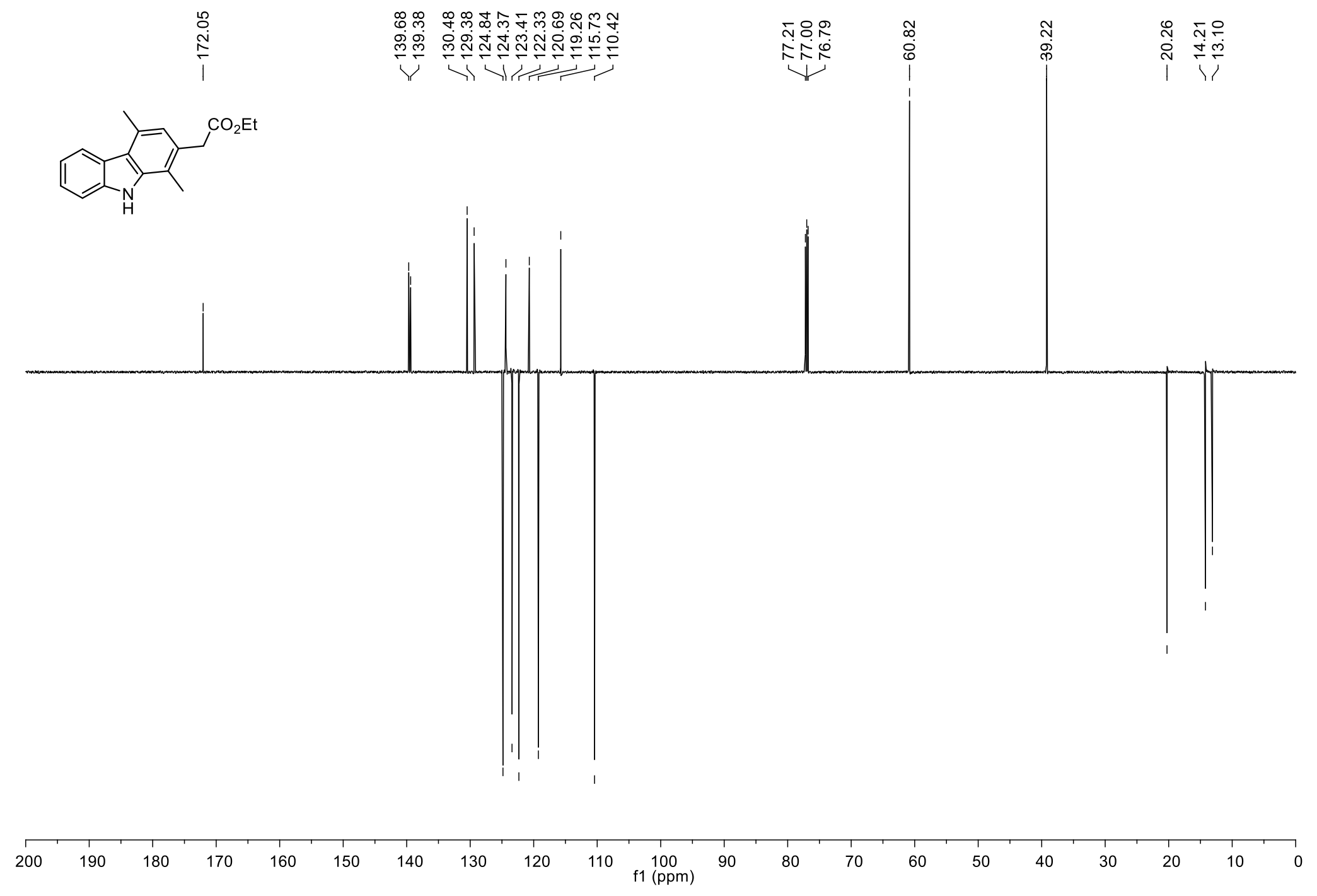

${ }^{13} \mathrm{C}$-NMR Spectrum of $15 \mathrm{~b}\left(150 \mathrm{MHz}, \mathrm{CDCl}_{3}\right.$ ) 


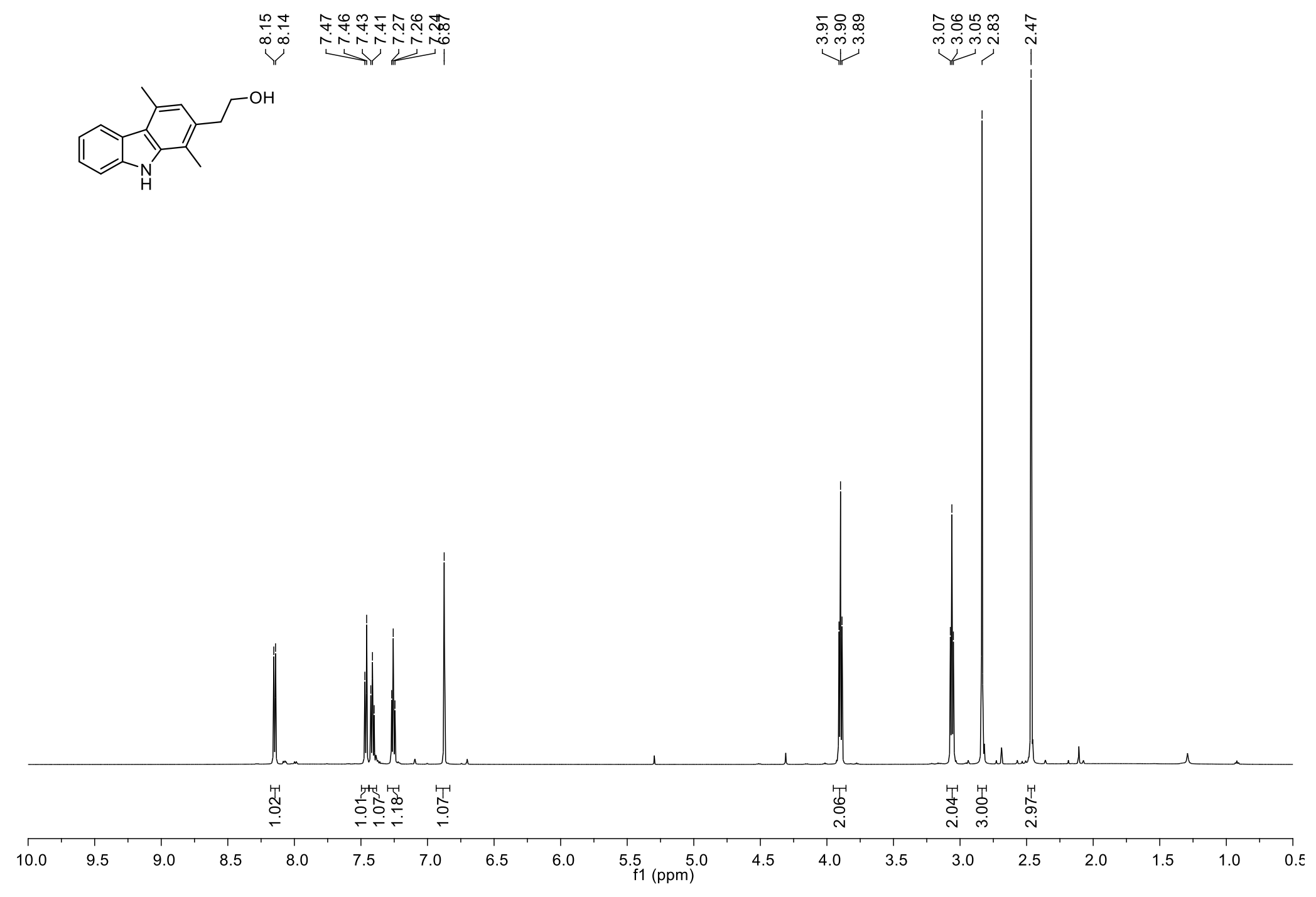

${ }^{1} \mathrm{H}-\mathrm{NMR}$ Spectrum of $21 \mathrm{~b}\left(600 \mathrm{MHz}, \mathrm{CDCl}_{3}\right)$ 


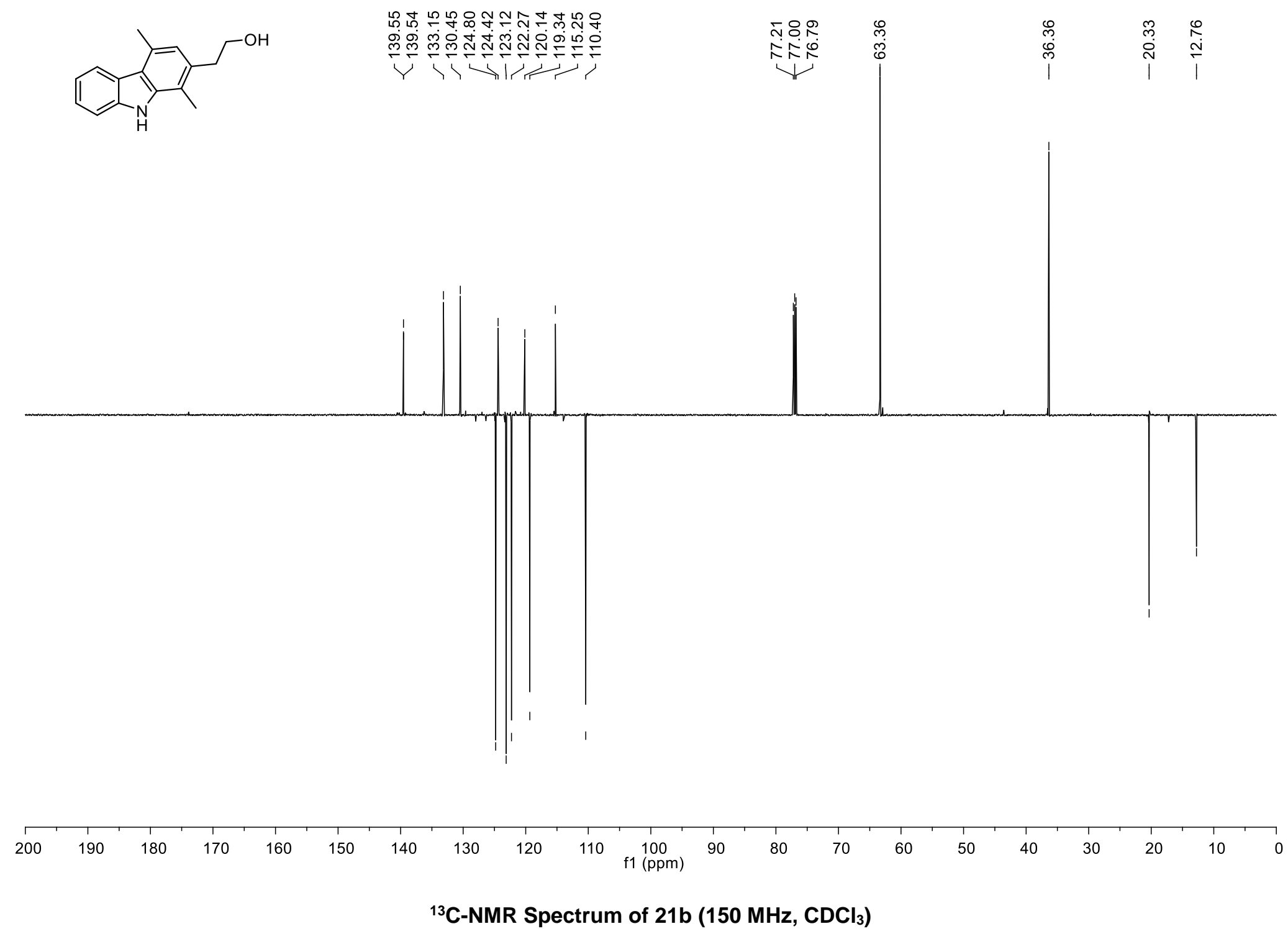




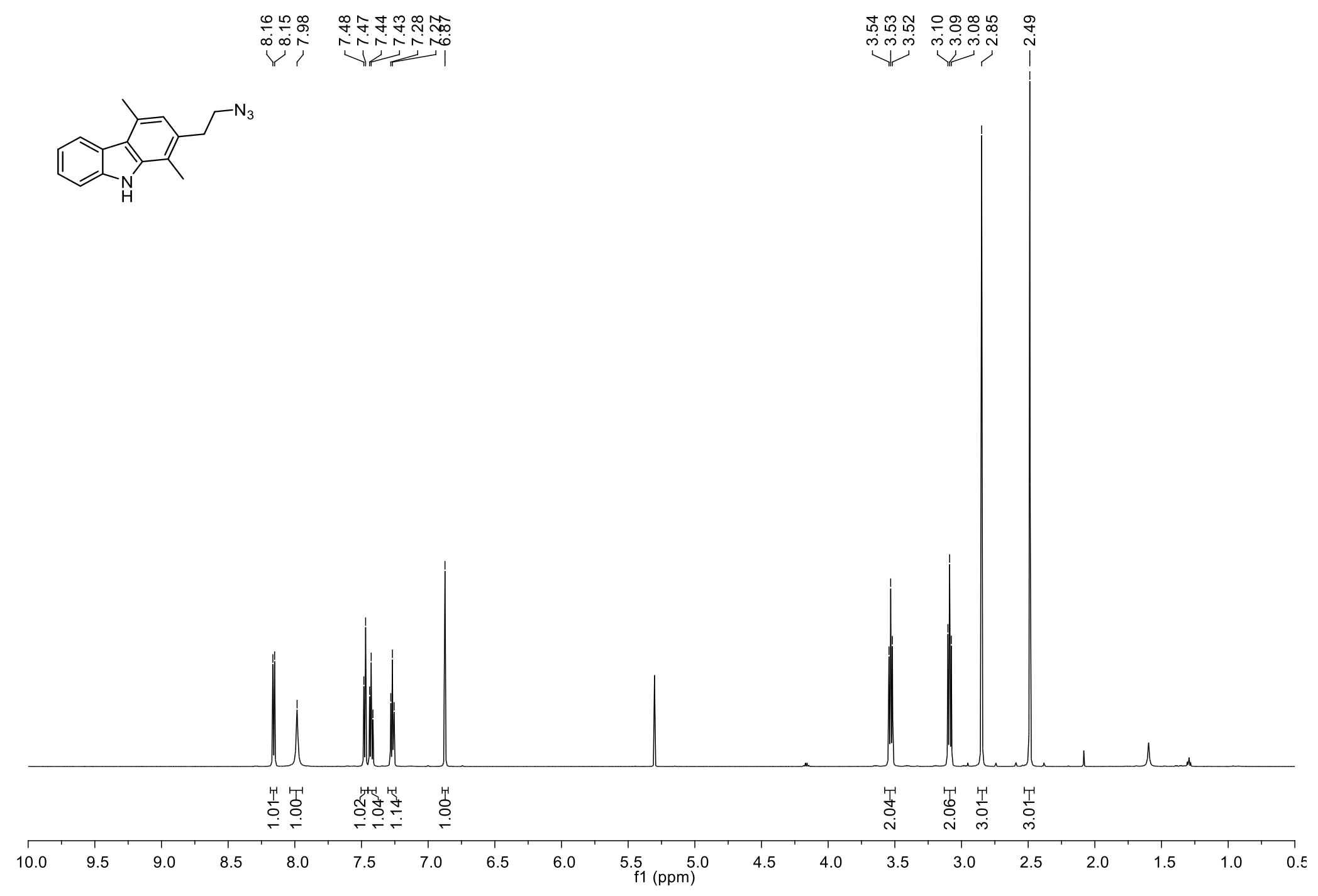

${ }^{1} \mathrm{H}-\mathrm{NMR}$ Spectrum of 23b (600 $\left.\mathrm{MHz}, \mathrm{CDCl}_{3}\right)$ 


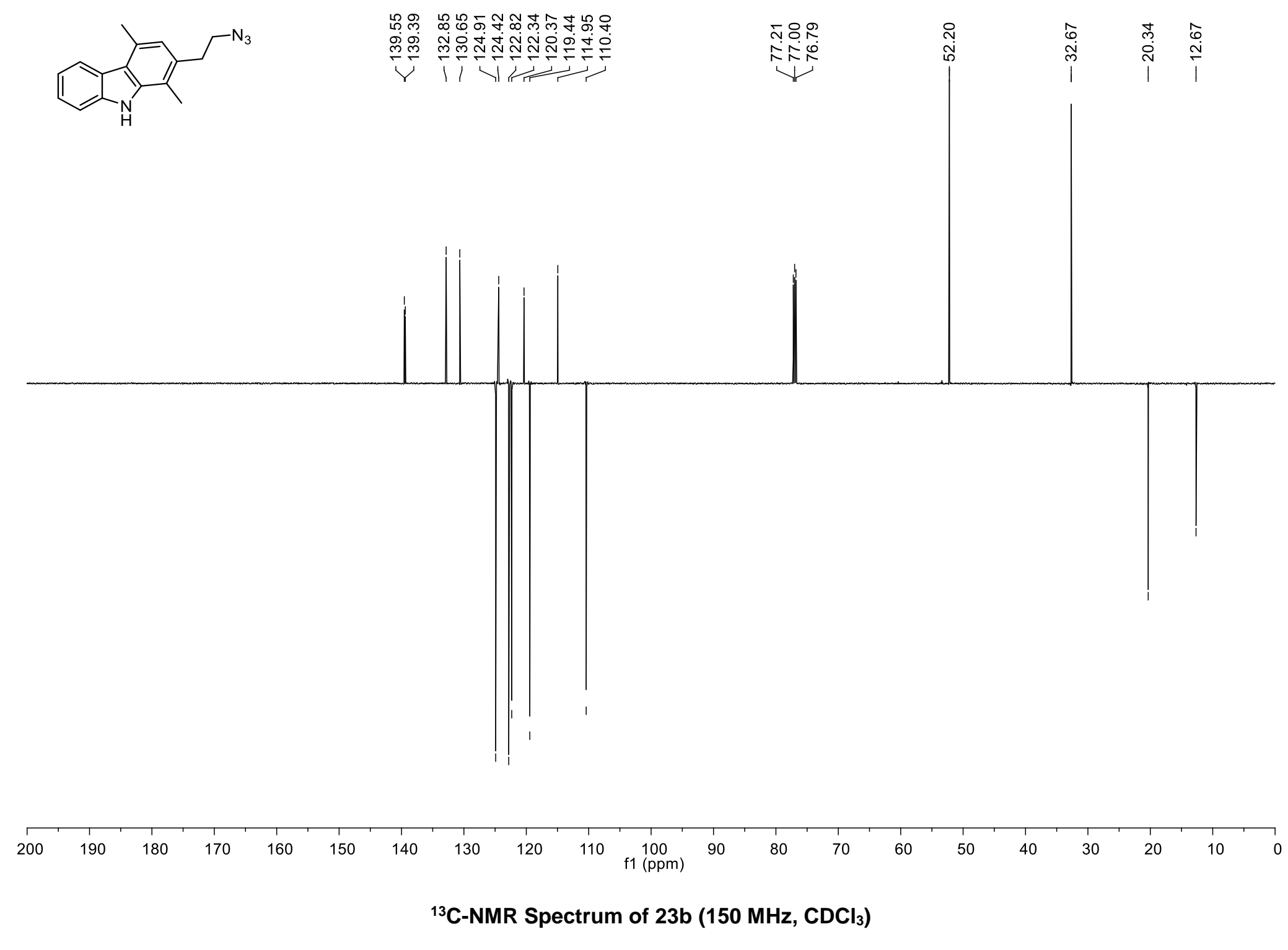




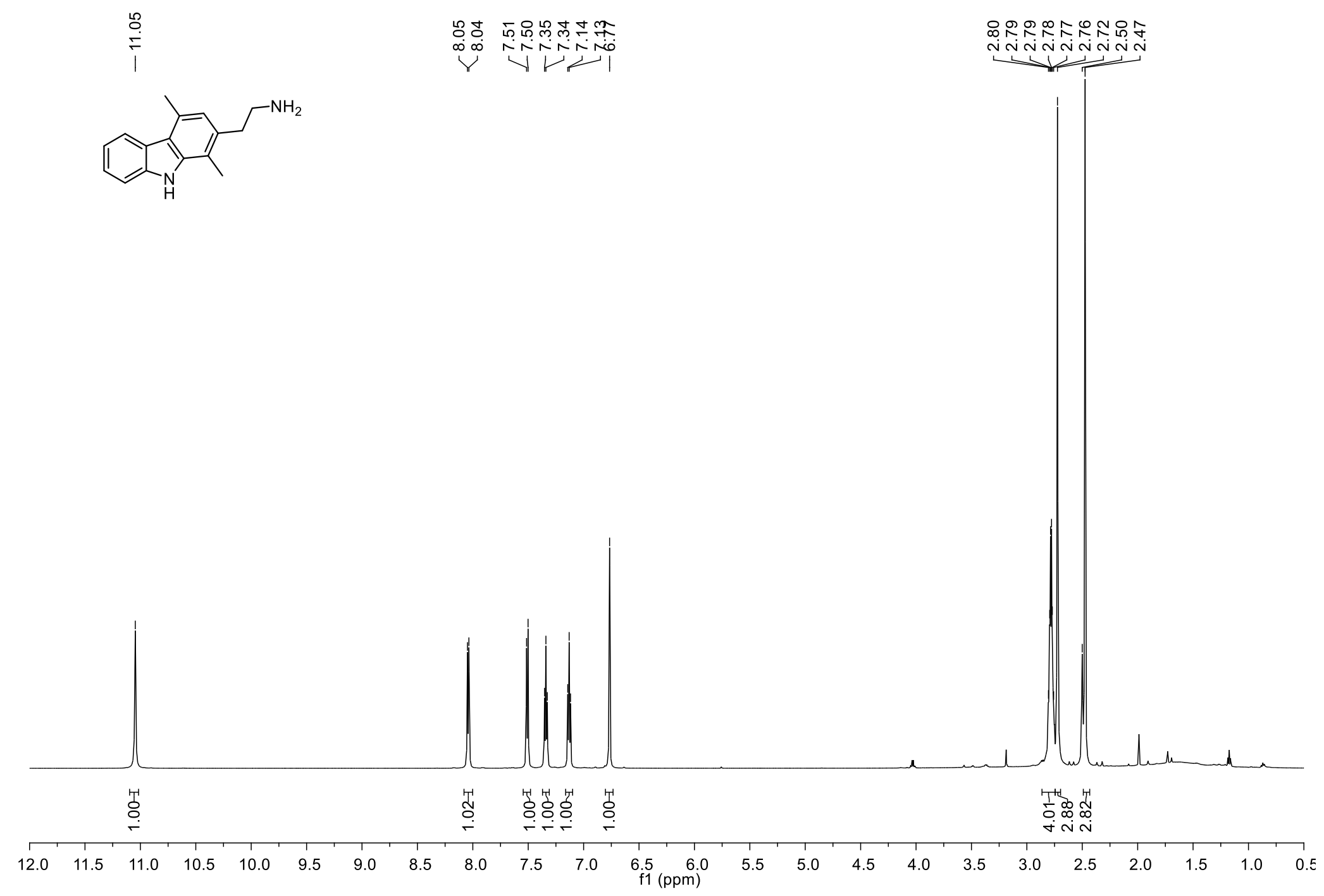

${ }^{1} \mathrm{H}-\mathrm{NMR}$ Spectrum of 24b (600 MHz, DMSO- $\left.d_{6}\right)$ 


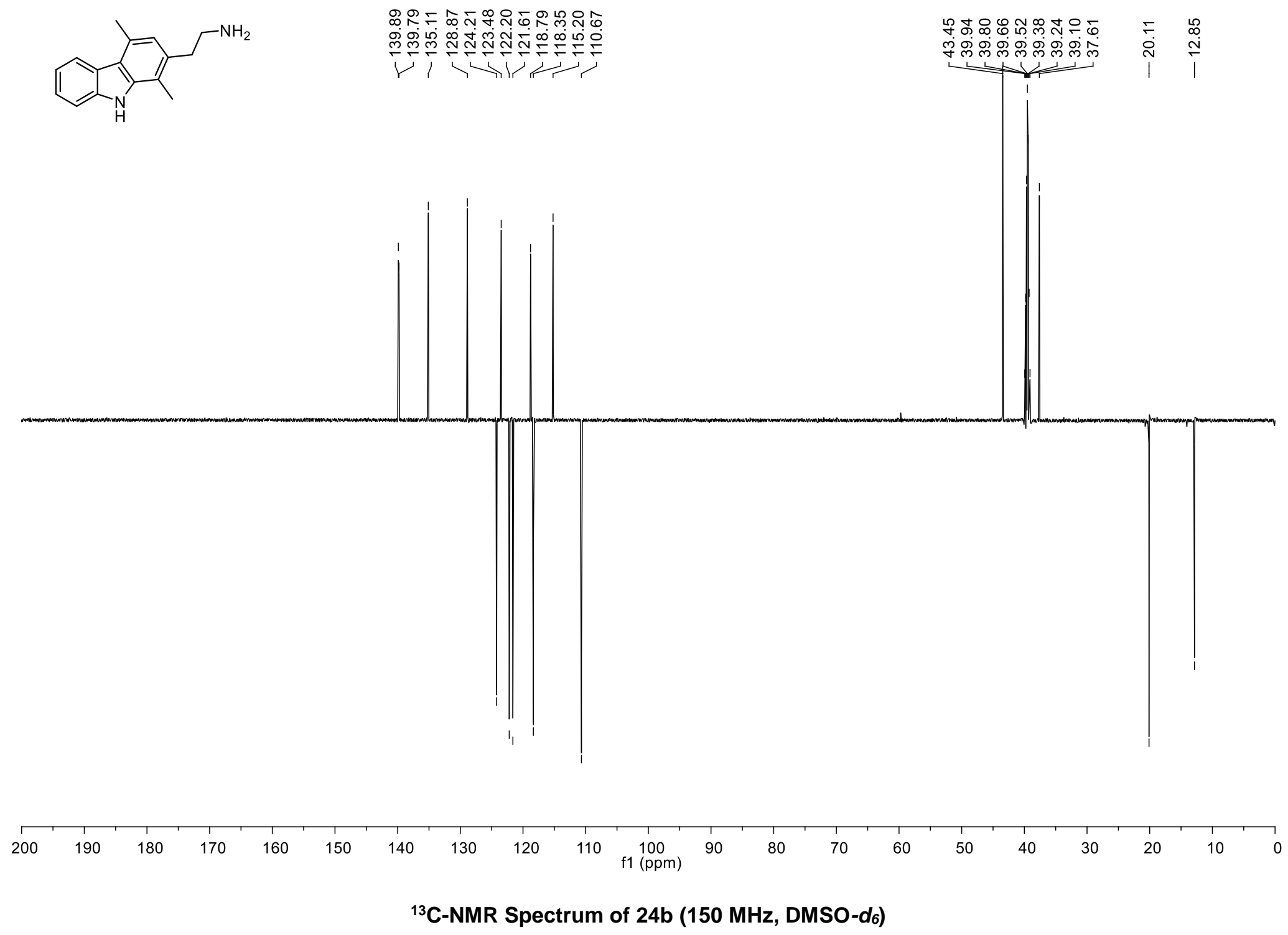




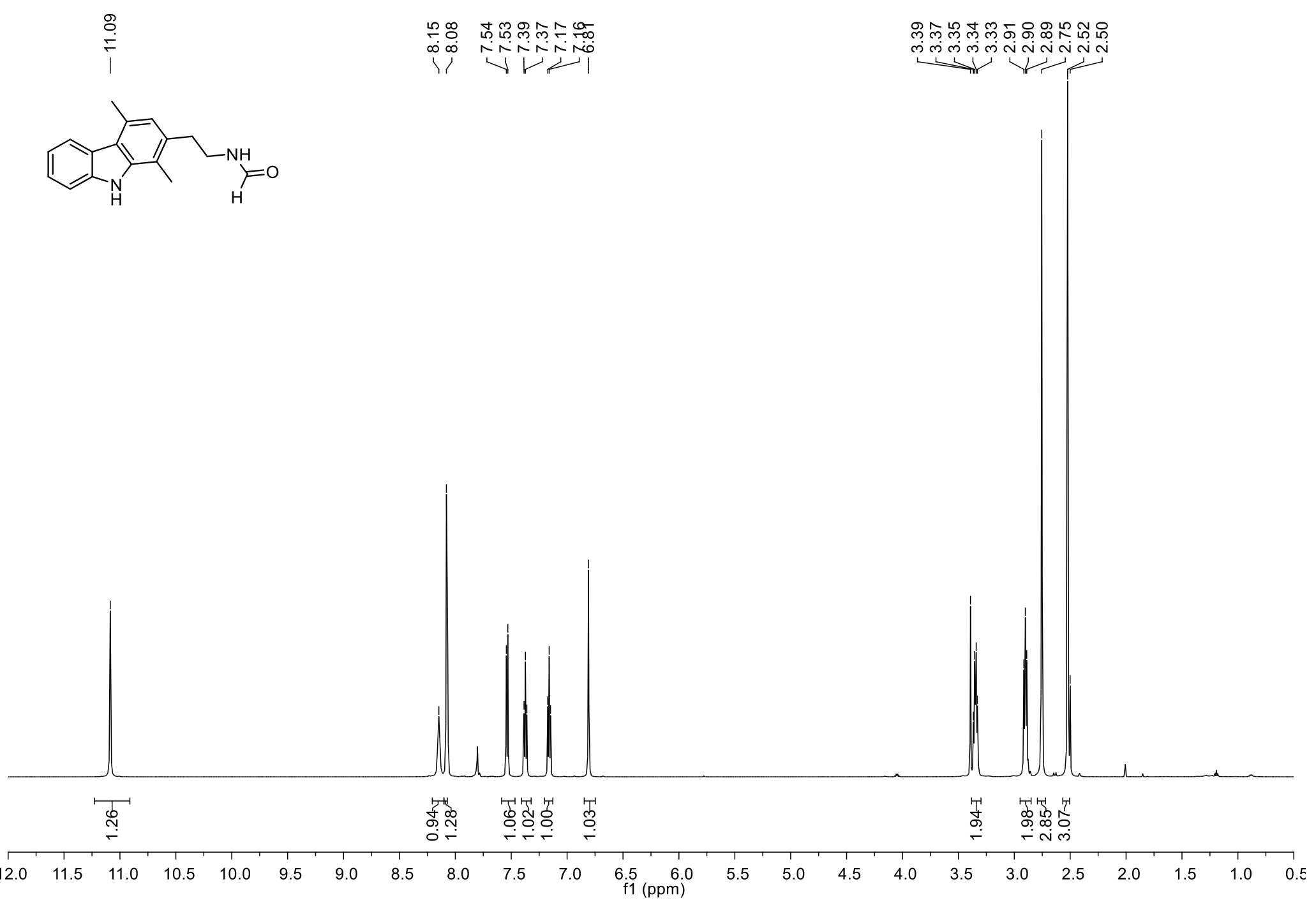

${ }^{1} \mathrm{H}-\mathrm{NMR}$ Spectrum of 27 (600 MHz, DMSO- $\left.d_{6}\right)$ 


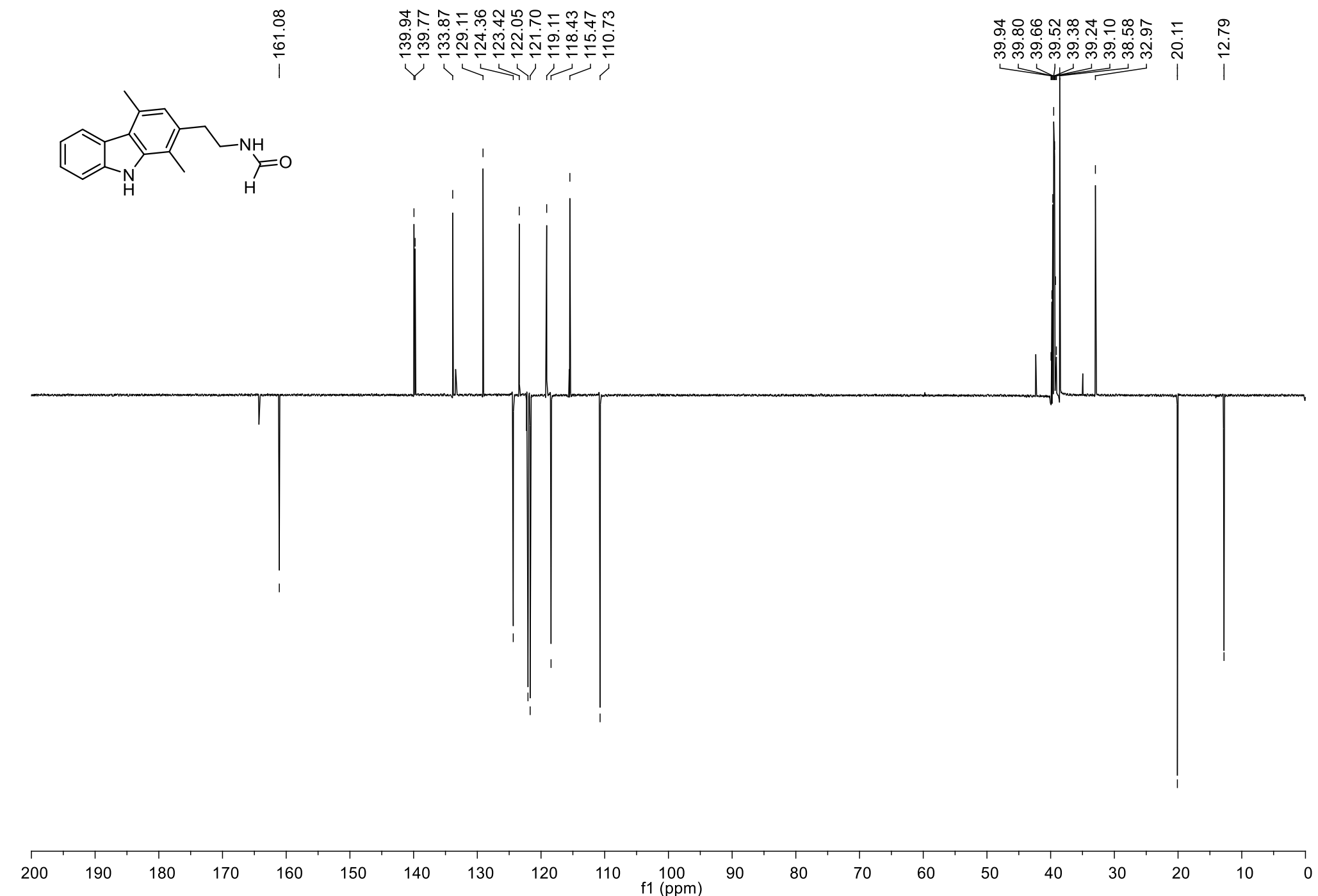

${ }^{13} \mathrm{C}-N M R$ Spectrum of 27 (150 MHz, DMSO-d $d_{6}$ ) 


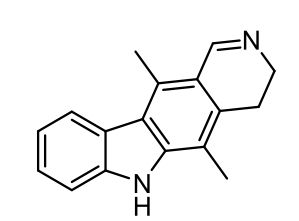

i

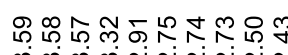

每
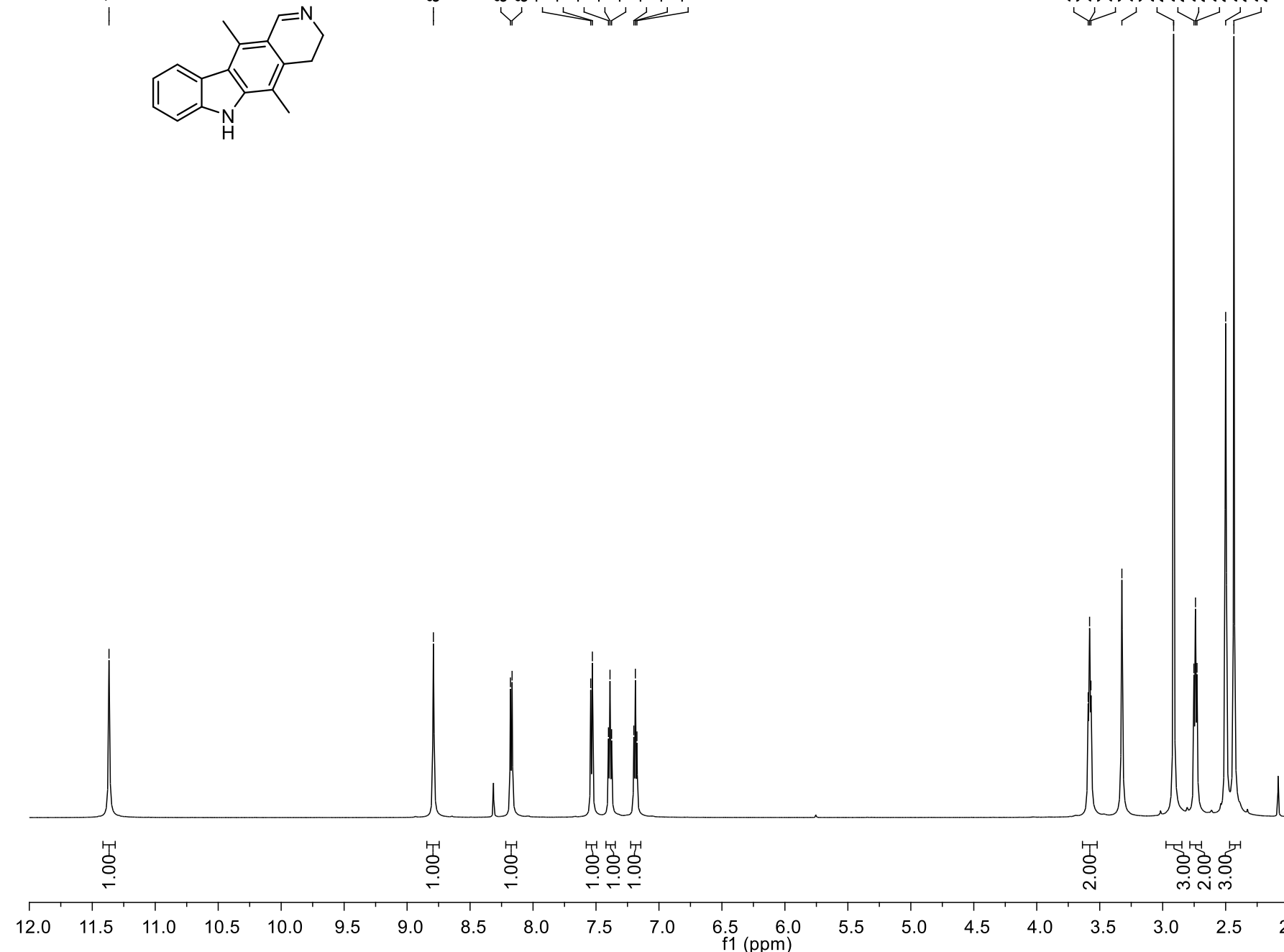

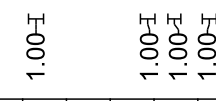

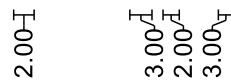

$\begin{array}{lllll}12.0 & 11.5 & 11.0 & 10.5 & 10.0\end{array}$

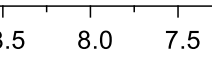

7.0

6.5
f1

${ }^{1} \mathrm{H}-\mathrm{NMR}$ Spectrum of 28 (600 MHz, DMSO-d $)^{\text {) }}$ 


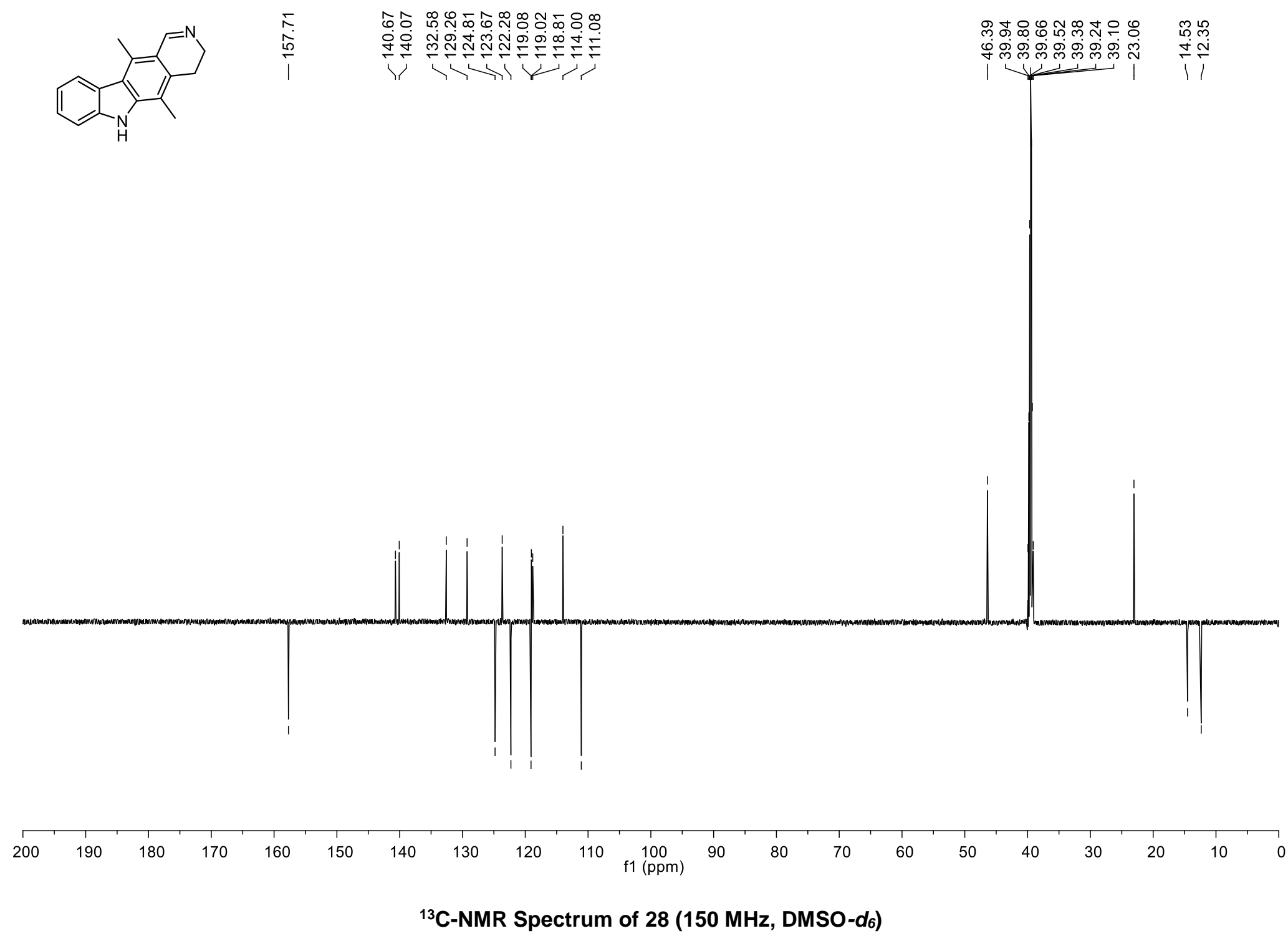




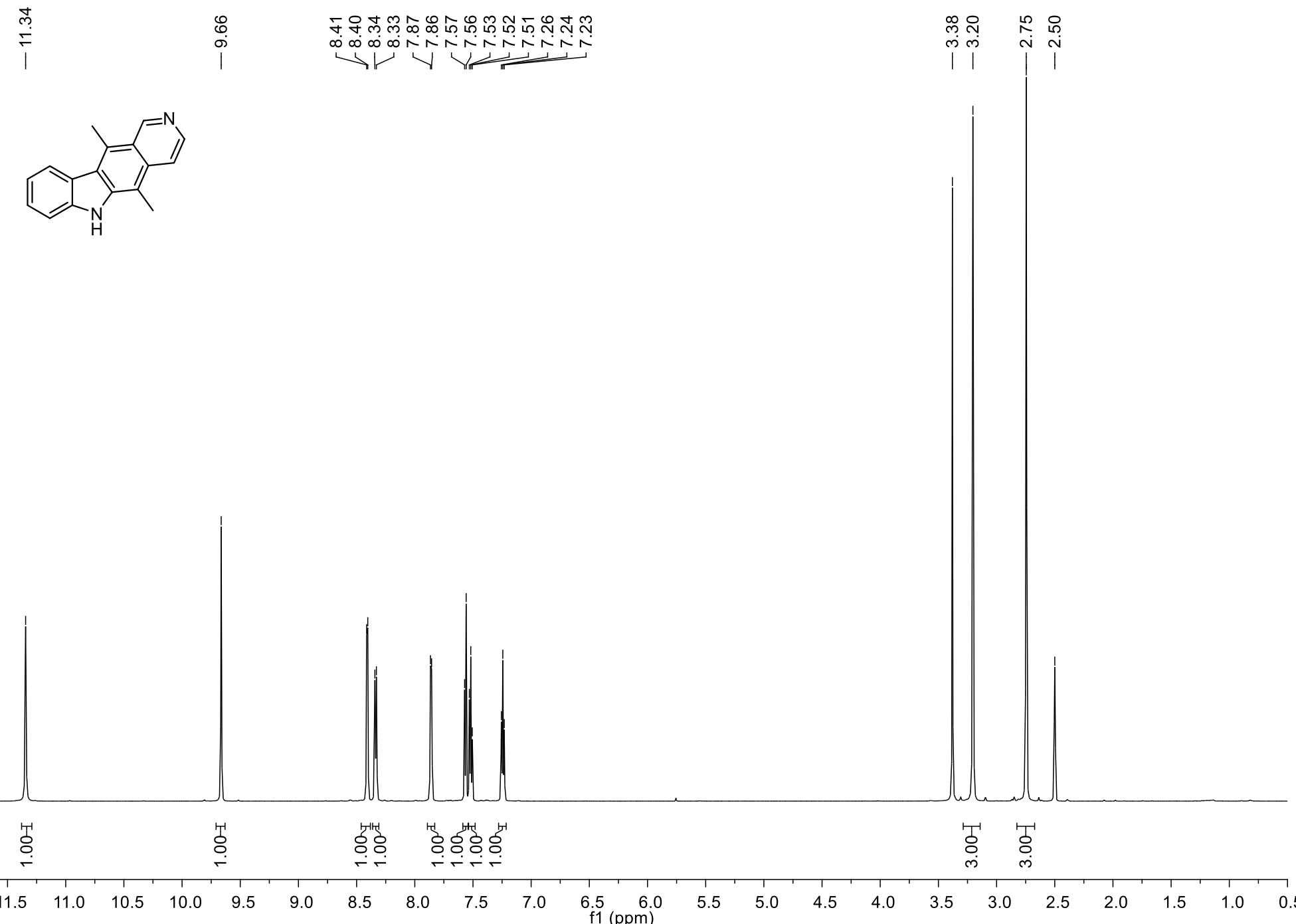

${ }^{1} \mathrm{H}-\mathrm{NMR}$ Spectrum of Ellipticine (600 MHz, DMSO-d $)_{6}$ 


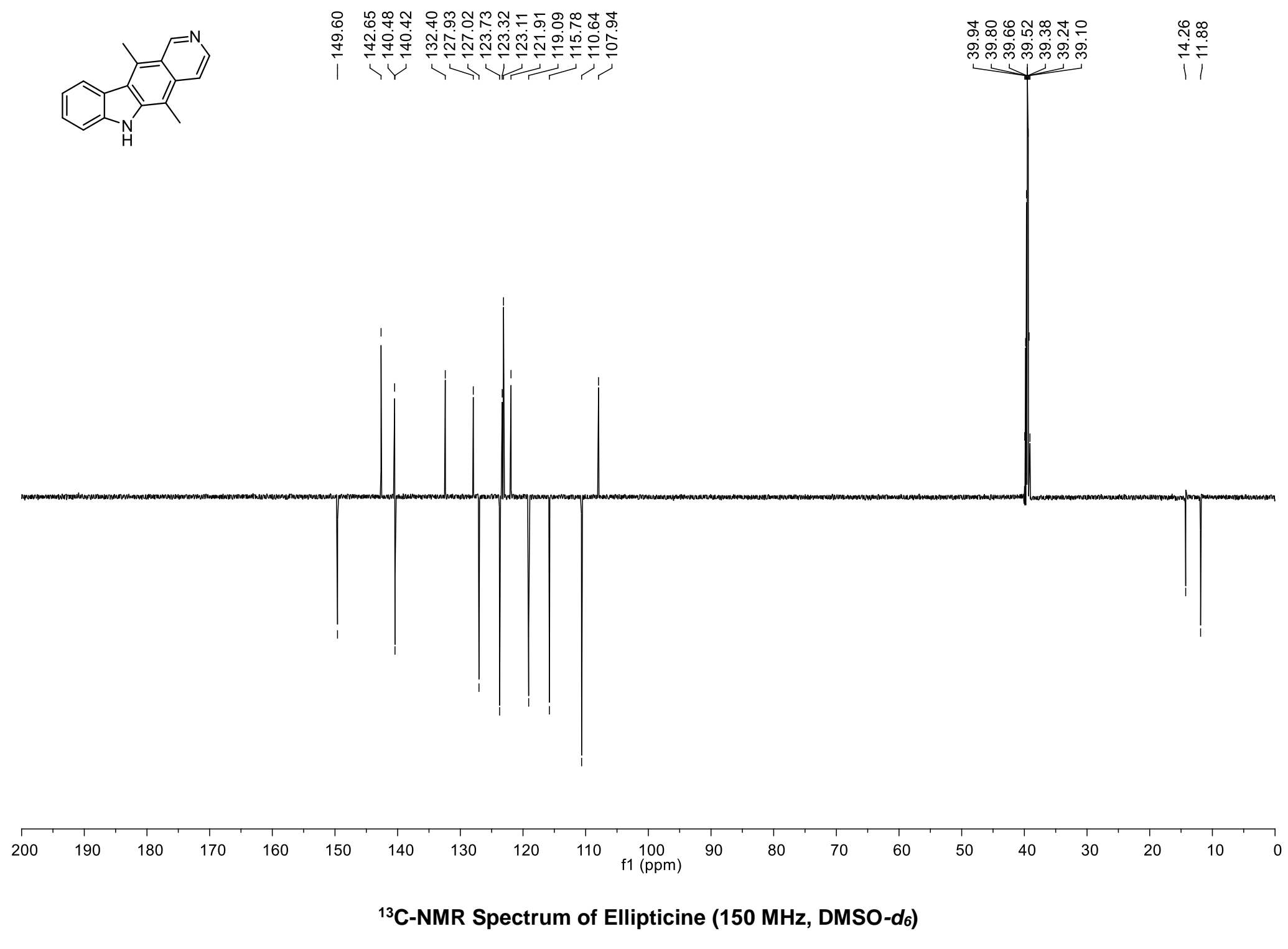



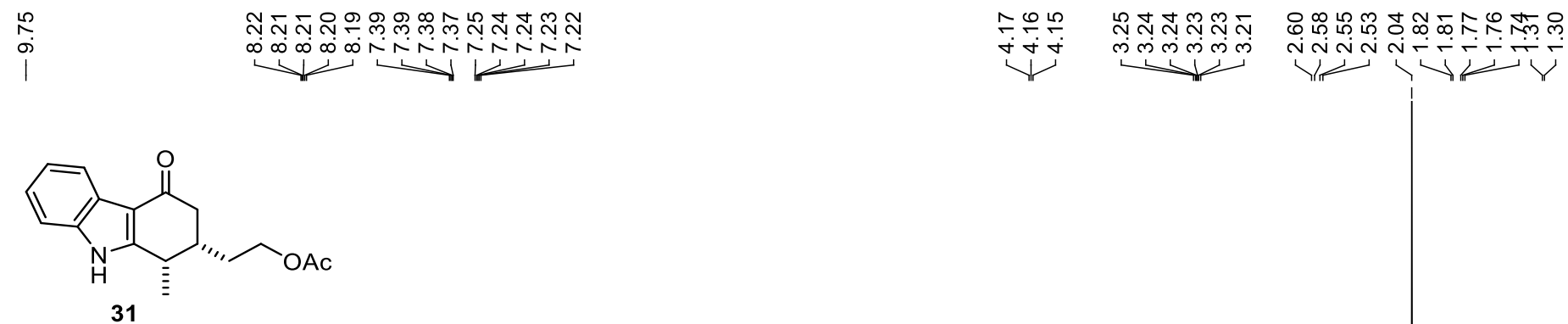

31

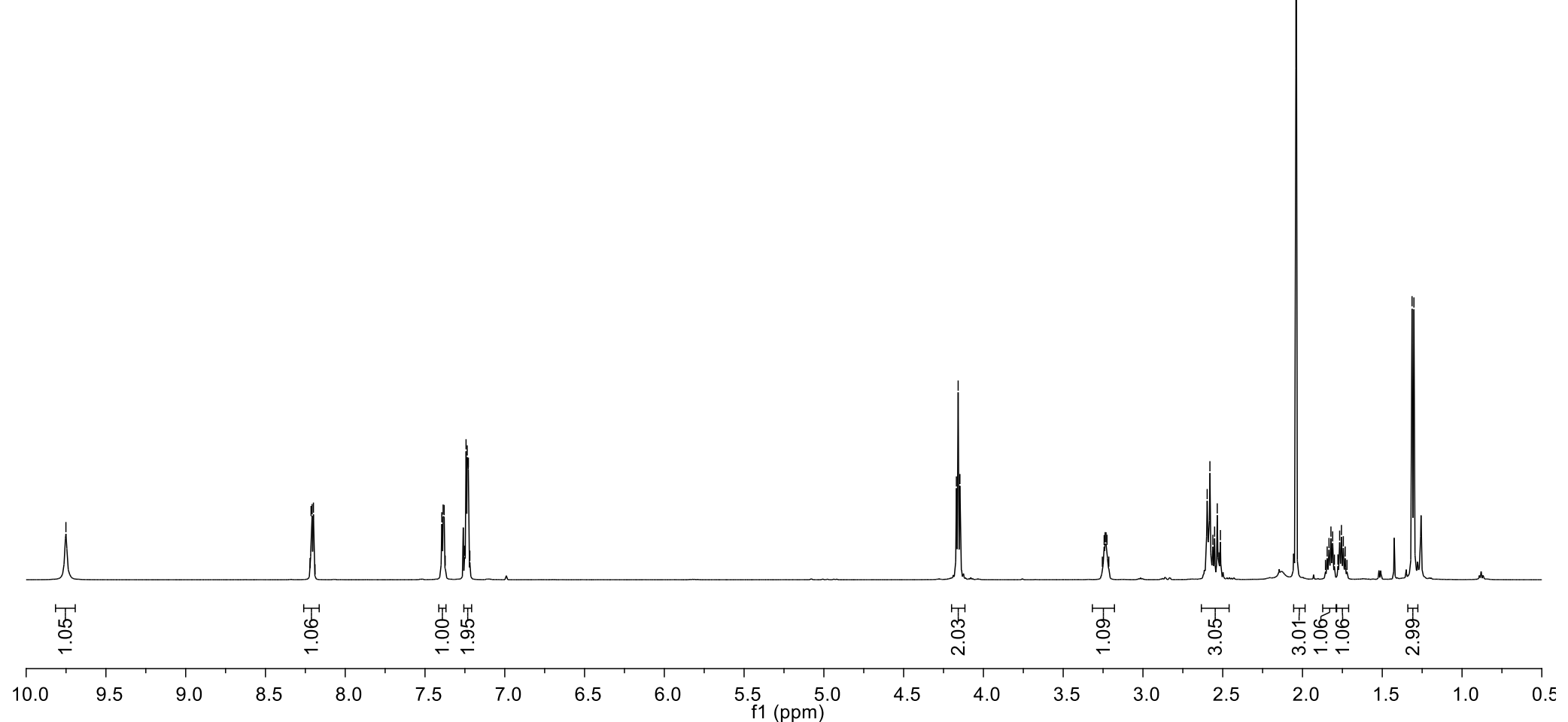

${ }^{1} \mathrm{H}-\mathrm{NMR}$ Spectrum of $31\left(600 \mathrm{MHz}, \mathrm{CDCl}_{3}\right)$ 


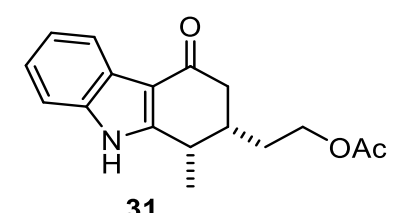

31

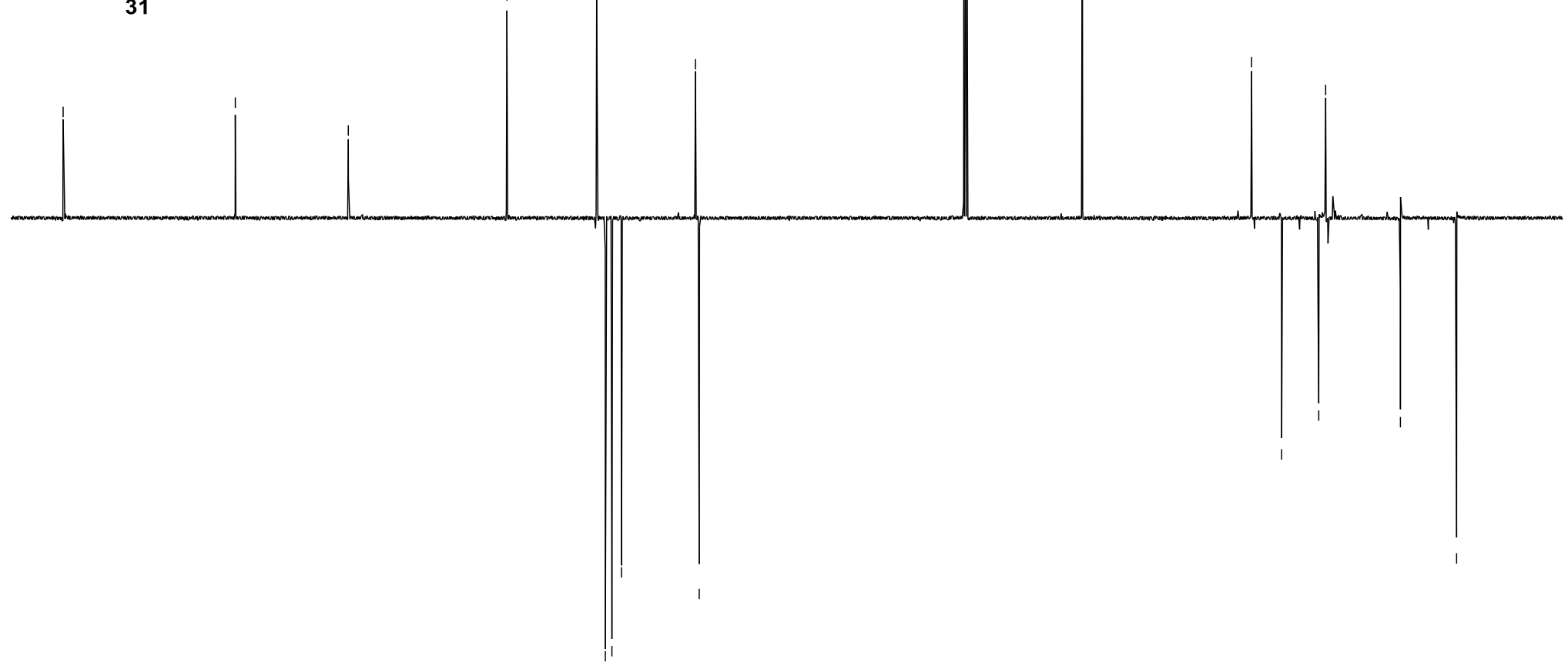

200

${ }^{13} \mathrm{C}$-NMR Spectrum of 31 (150 $\mathrm{MHz}, \mathrm{CDCl}_{3}$ ) 


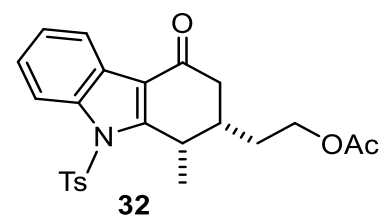

32

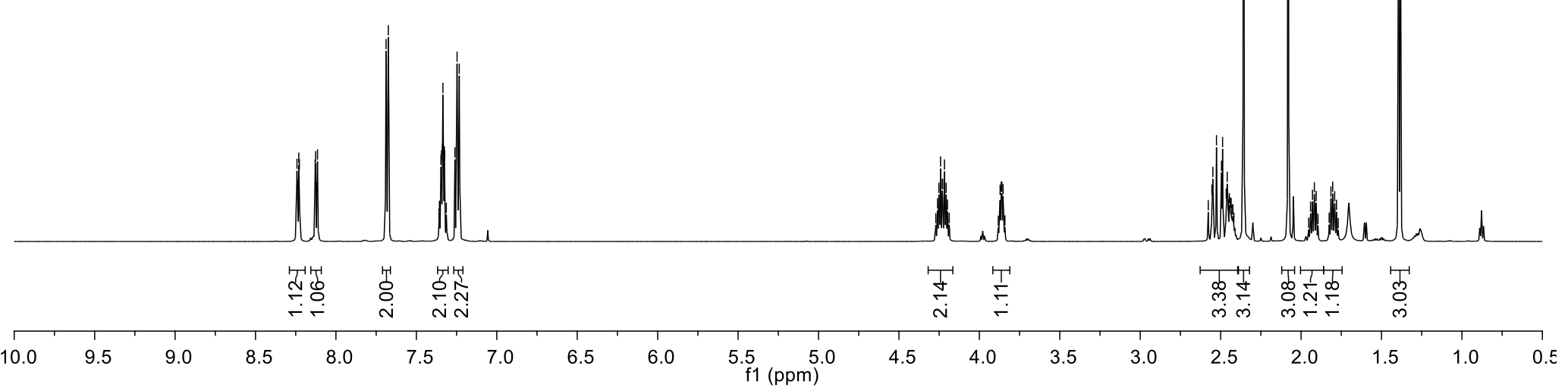

${ }^{1} \mathrm{H}-\mathrm{NMR}$ Spectrum of $32\left(600 \mathrm{MHz}, \mathrm{CDCl}_{3}\right)$ 


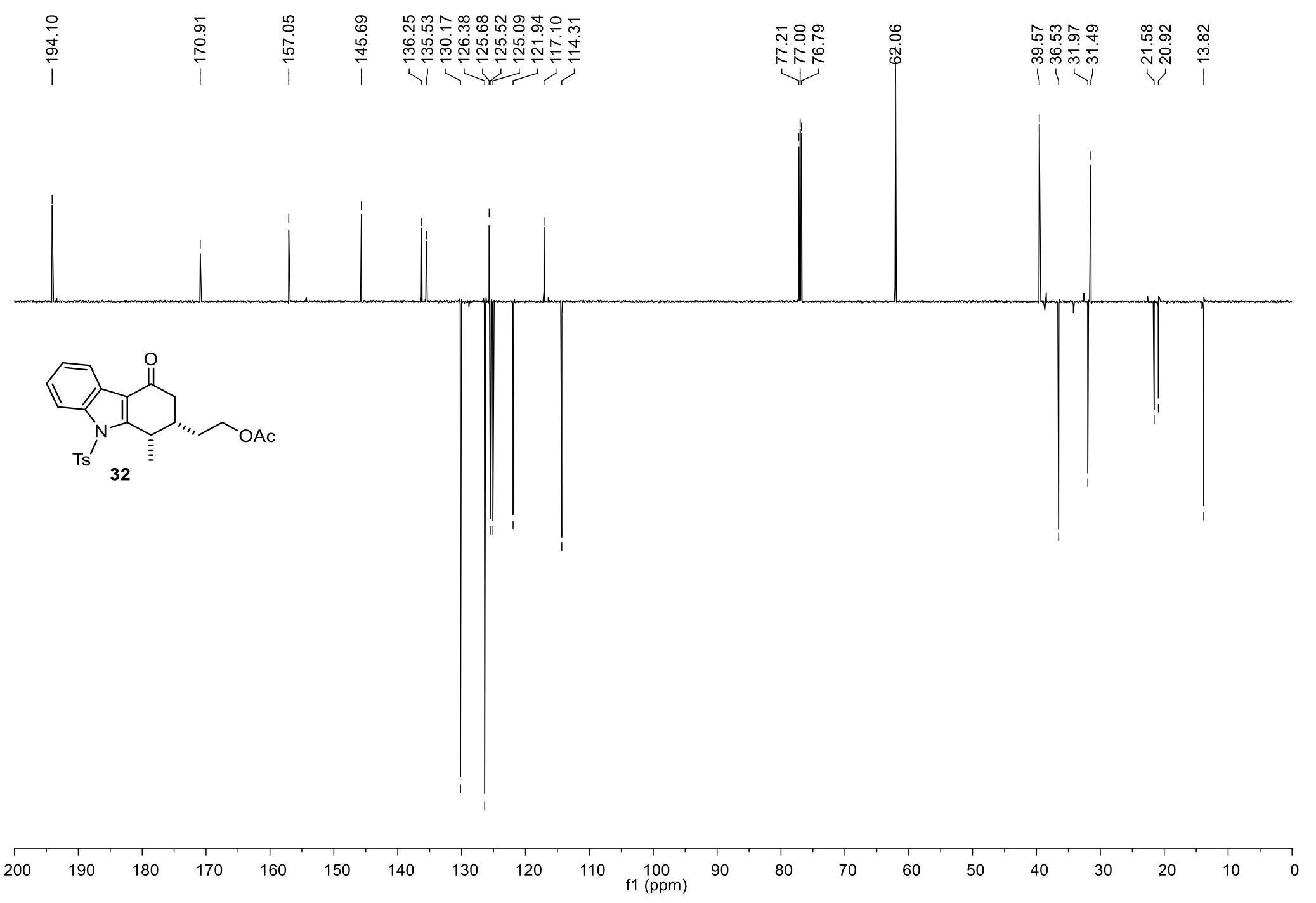

${ }^{13} \mathrm{C}$-NMR Spectrum of $32\left(150 \mathrm{MHz}, \mathrm{CDCl}_{3}\right.$ ) 


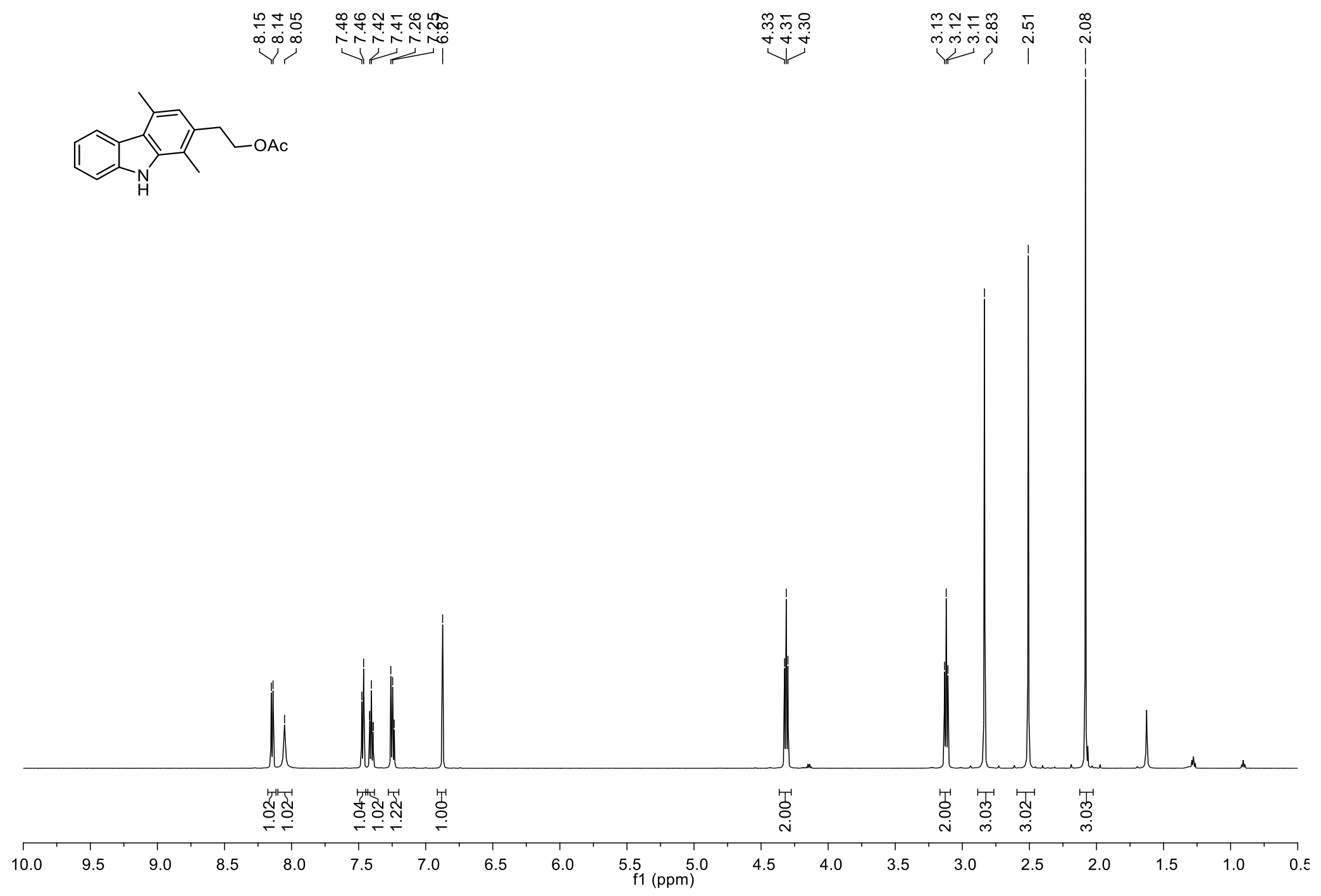

${ }^{1} \mathrm{H}-\mathrm{NMR}$ Spectrum of $35\left(600 \mathrm{MHz}, \mathrm{CDCl}_{3}\right)$ 

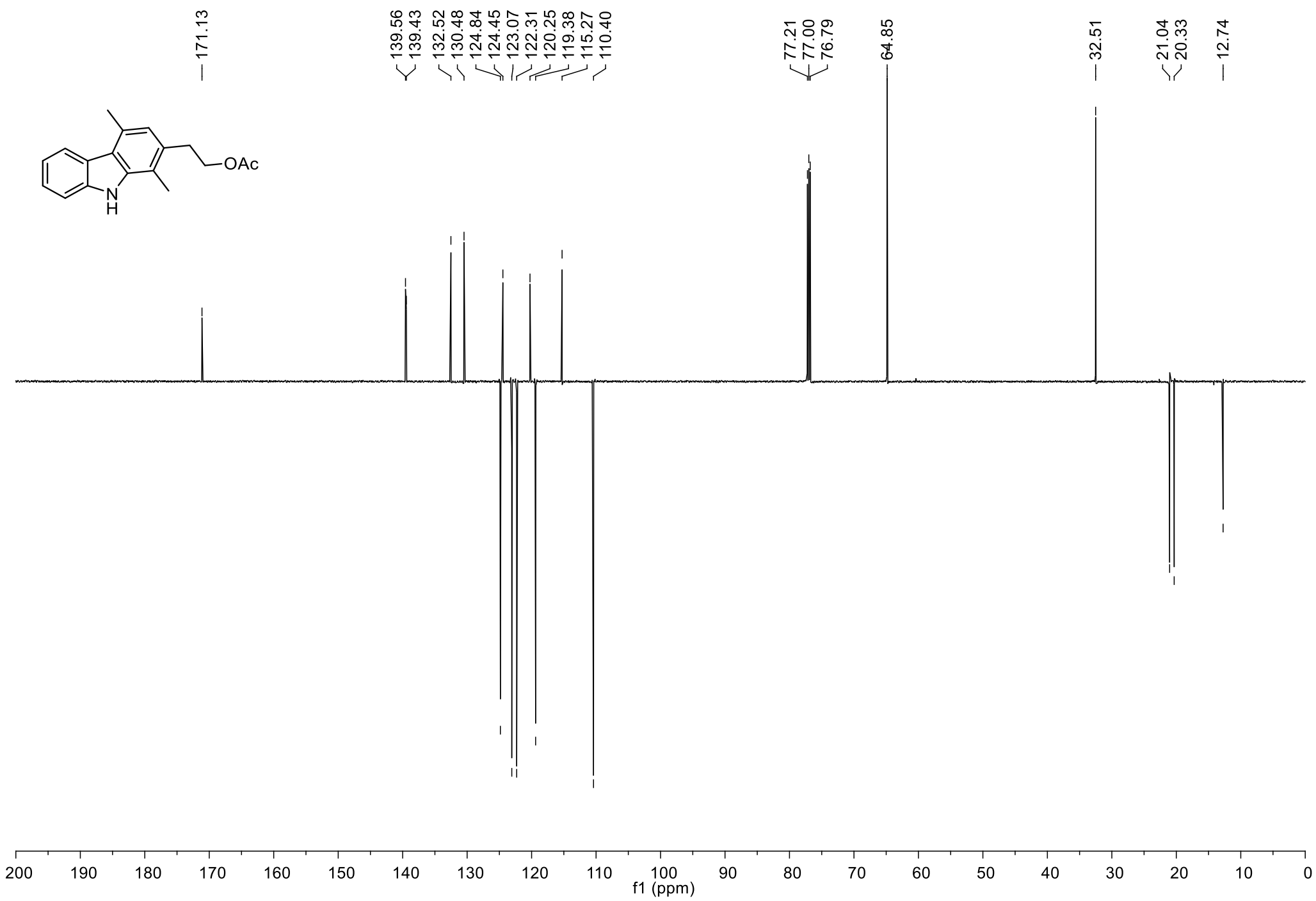

${ }^{13} \mathrm{C}-\mathrm{NMR}$ Spectrum of $35\left(150 \mathrm{MHz}, \mathrm{CDCl}_{3}\right)$

S105 